\title{
Synthesis, Characterization and Reactivities of Molybdenum and Tungsten PONOP Pincer Complexes
}

Ruth Castro-Rodrigo ${ }^{\ddagger}$, Sumit Chakraborty ${ }^{\ddagger}$, Lloyd Munjanja ${ }^{\ddagger}$, William W. Brennessel, and William D. Jones*

Department of Chemistry, University of Rochester, Rochester, New York 14627, United States.

Table of Contents:

\begin{tabular}{|l|l|}
\hline Characterization data for the Complexes 2a $-\mathbf{d}, \mathbf{3 a}-\mathbf{d}$ (NMR and IR) & $\mathrm{S} 1-\mathrm{S} 29$ \\
\hline${ }^{13} \mathrm{CO}$ incorporation experiments & $\mathrm{S} 30$ \\
\hline NMR spectra for the 1-hexene isomerization experiments & $\mathrm{S} 31-\mathrm{S} 32$ \\
\hline NMR data, kinetic plot, and GC data for the isomerization of allylbenzene & $\mathrm{S} 33-\mathrm{S} 34$ \\
\hline NMR and GC data for the isomerization of 1-octene & $\mathrm{S} 35-\mathrm{S} 36$ \\
\hline Variable Temperature ${ }^{1}$ H NMR of complex 3d & $\mathrm{S} 37$ \\
\hline Simulation of hydride resonance for complex 3b. & $\mathrm{S} 38$ \\
\hline Eyring plot for hydride fluxionality in complex 3b. & $\mathrm{S} 39$ \\
\hline Crystal Data and Refinement Parameters for 2a-d. & $\mathrm{S} 40$ \\
\hline Selected bond lengths $(\AA)$ and angles (deg) for 2a-d. & $\mathrm{S} 41$ \\
\hline X-ray Characterization data for the Complexes 2a - d & $\mathrm{S} 42-\mathrm{S} 91$ \\
\hline
\end{tabular}


Characterization for Complex 2a [( $\mathrm{BuPONOP}) \mathrm{Mo}(\mathrm{CO})_{3}$

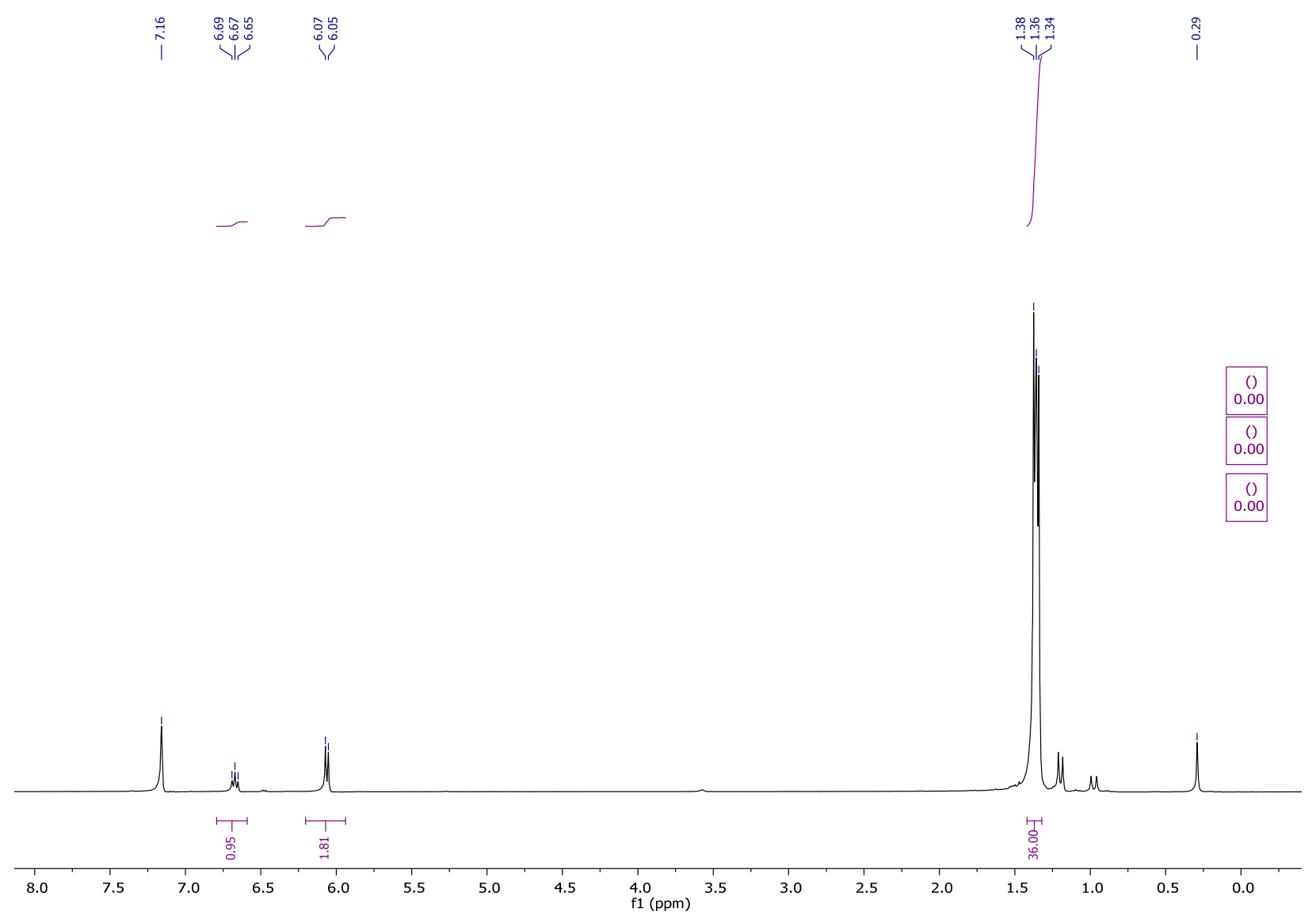

Figure $\mathbf{S 1}-{ }^{1} \mathrm{H}$ NMR of $\mathbf{2 a} \mathrm{C}_{6} \mathrm{D}_{6}$ at $\mathrm{RT}$ 

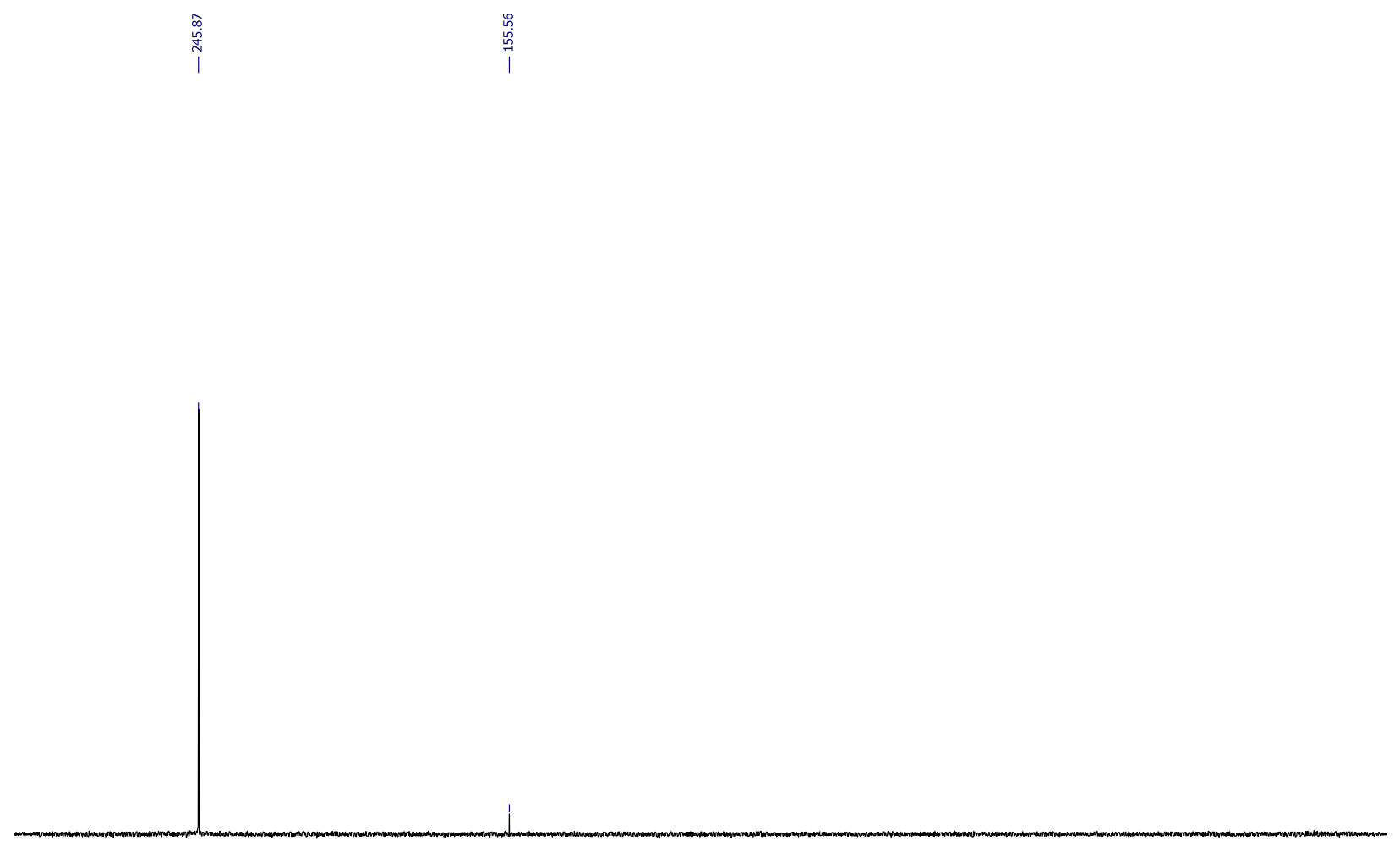

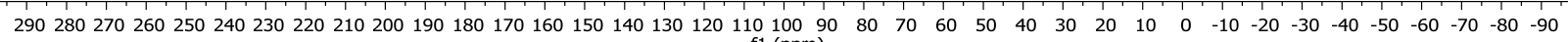
f1 (ppm)

Figure $\mathbf{S 2}-{ }^{31} \mathrm{P}\left\{{ }^{1} \mathrm{H}\right\} \mathrm{NMR}^{\mathrm{C}} \mathrm{C}_{6} \mathrm{D}_{6}$ at $\mathrm{RT}$ for $\mathbf{2 a}$ 


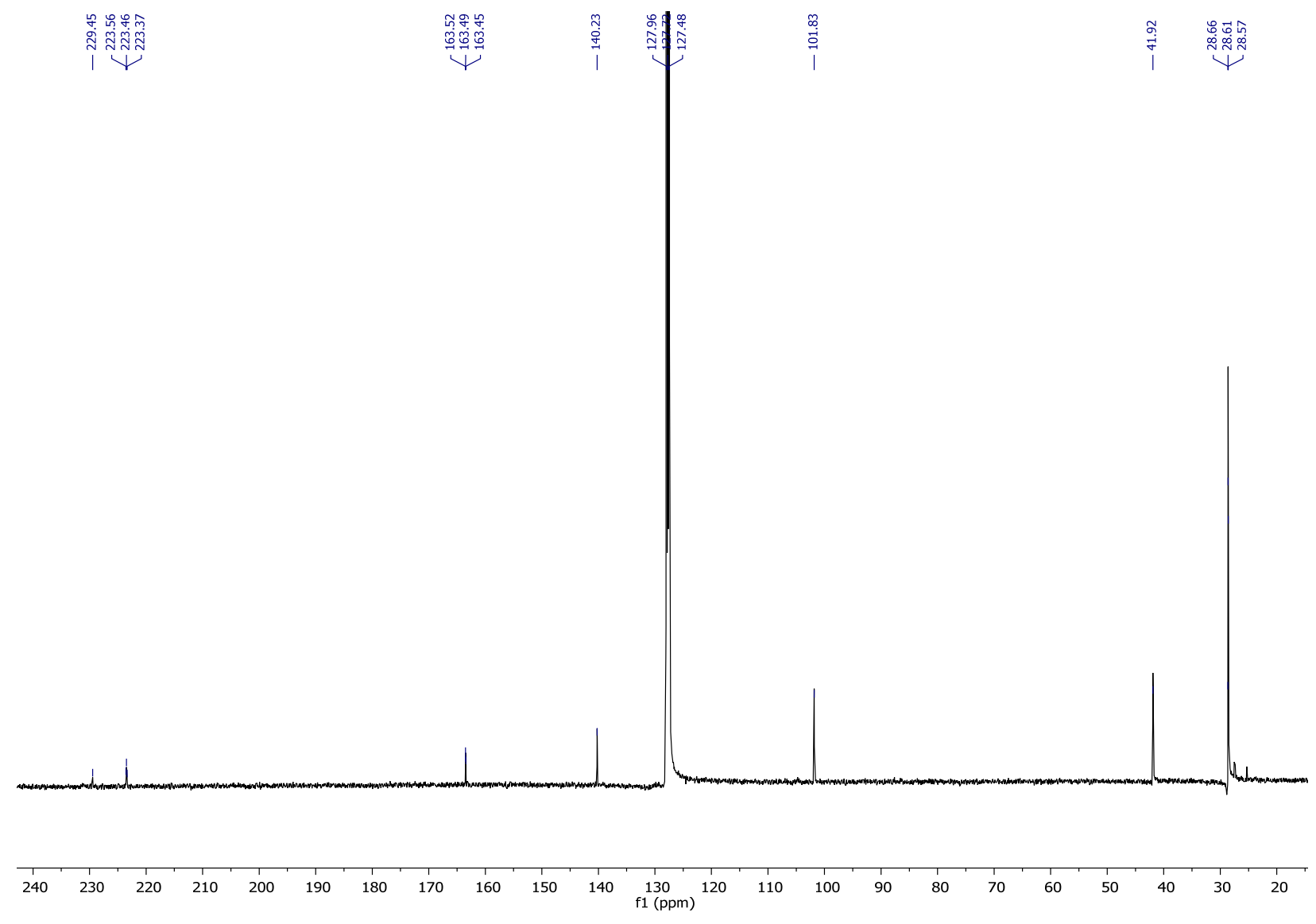

Figure $\mathbf{S 3}-{ }^{13} \mathrm{C}\left\{{ }^{1} \mathrm{H}\right\} \quad \mathrm{NMR}$ of $\mathbf{2} \mathbf{a} \mathrm{C}_{6} \mathrm{D}_{6}$ at $\mathrm{RT}$ 
Characterization for Complex $\mathbf{2 b}\left[{ }^{\mathrm{i}} \mathrm{PrPONOP}\right) \mathrm{Mo}(\mathrm{CO})_{3}$
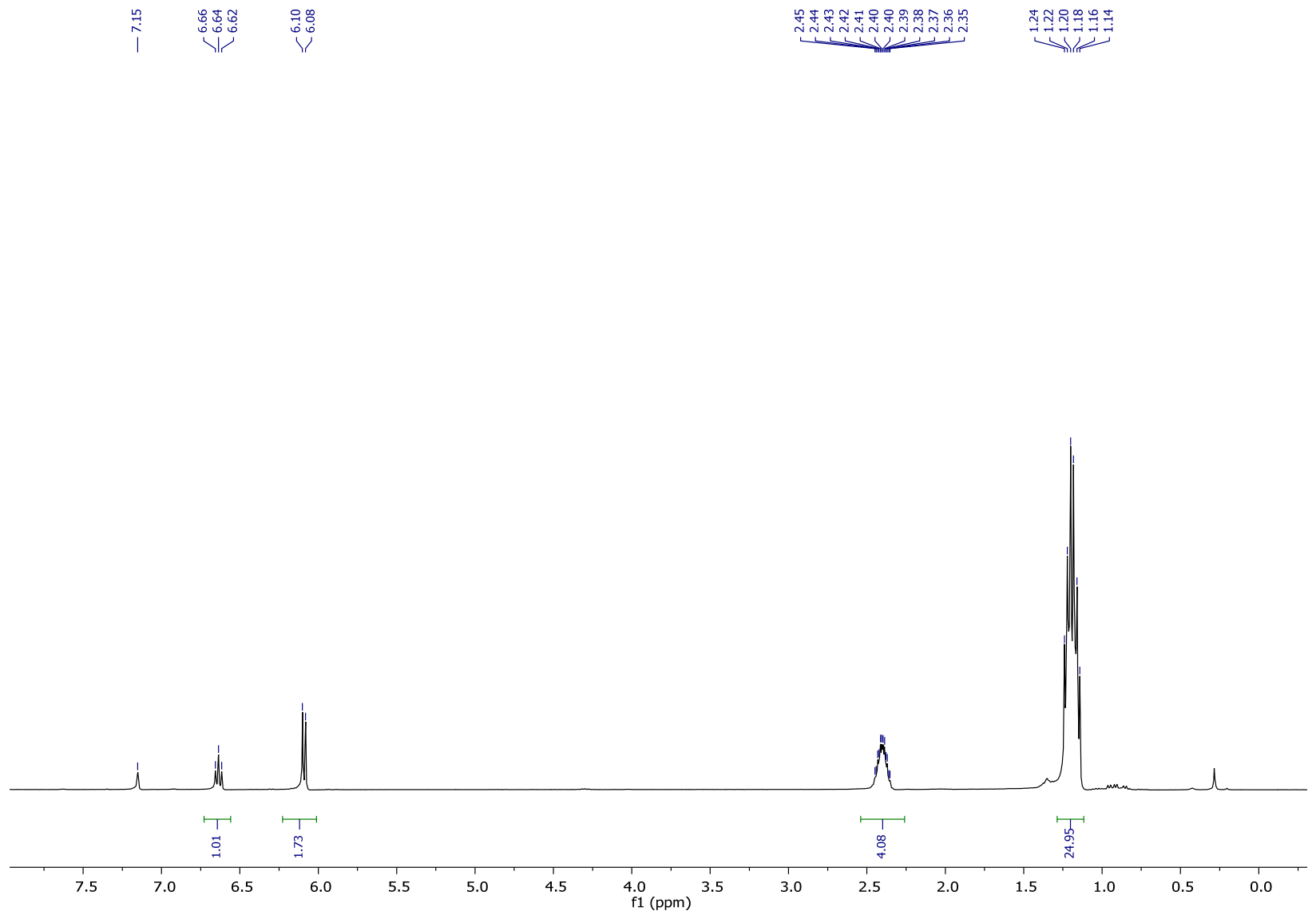

Figure $\mathbf{S 4}-{ }^{1} \mathrm{H}$ NMR of $\mathbf{2 b} \mathrm{C}_{6} \mathrm{D}_{6}$ at $\mathrm{RT}$ 


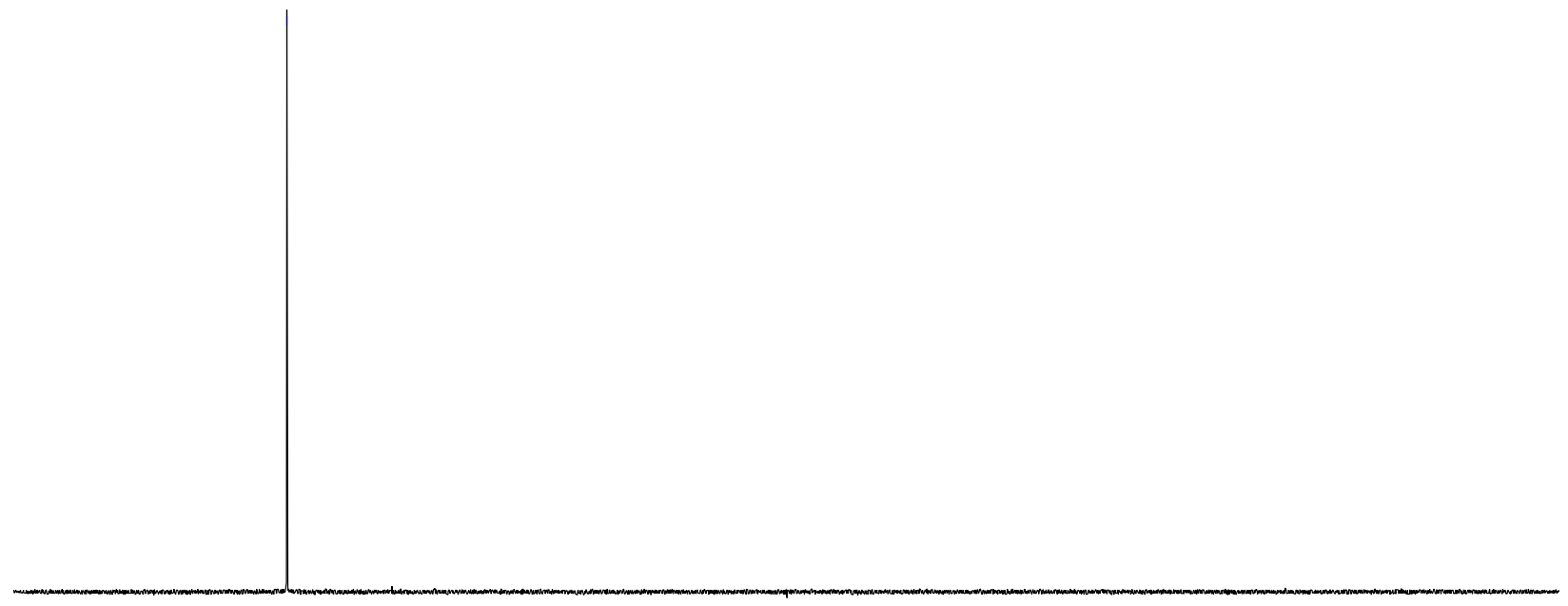

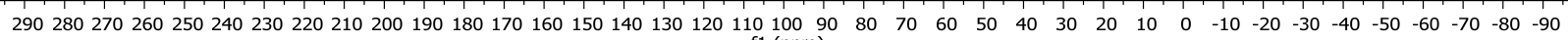
$\mathrm{f} 1(\mathrm{ppm})$

Figure S5 $-{ }^{31} \mathrm{P}\left\{{ }^{1} \mathrm{H}\right\} \mathrm{NMR} \mathrm{C}_{6} \mathrm{D}_{6}$ at $\mathrm{RT}$ for $\mathbf{2 b}$ 


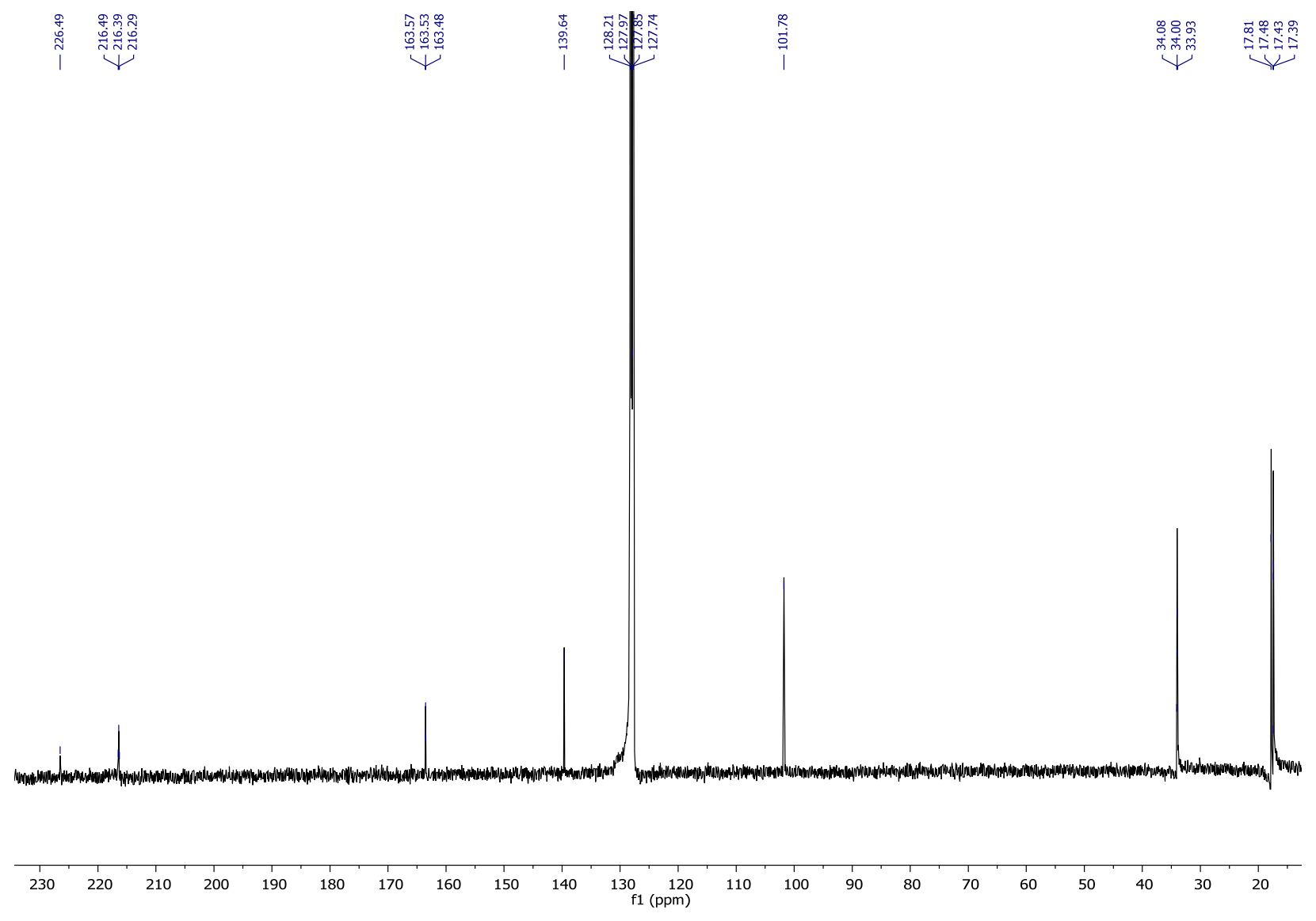

Figure S6 $-{ }^{13} \mathrm{C}\left\{{ }^{1} \mathrm{H}\right\} \quad \mathrm{NMR}$ of $\mathbf{2 b} \mathrm{C}_{6} \mathrm{D}_{6}$ at RT 
Characterization for Complex $\mathbf{2 c}\left[\left({ }^{\mathrm{t}} \mathrm{BuPONOP}\right) \mathrm{W}(\mathrm{CO})_{3}\right.$

$1 H$ NMR in $C_{6} D_{6}$

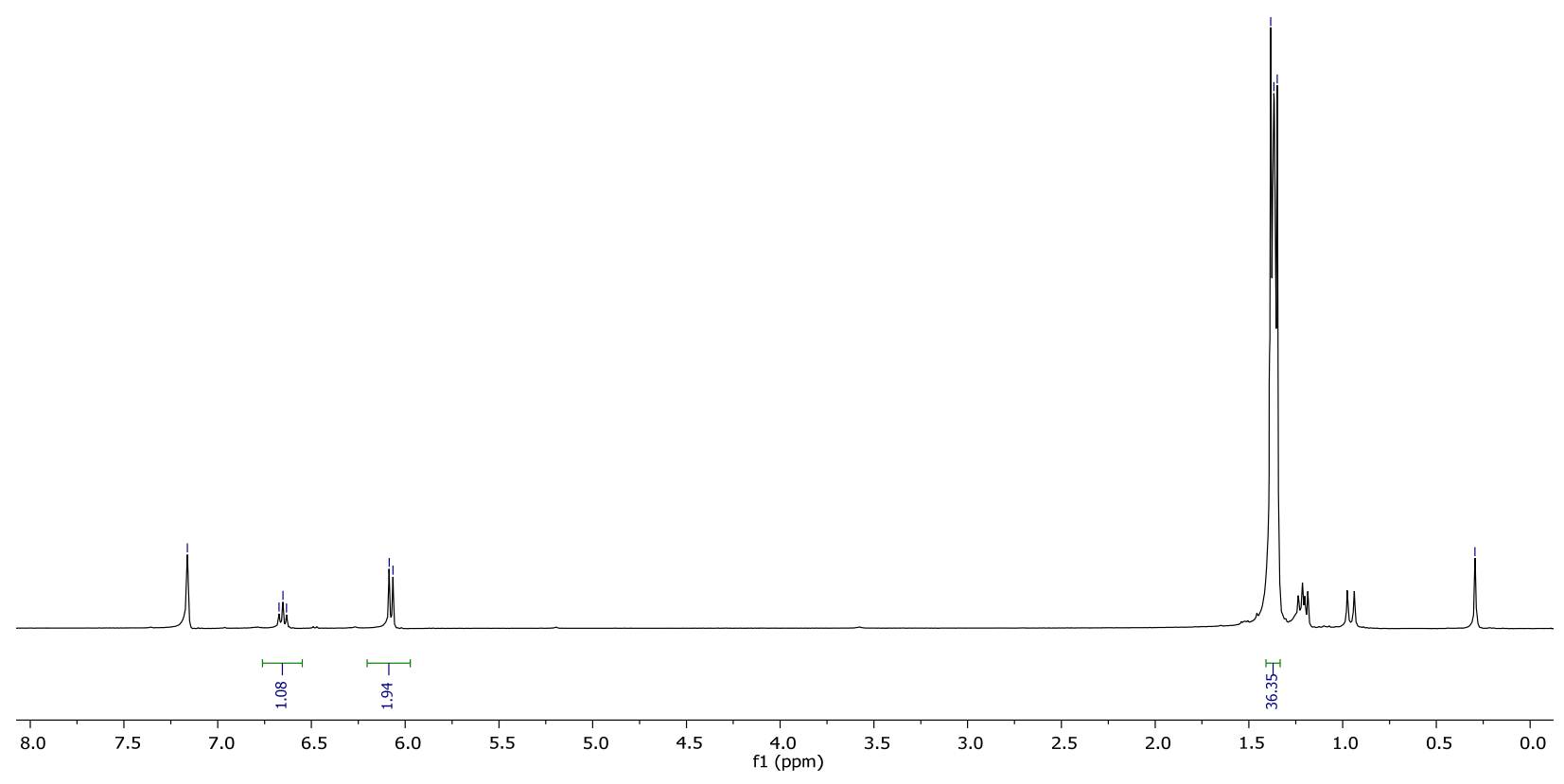

Figure $\mathbf{S} 7-{ }^{1} \mathrm{H}$ NMR of $2 \mathbf{c} \mathrm{C}_{6} \mathrm{D}_{6}$ at RT 


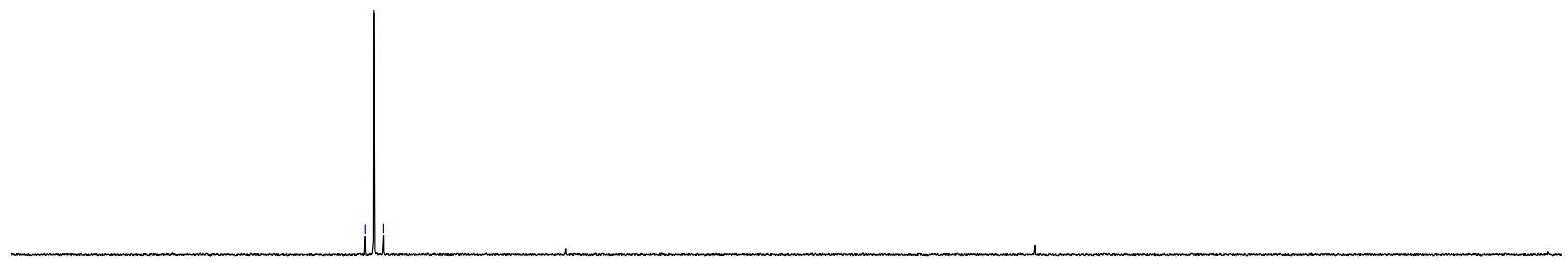

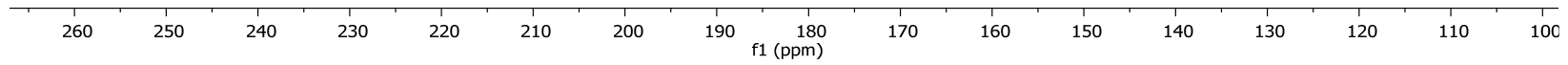

Figure S8 $-{ }^{31} \mathrm{P}\left\{{ }^{1} \mathrm{H}\right\} \mathrm{NMR} \mathrm{C}_{6} \mathrm{D}_{6}$ at $\mathrm{RT}$ for 2c 


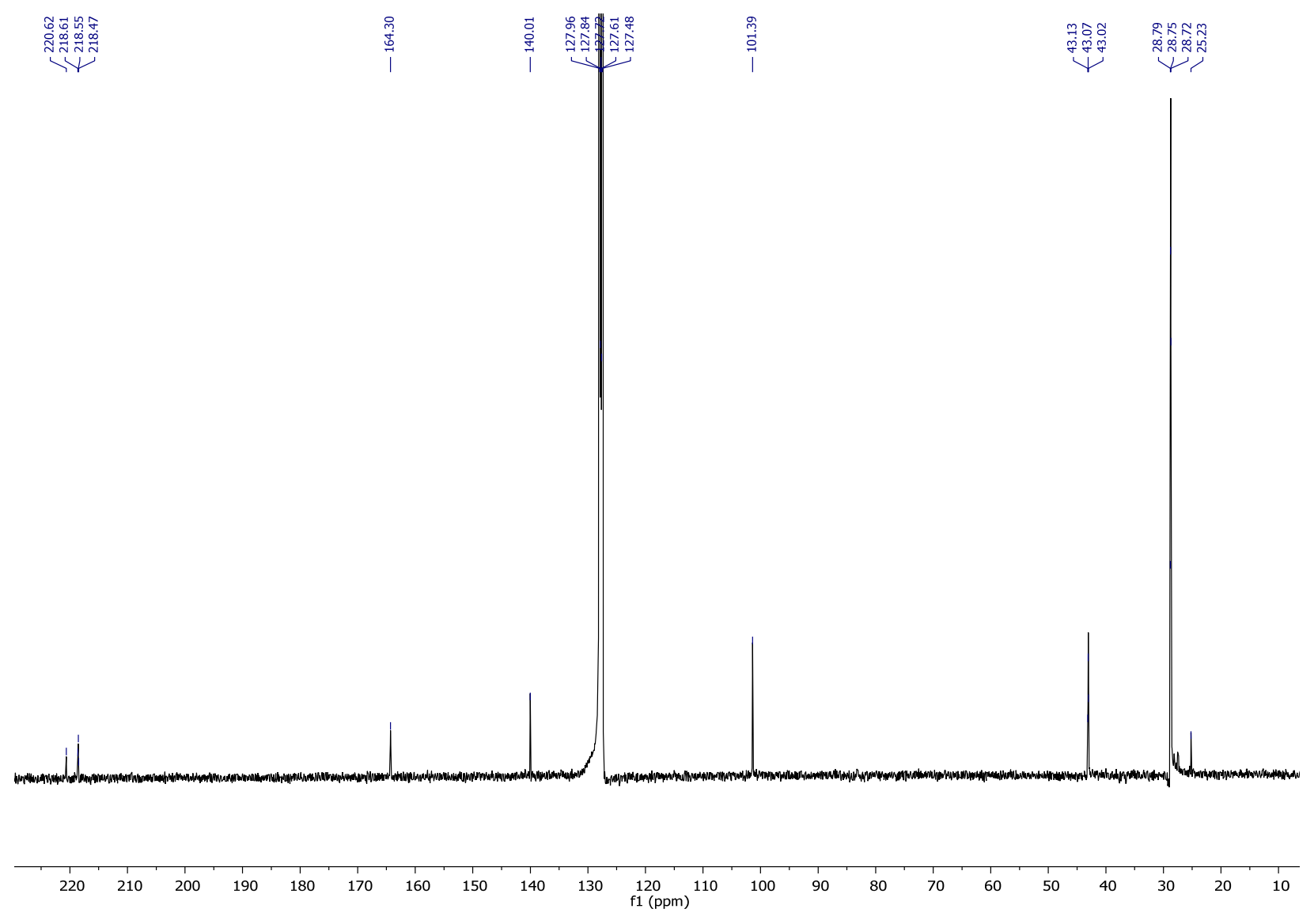

Figure S9 $-{ }^{13} \mathrm{C}\left\{{ }^{1} \mathrm{H}\right\} \quad \mathrm{NMR}$ of $2 \mathbf{c} \mathrm{C}_{6} \mathrm{D}_{6}$ at $\mathrm{RT}$ 
Characterization for Complex 2d $\left[\left({ }^{\mathrm{i}} \mathrm{PrPONOP}\right) \mathrm{W}(\mathrm{CO})_{3}\right.$

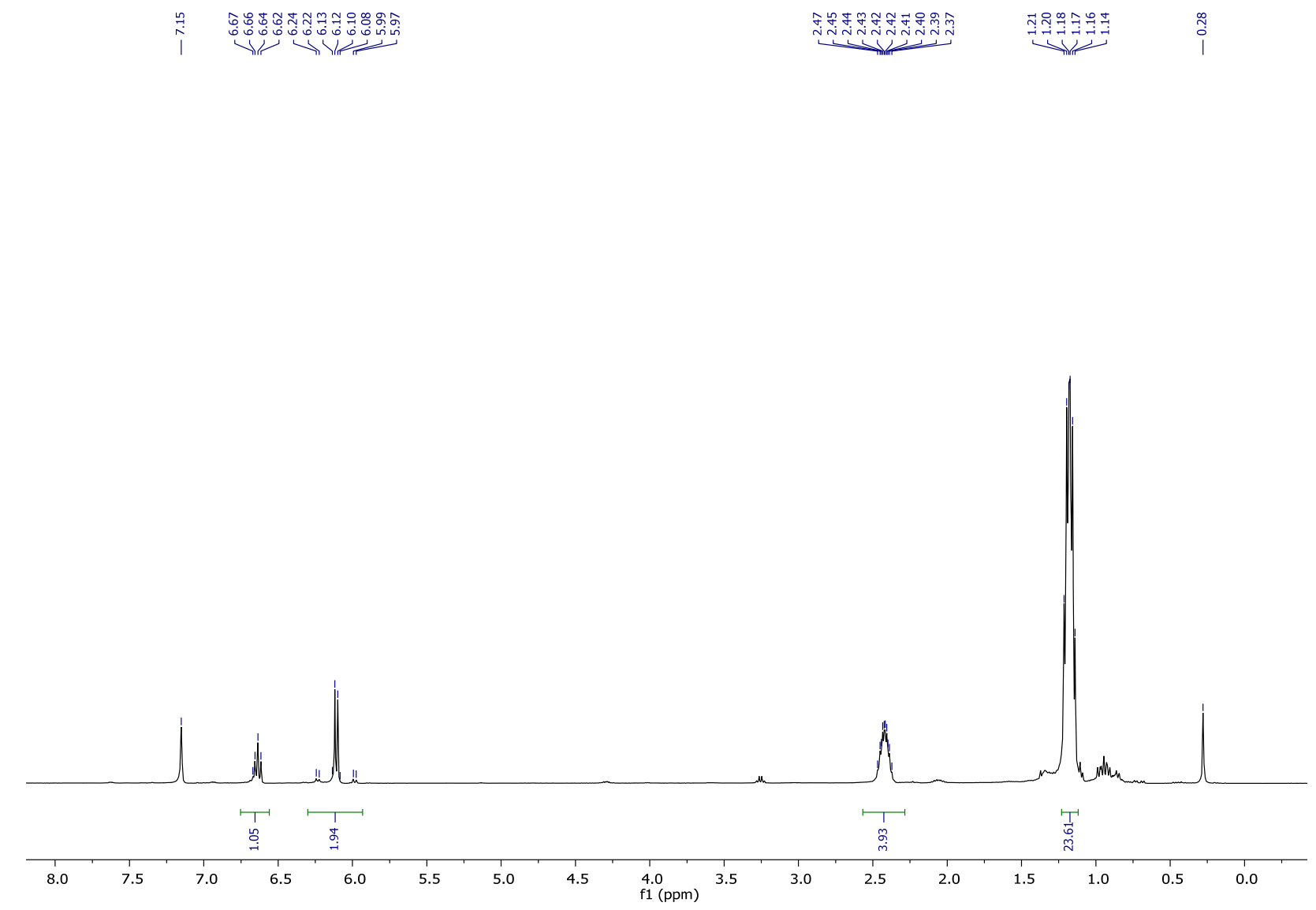

Figure S10 $-{ }^{1} \mathrm{H}$ NMR of $2 \mathrm{~d}_{6} \mathrm{D}_{6}$ at RT 


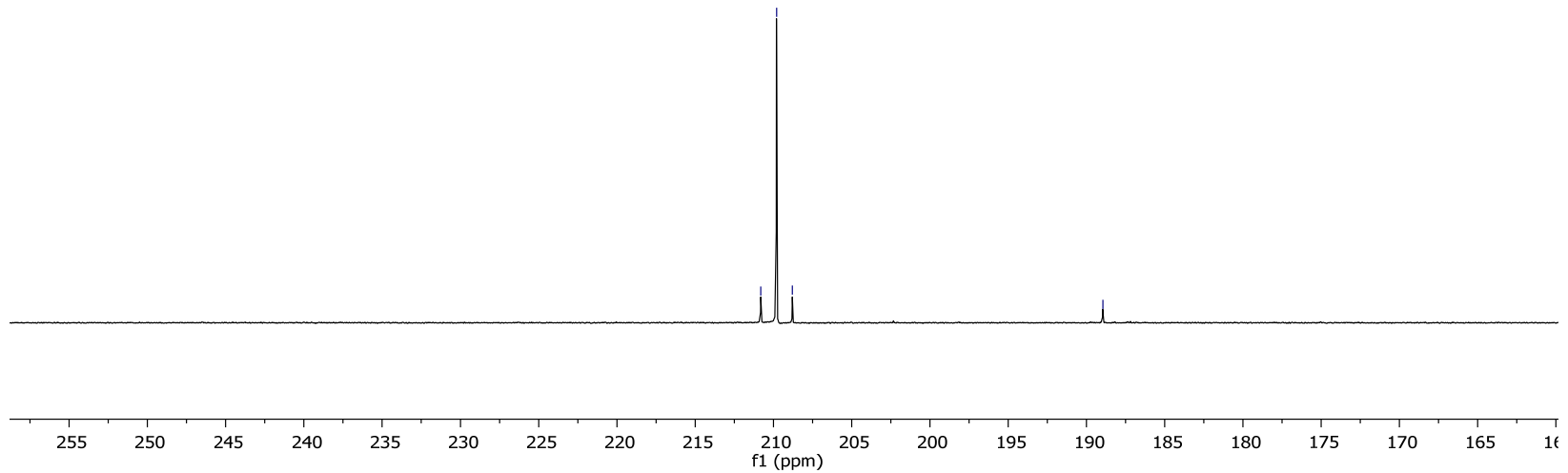

Figure S11 $-{ }^{31} \mathrm{P}\left\{{ }^{1} \mathrm{H}\right\} \mathrm{NMR} \mathrm{C}_{6} \mathrm{D}_{6}$ at $\mathrm{RT}$ for 2d 


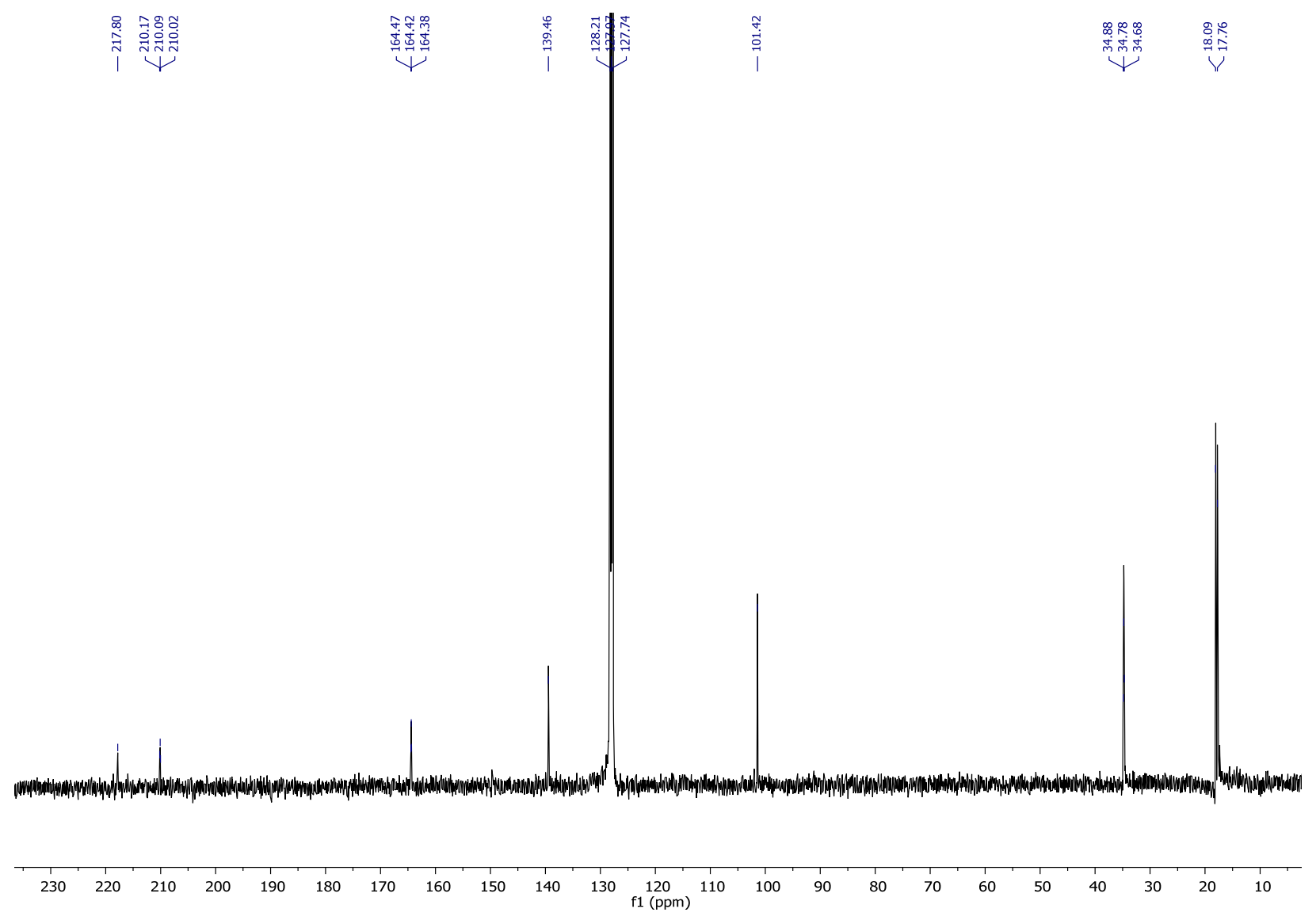

Figure S12 $-{ }^{13} \mathrm{C}\left\{{ }^{1} \mathrm{H}\right\}$ NMR of $2 \mathbf{d} \mathrm{C}_{6} \mathrm{D}_{6}$ at RT 
Characterization for Complex 3a $\left[\left({ }^{\mathrm{t}} \mathrm{BuPONOP}\right) \mathrm{Mo}(\mathrm{CO})_{3} \mathrm{H}\right] \mathrm{BF}_{4}$

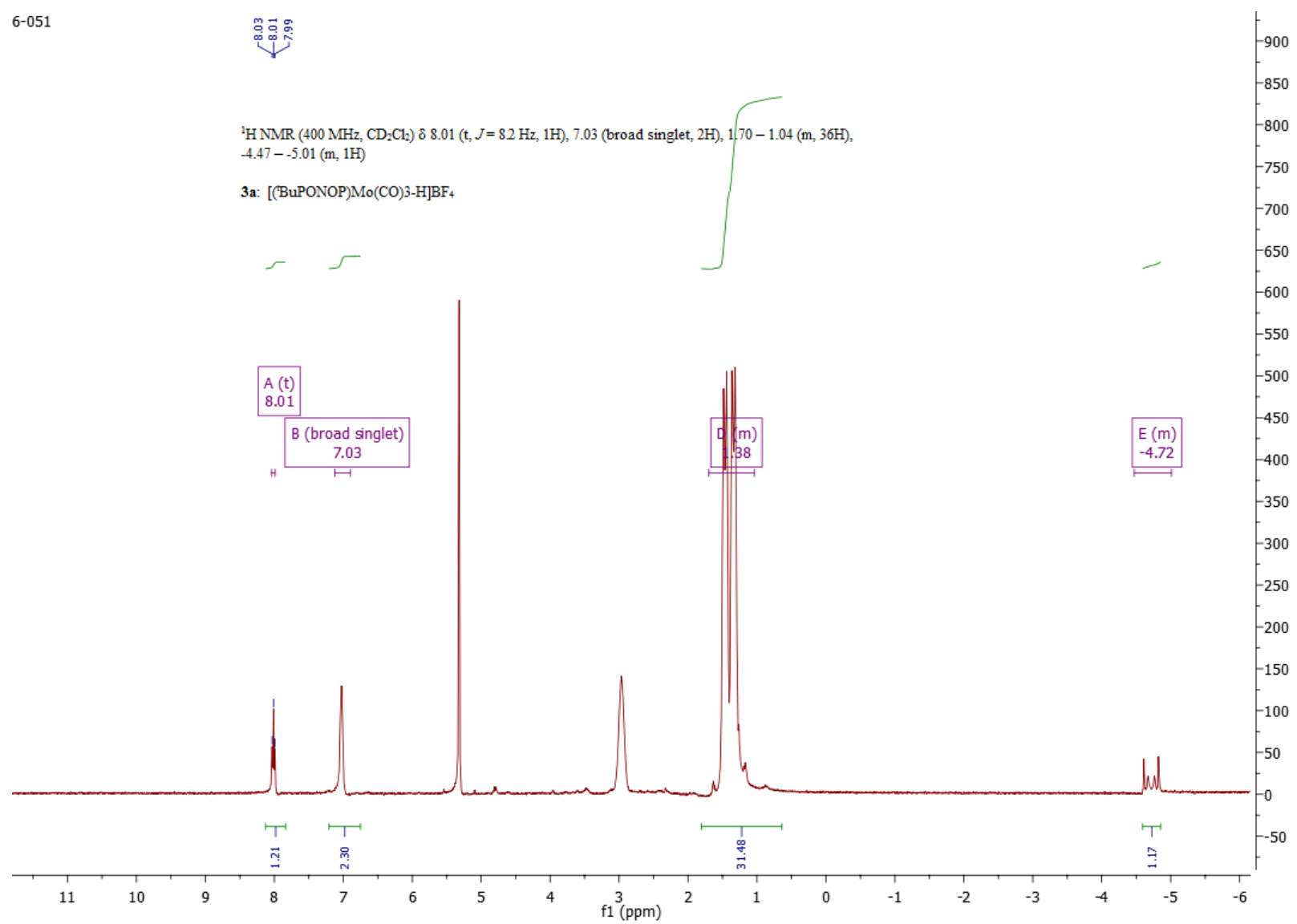

Figure S13 $-{ }^{1} \mathrm{H}$ NMR of $\mathbf{3 a}, \mathrm{CD}_{2} \mathrm{Cl}_{2}$ at $\mathrm{RT}$ 


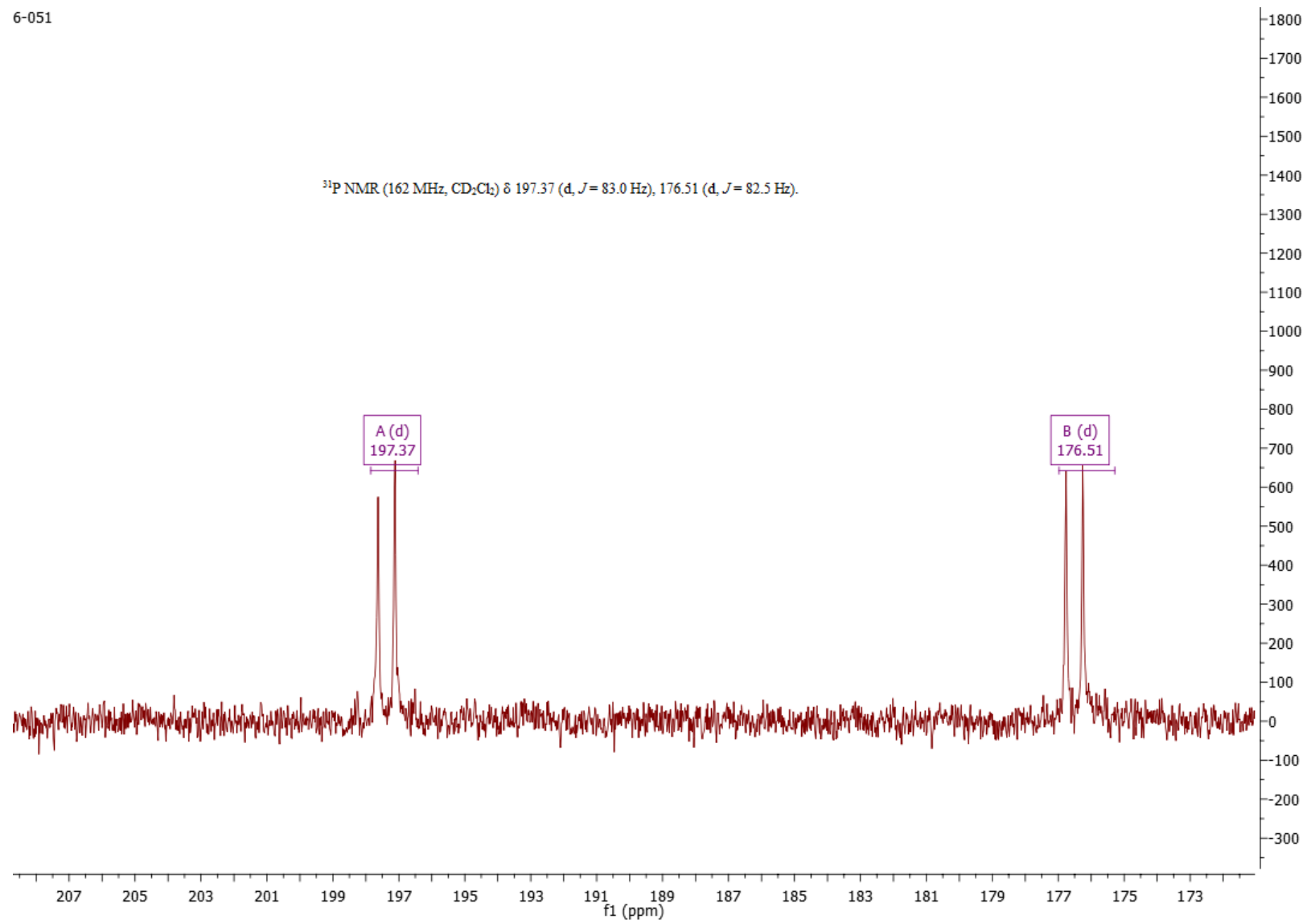

Figure $\mathbf{S 1 4}-{ }^{31} \mathrm{P}\left\{{ }^{1} \mathrm{H}\right\} \mathrm{NMR}, \mathrm{CD}_{2} \mathrm{Cl}_{2}$ at $\mathrm{RT}$ for $\mathbf{3 a}$ 


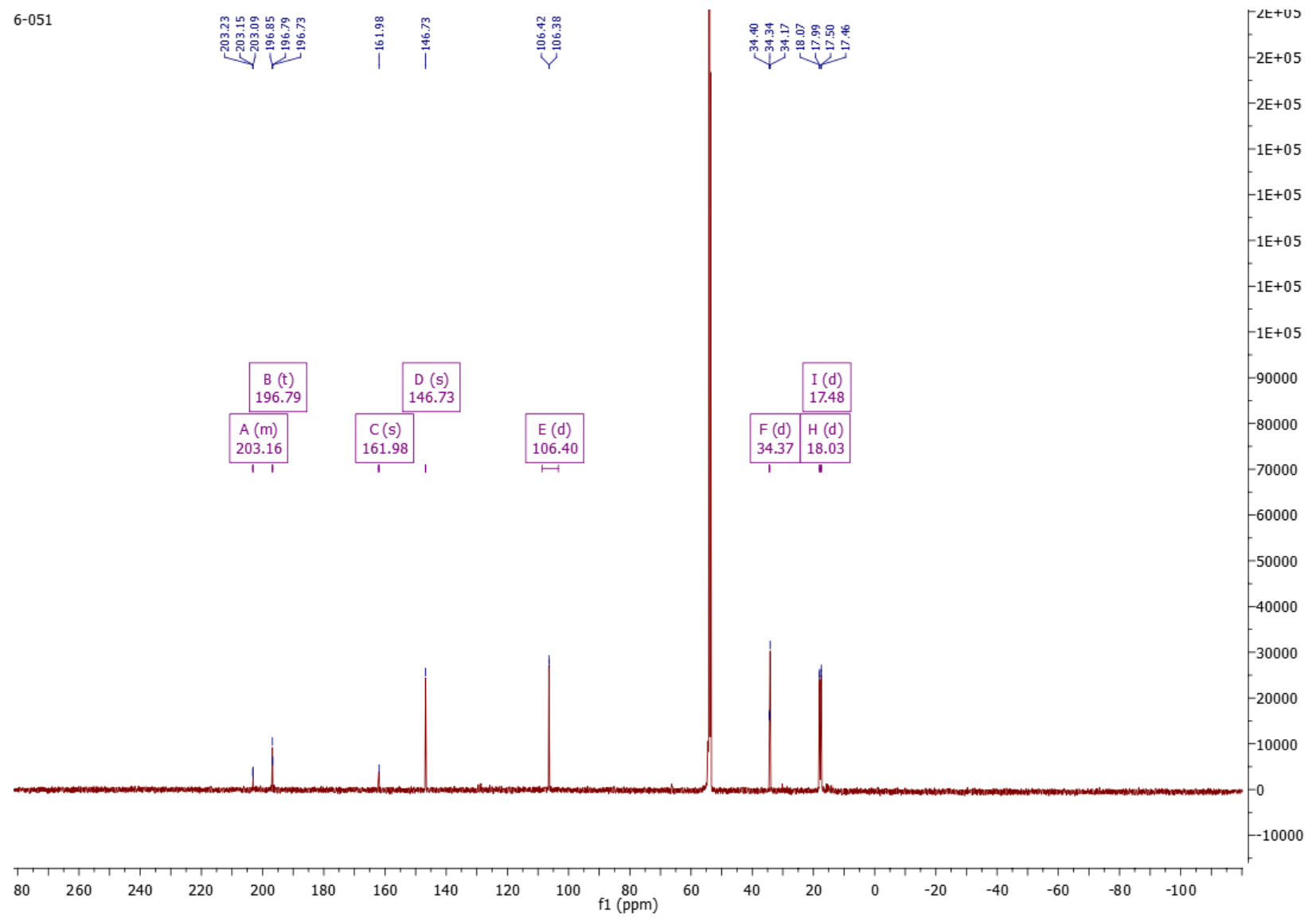

Figure $\mathbf{S 1 5}-{ }^{13} \mathrm{C}\left\{{ }^{1} \mathrm{H}\right\}$ NMR of $\mathbf{3 a}, \mathrm{CD}_{2} \mathrm{Cl}_{2}$ at RT 


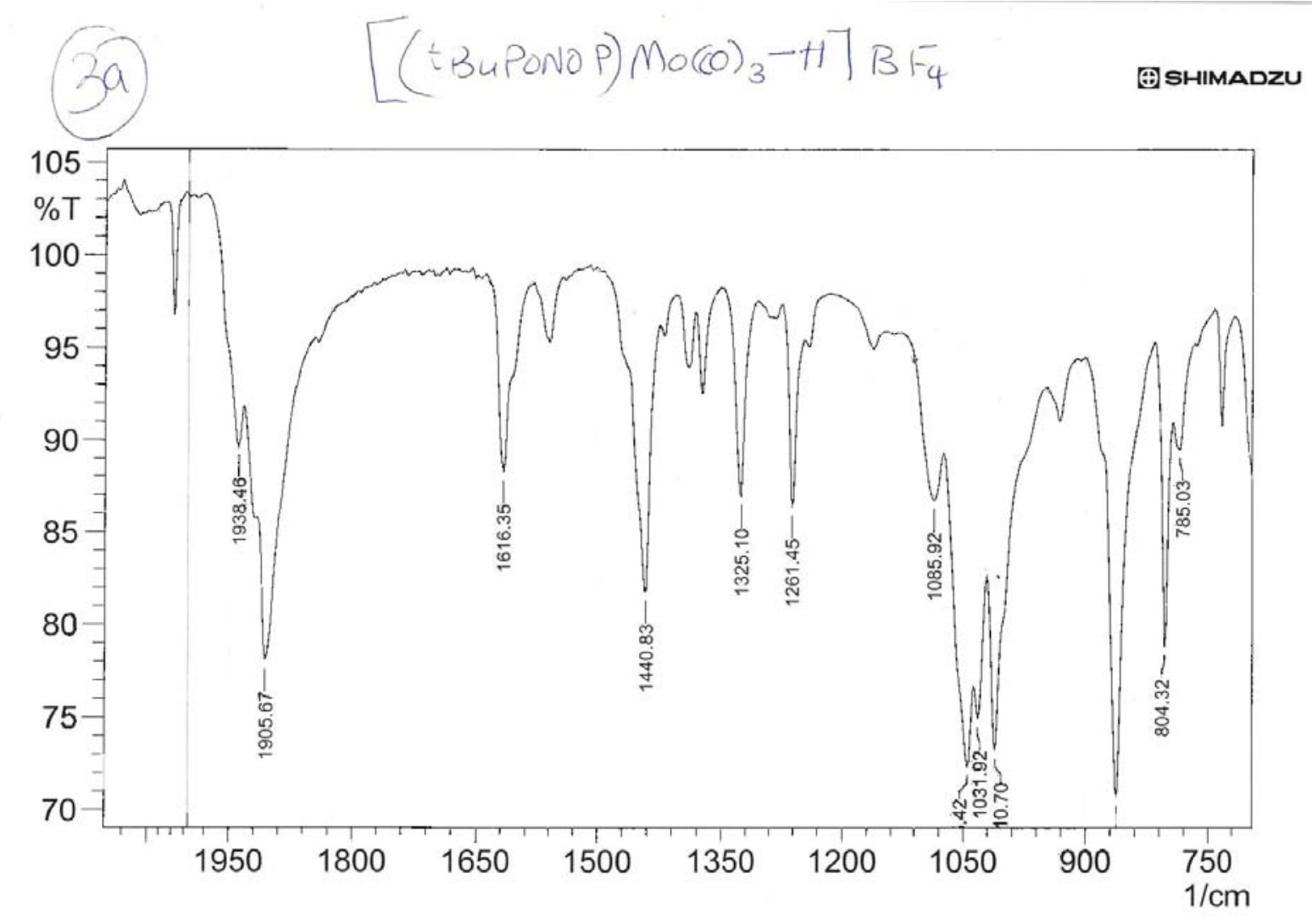

Figure S16 - Solid IR for for 3a 
Characterization for Complex $\mathbf{3 b}\left[\left({ }^{\mathrm{i}} \mathrm{PrPONOP}\right) \mathrm{Mo}(\mathrm{CO})_{3} \mathrm{H}\right] \mathrm{BF}_{4}$

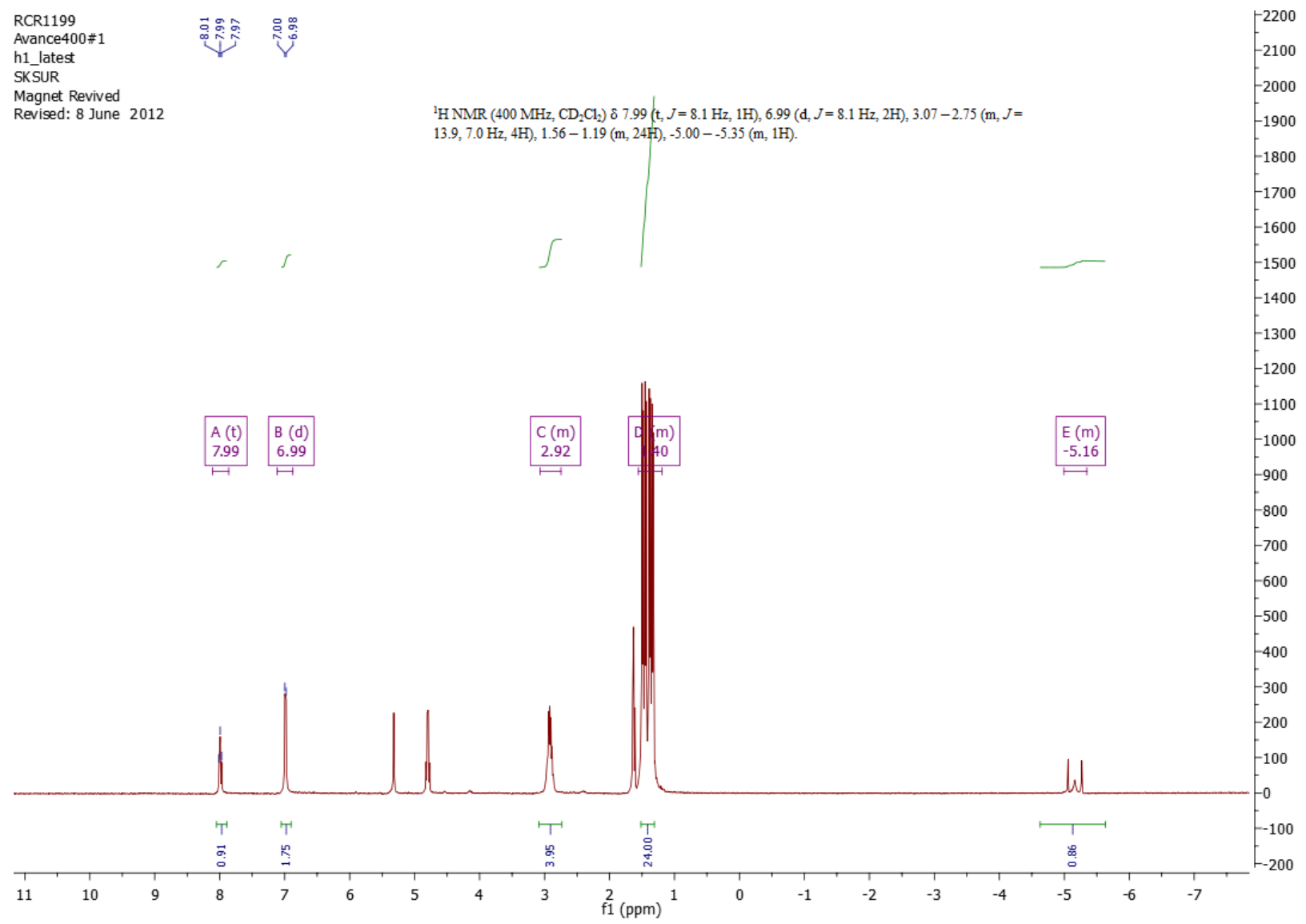

Figure $\mathbf{S 1 7}-{ }^{1} \mathrm{H}$ NMR of $\mathbf{3 b}, \mathrm{CD}_{2} \mathrm{Cl}_{2}$ at RT 


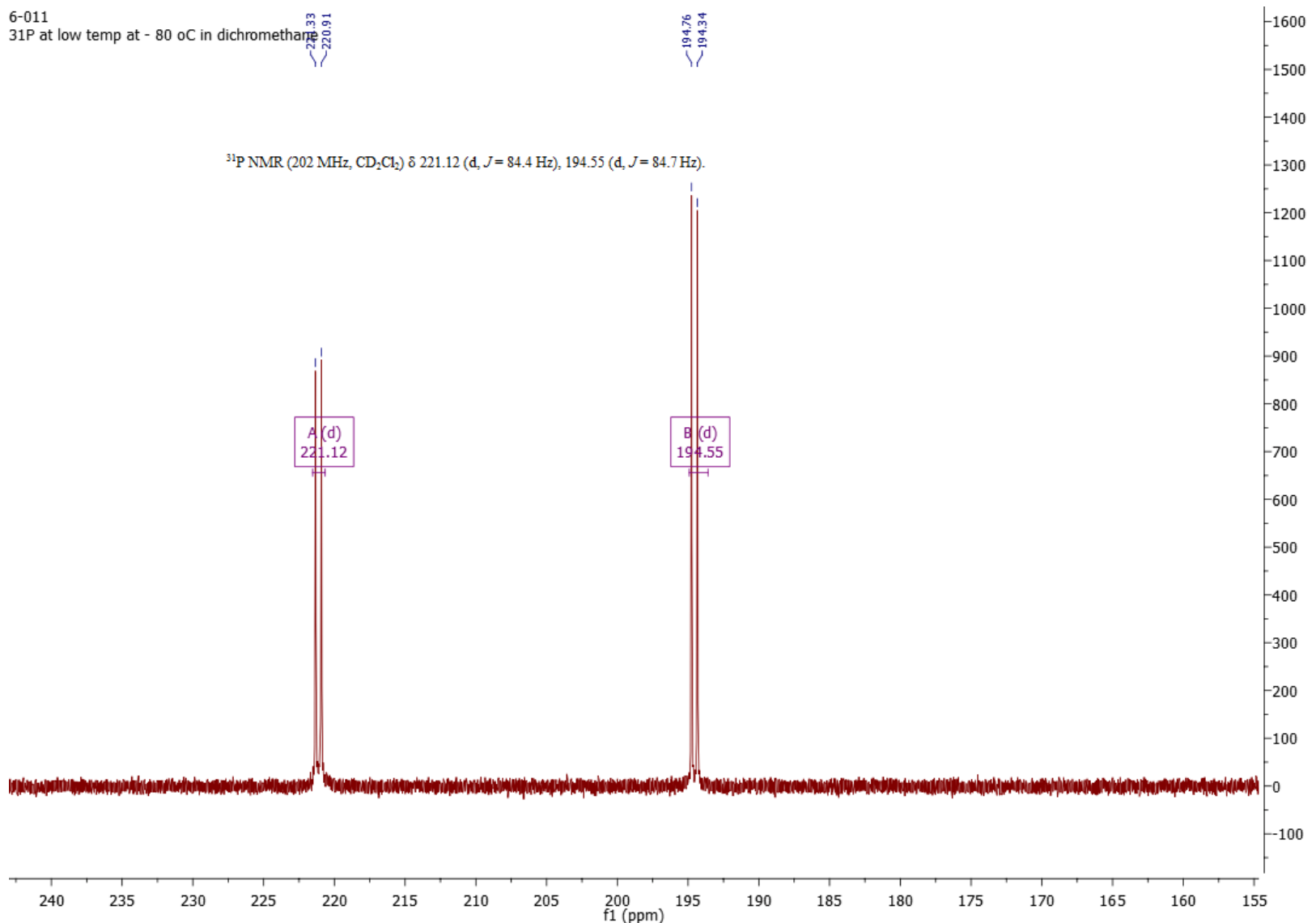

Figure $\mathbf{S 1 8}-{ }^{31} \mathrm{P}\left\{{ }^{1} \mathrm{H}\right\} \mathrm{NMR}, \mathrm{CD}_{2} \mathrm{Cl}_{2}$ at $-80{ }^{\mathbf{o}} \mathrm{C}$ for $\mathbf{3 b}$ 


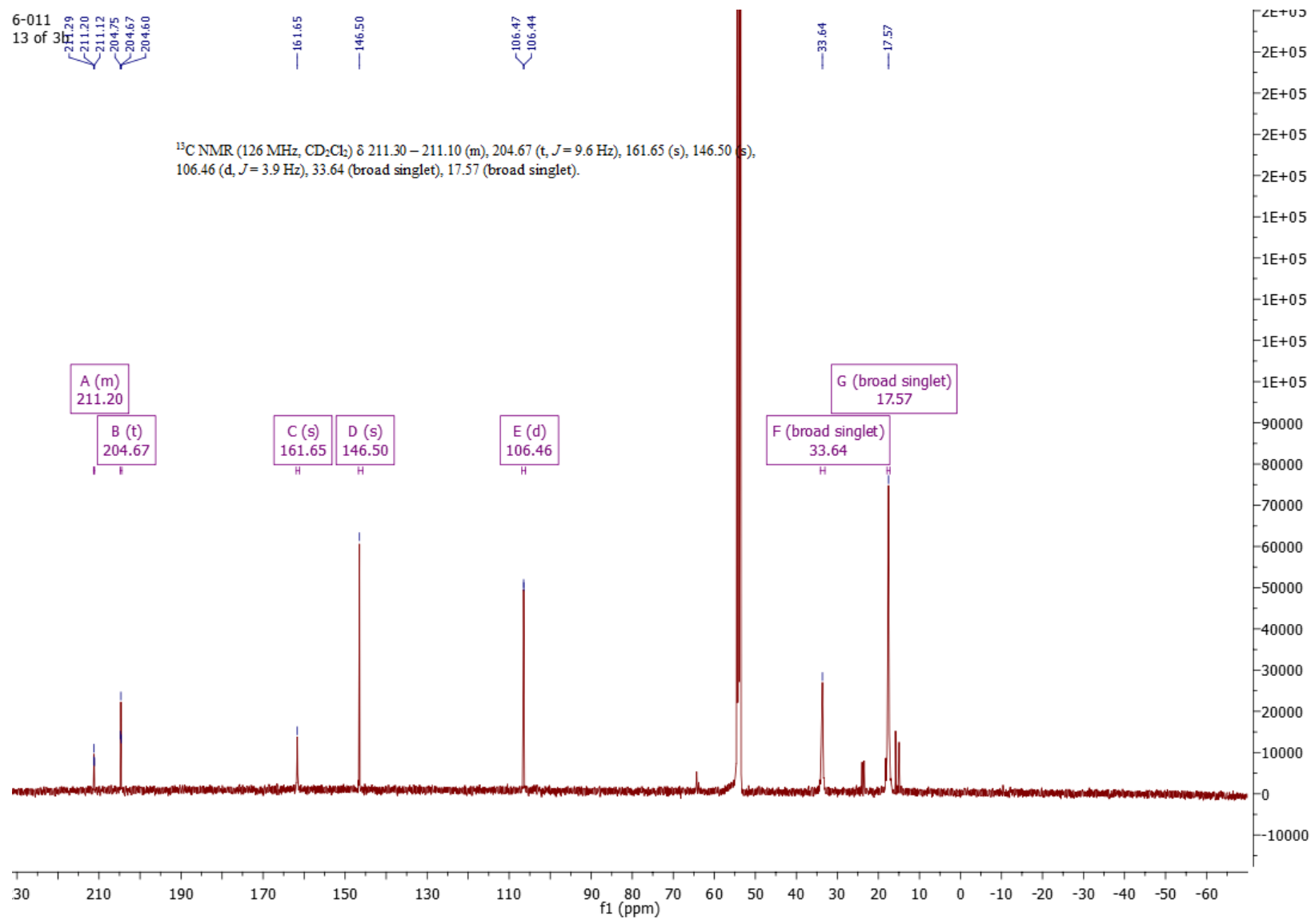

Figure $\mathbf{S 1 9}-{ }^{13} \mathrm{C}\left\{{ }^{1} \mathrm{H}\right\}$ NMR of $\mathbf{3 b}, \mathrm{CD}_{2} \mathrm{Cl}_{2}$ at RT 
ФSHIMADZU

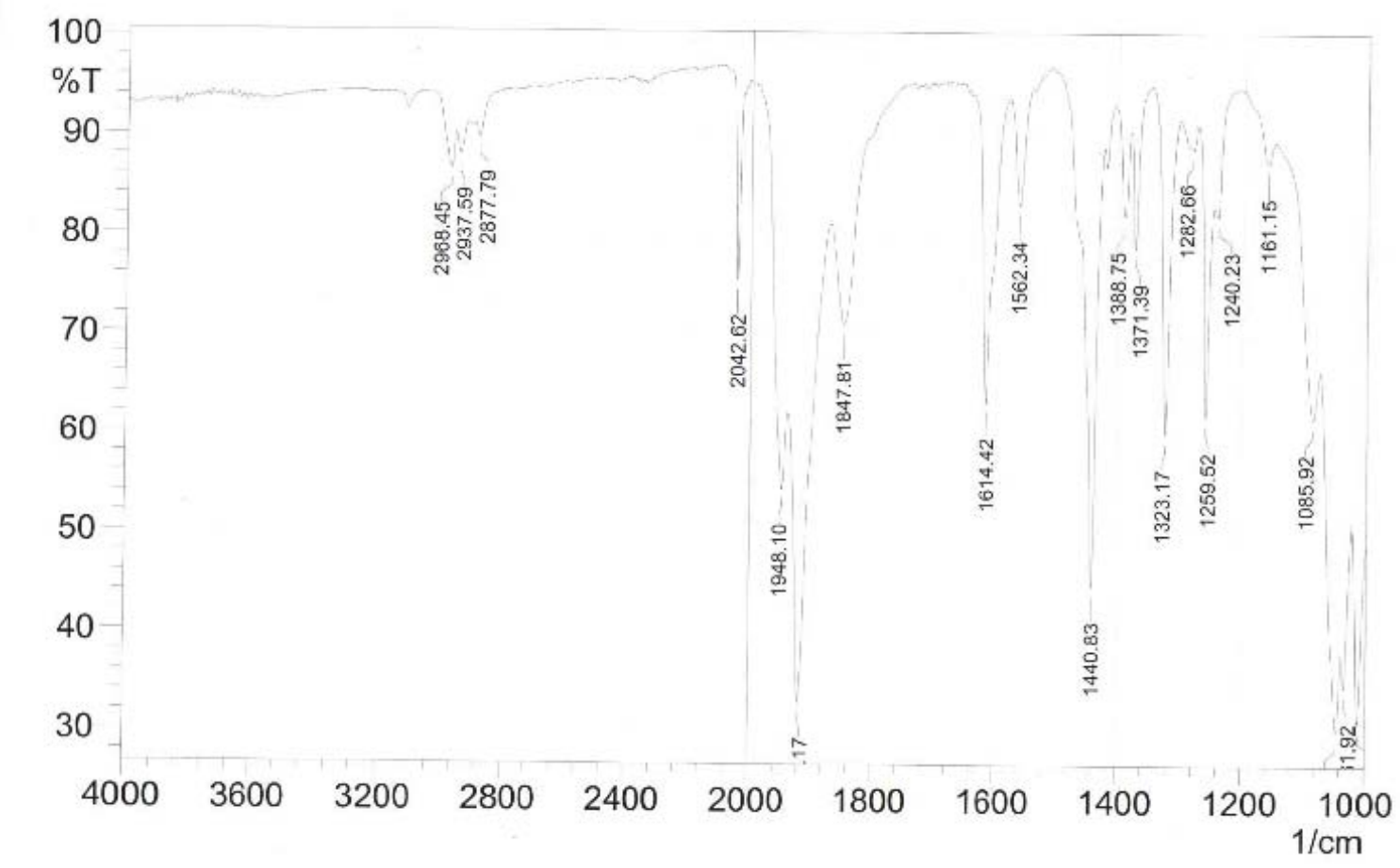

Comment;

Figure S20 - Solid IR for for $\mathbf{3 b}$ 
Characterization for Complex 3c [( $\left.\left.{ }^{\mathrm{t}} \mathrm{BuPONOP}\right) \mathrm{W}(\mathrm{CO})_{3} \mathrm{H}\right] \mathrm{BF}_{4}$

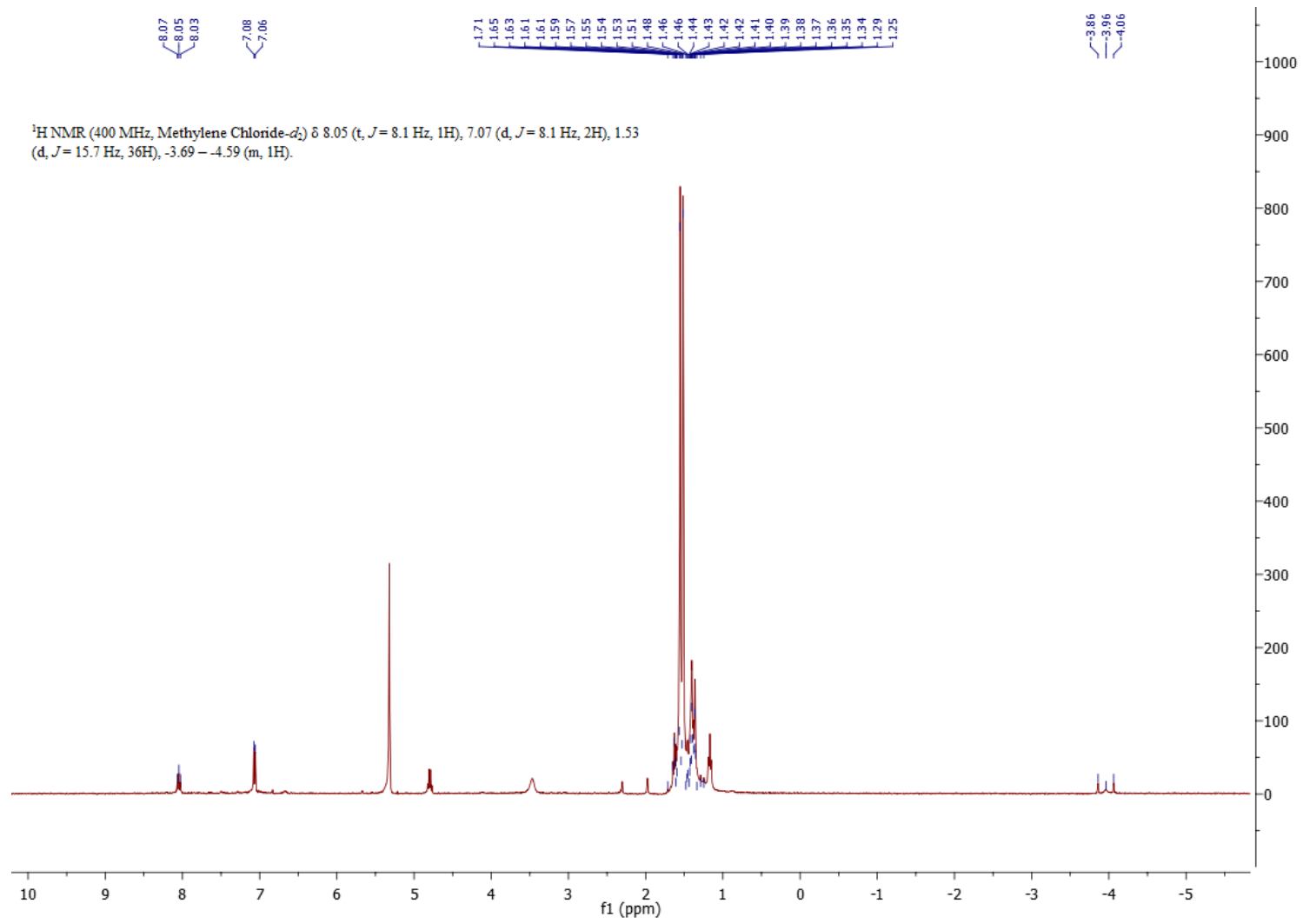

Figure S21 $-{ }^{1} \mathrm{H}$ NMR of $\mathbf{3 c}, \mathrm{CD}_{2} \mathrm{Cl}_{2}$ at $\mathrm{RT}$ 


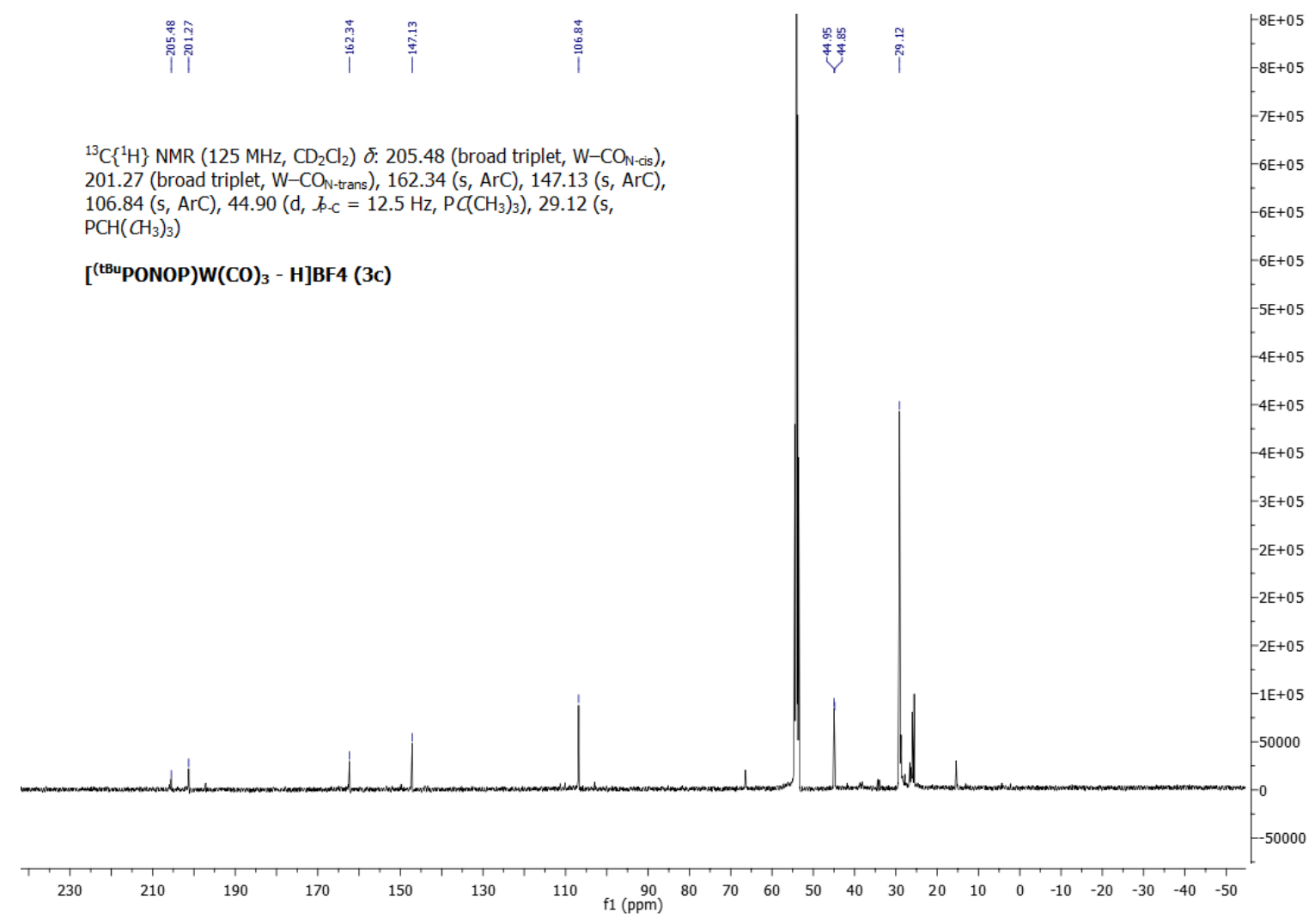

Figure $\mathbf{S 2 2}-{ }^{13} \mathrm{C}\left\{{ }^{1} \mathrm{H}\right\}$ NMR of $\mathbf{3 c}, \mathrm{CD}_{2} \mathrm{Cl}_{2}$ at $\mathrm{RT}$ 


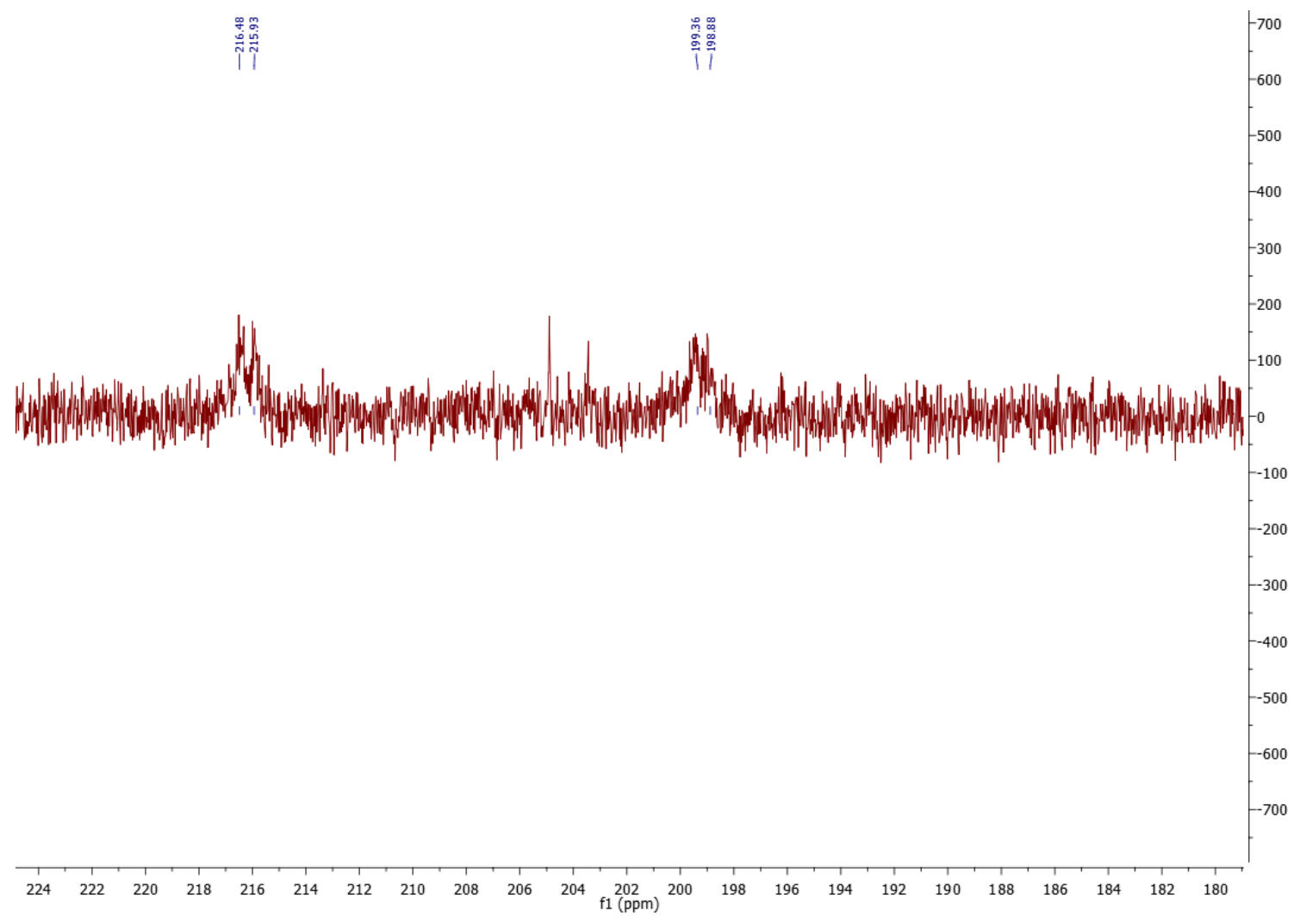

Figure $\mathbf{S 2 3}-{ }^{31} \mathrm{P}\left\{{ }^{1} \mathrm{H}\right\} \mathrm{NMR}, \mathrm{CD}_{2} \mathrm{Cl}_{2}$ at $\mathrm{RT}$ for $\mathbf{3 c}$ 


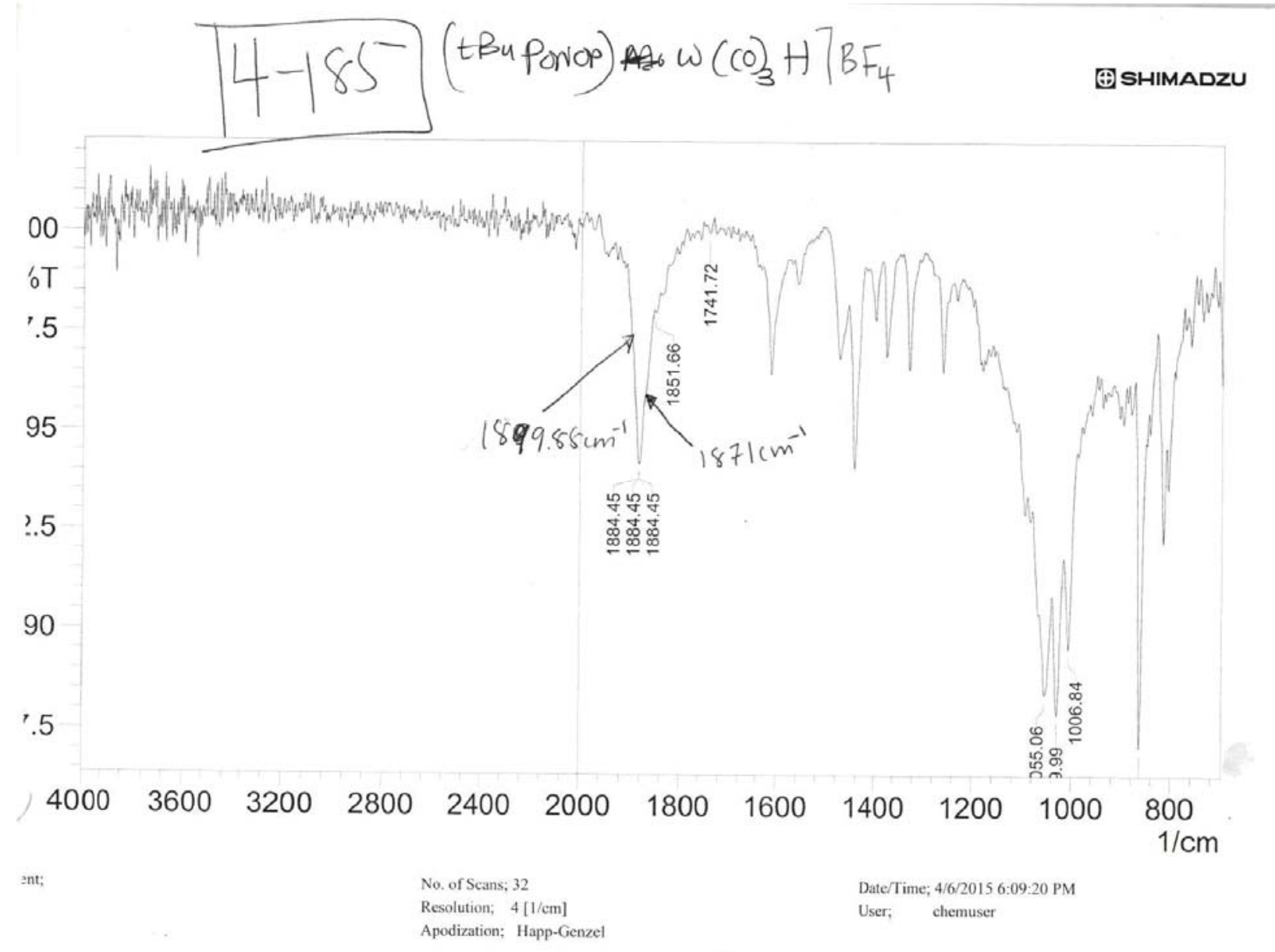

Figure S24 - Solid IR for for 3c 
Characterization for Complex 3d [( $\left.\left.{ }^{\mathrm{i}} \mathrm{PrPONOP}\right) \mathrm{W}(\mathrm{CO})_{3} \mathrm{H}\right] \mathrm{BF}_{4}$

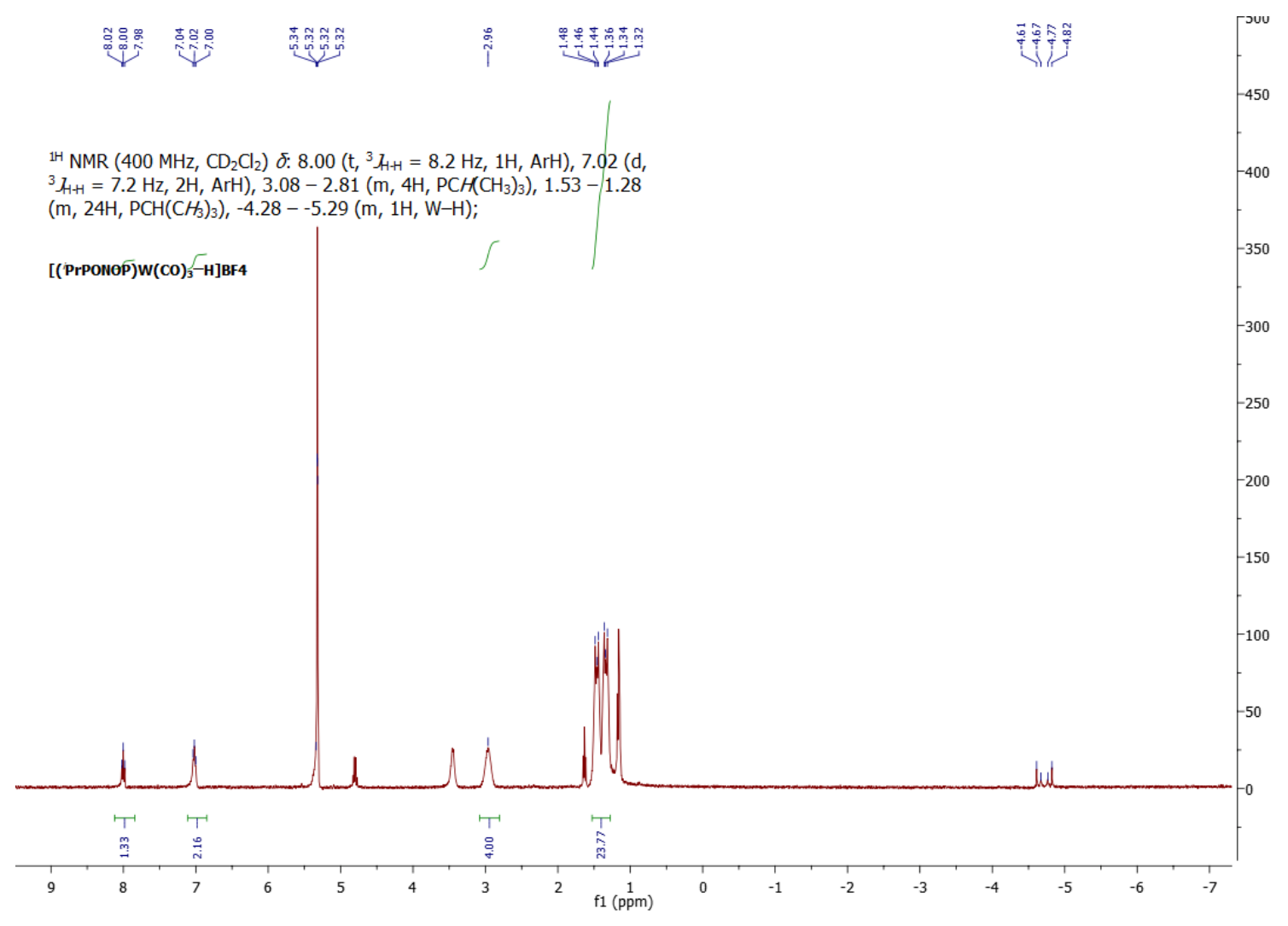

Figure $\mathbf{S 2 5}-{ }^{1} \mathrm{H}$ NMR of $\mathbf{3 d}, \mathrm{CD}_{2} \mathrm{Cl}_{2}$ at RT 


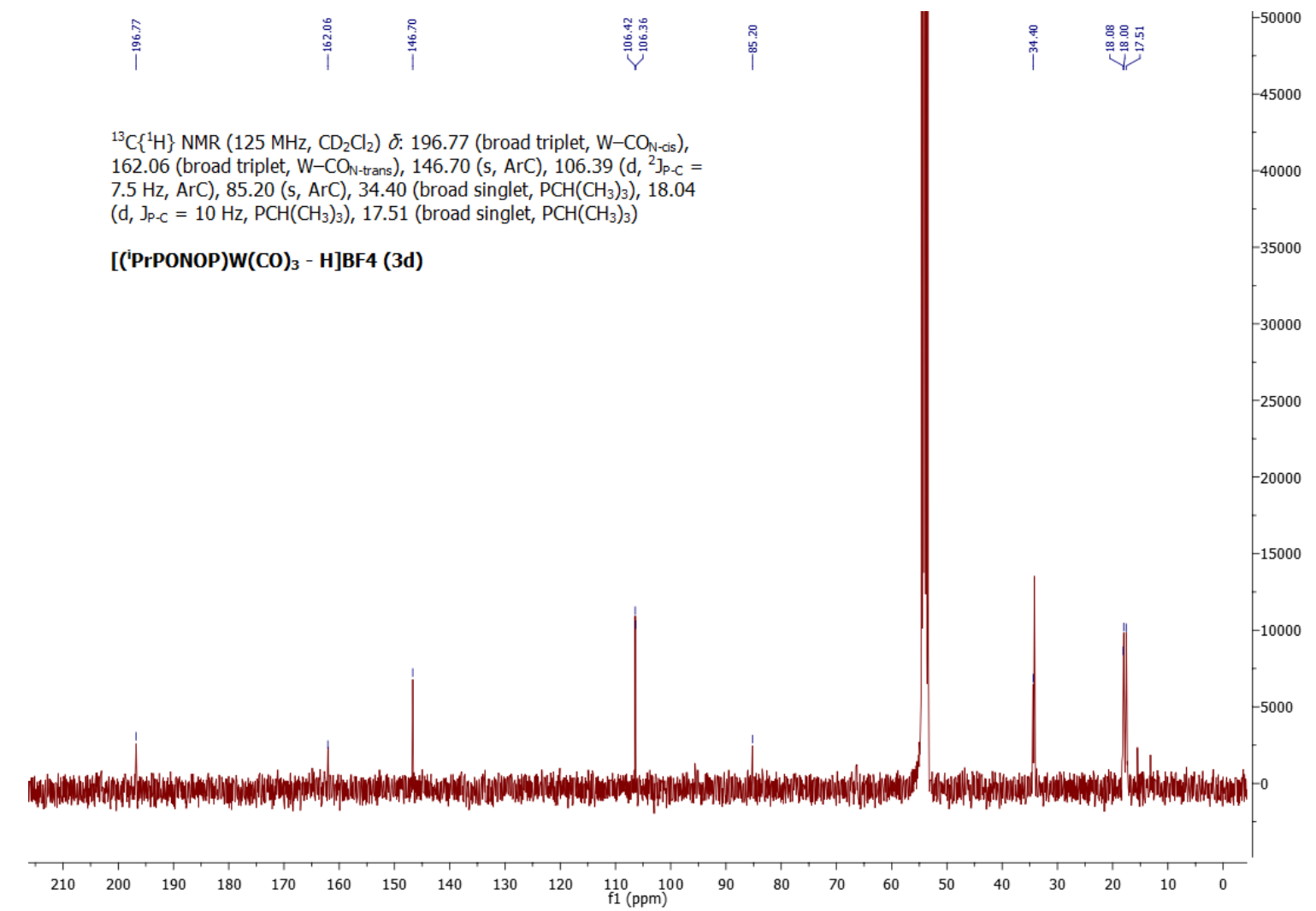

Figure S26 $-{ }^{13} \mathrm{C}\left\{{ }^{1} \mathrm{H}\right\}$ NMR of $\mathbf{3 d}, \mathrm{CD}_{2} \mathrm{Cl}_{2}$ at RT 


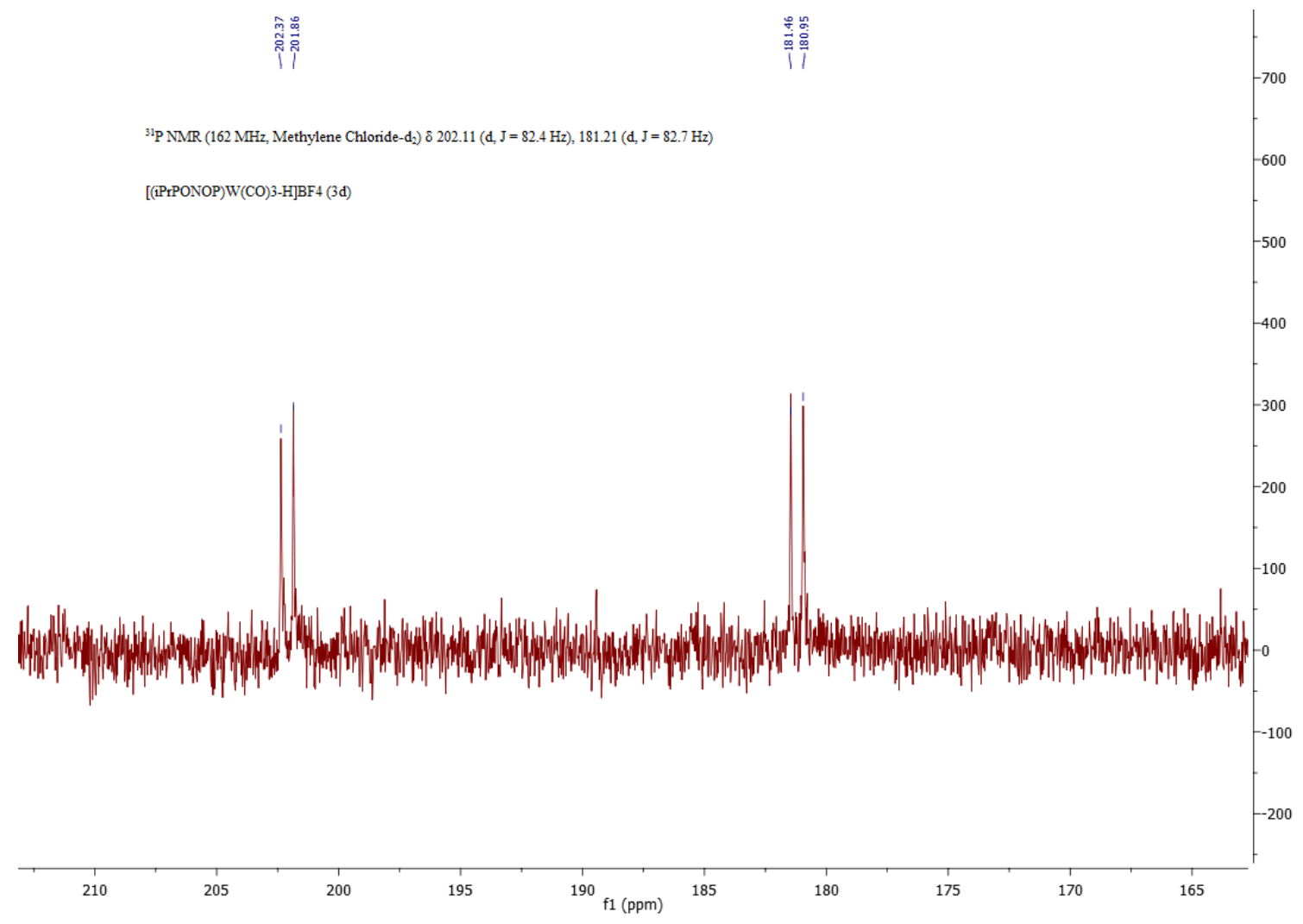

Figure $\mathbf{S 2 7}-{ }^{31} \mathrm{P}\left\{{ }^{1} \mathrm{H}\right\} \mathrm{NMR}, \mathrm{CD}_{2} \mathrm{Cl}_{2}$ at $\mathrm{RT}$ for $\mathbf{3 d}$ 


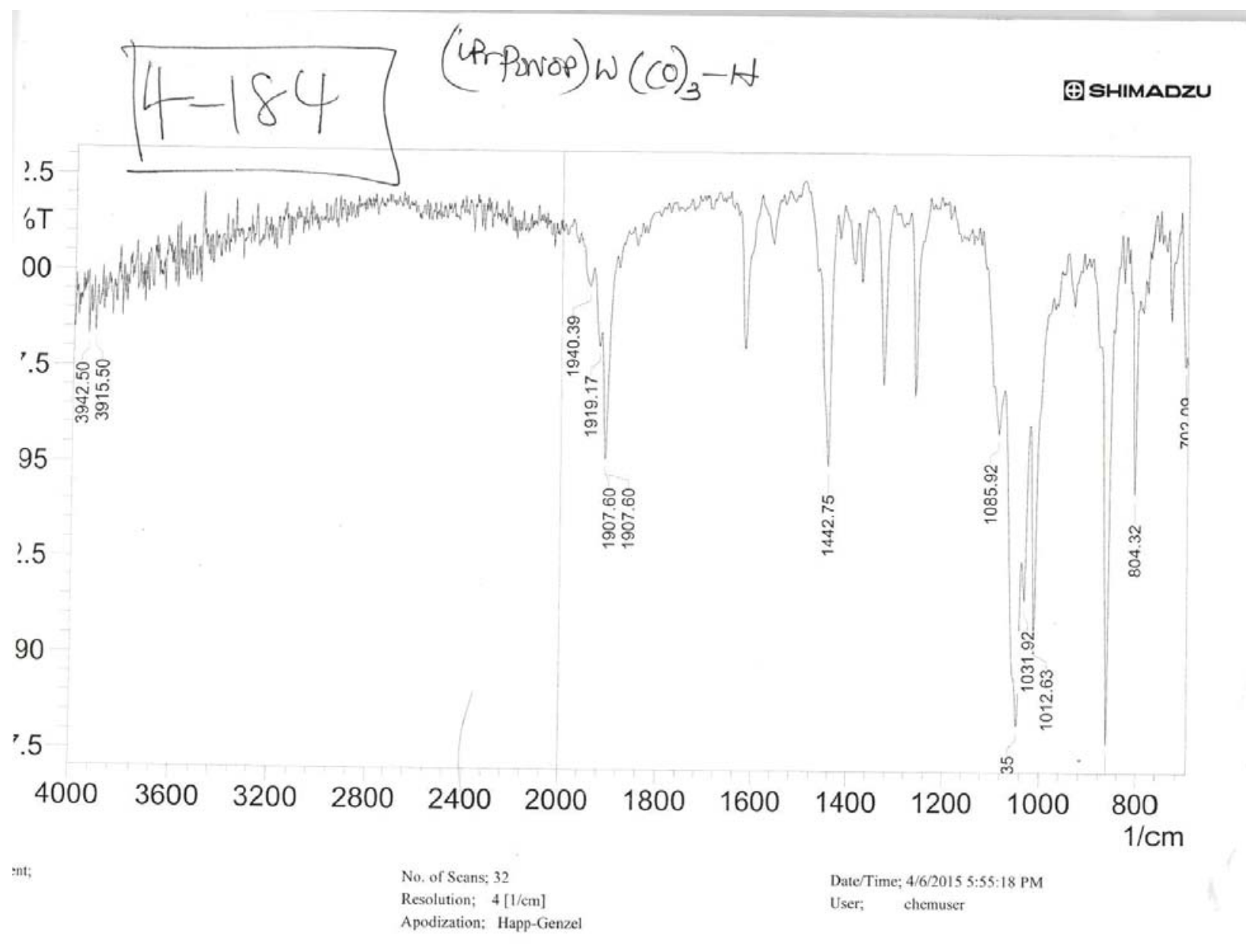

Figure S28 - Solid IR for for 3d 
$\underbrace{\mathrm{CO}}_{\mathrm{O}-\mathrm{P}}$ $\left(\mathrm{Pr}_{2}\right)$

3b

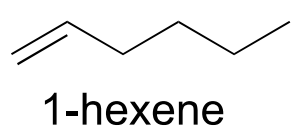
(1 equiv)

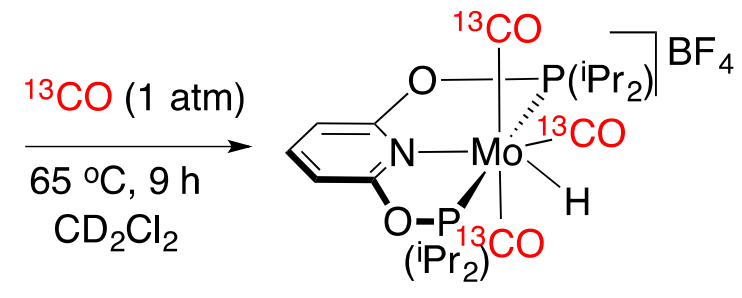
observed

+1 -hexene isomers
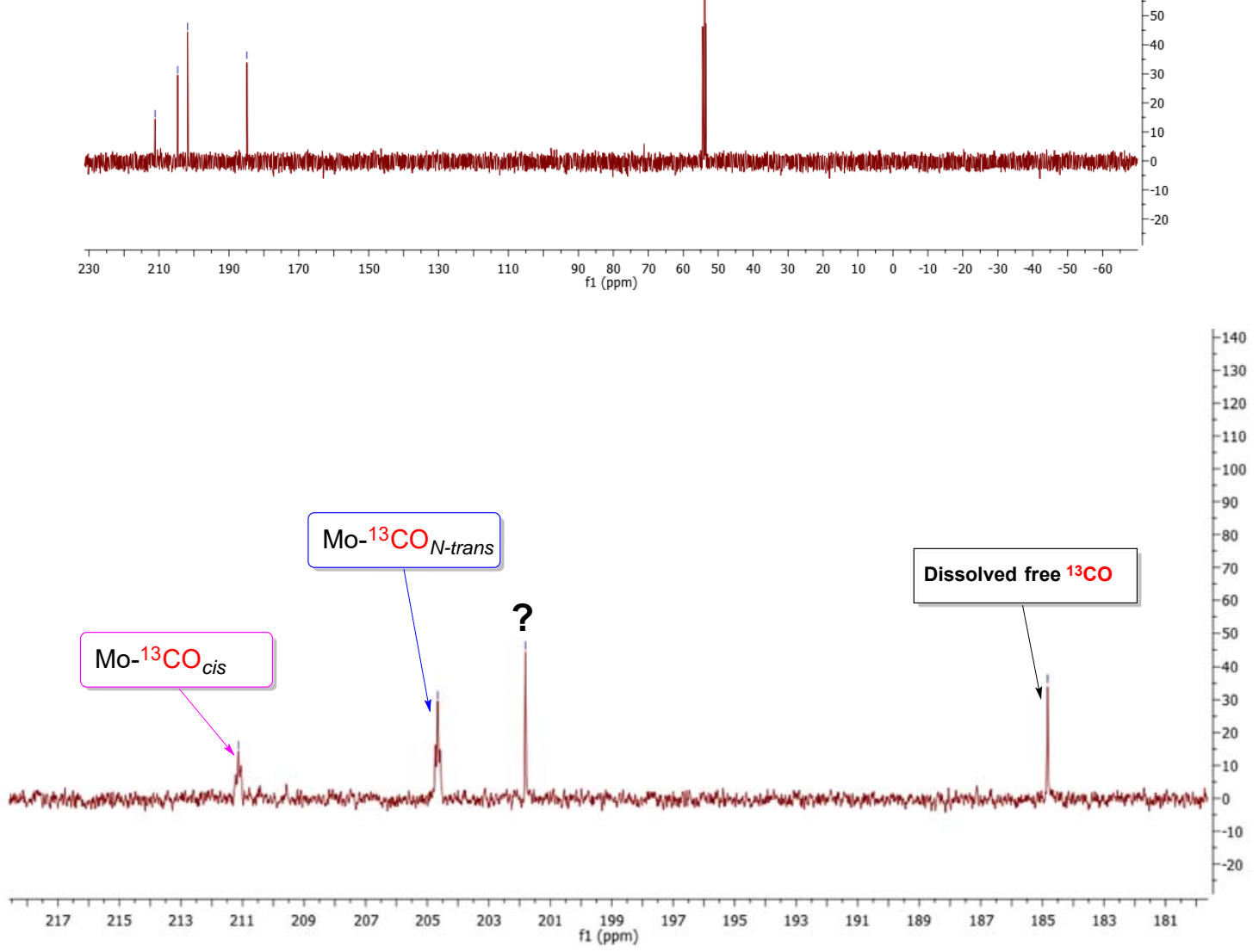

Figure S29: ${ }^{13} \mathrm{CO}$ incorporation in the $\mathbf{3 b}$ complex during reaction 
Isomerization of 1-hexene with $10 \mathrm{~mol} \%$ of $\mathbf{3 b}$.
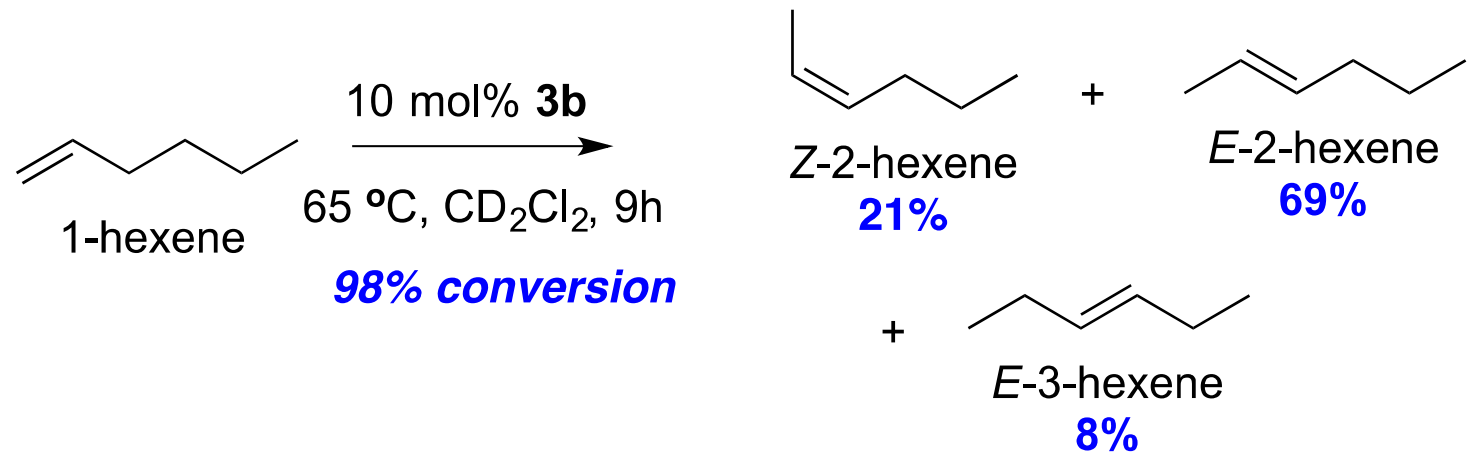

Conditions: $[\mathbf{3 b}]=32 \mathrm{mM}$, [1-hexene $]=328 \mathrm{mM}$, [internal standard- 1,4 dibromobenzene $]=$ $328 \mathrm{mM}, 0.5 \mathrm{~mL}$ of $\mathrm{CD}_{2} \mathrm{Cl}_{2}, 9 \mathrm{~h}$ at $65^{\circ} \mathrm{C}$ 

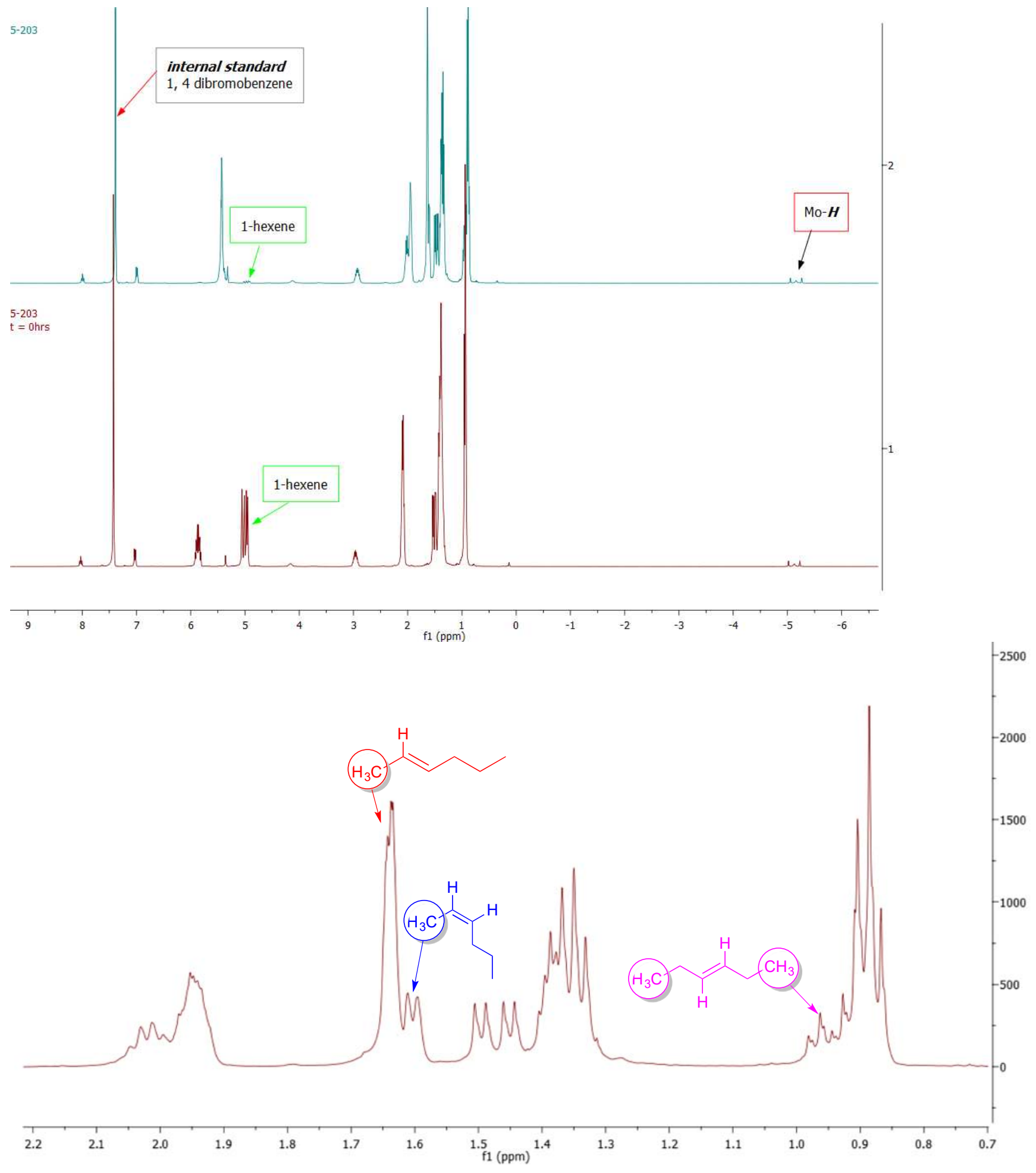

Figure S30 - (top) ${ }^{1} \mathrm{H}$ NMR in $\mathrm{CD}_{2} \mathrm{Cl}_{2}$ showing reaction mixture at $\mathrm{t}=0 \mathrm{hr}$ and $\mathrm{t}=9 \mathrm{hr}$ (b) ${ }^{1} \mathrm{H}$ NMR spectra showing major isomer products after $9 \mathrm{~h}$. 


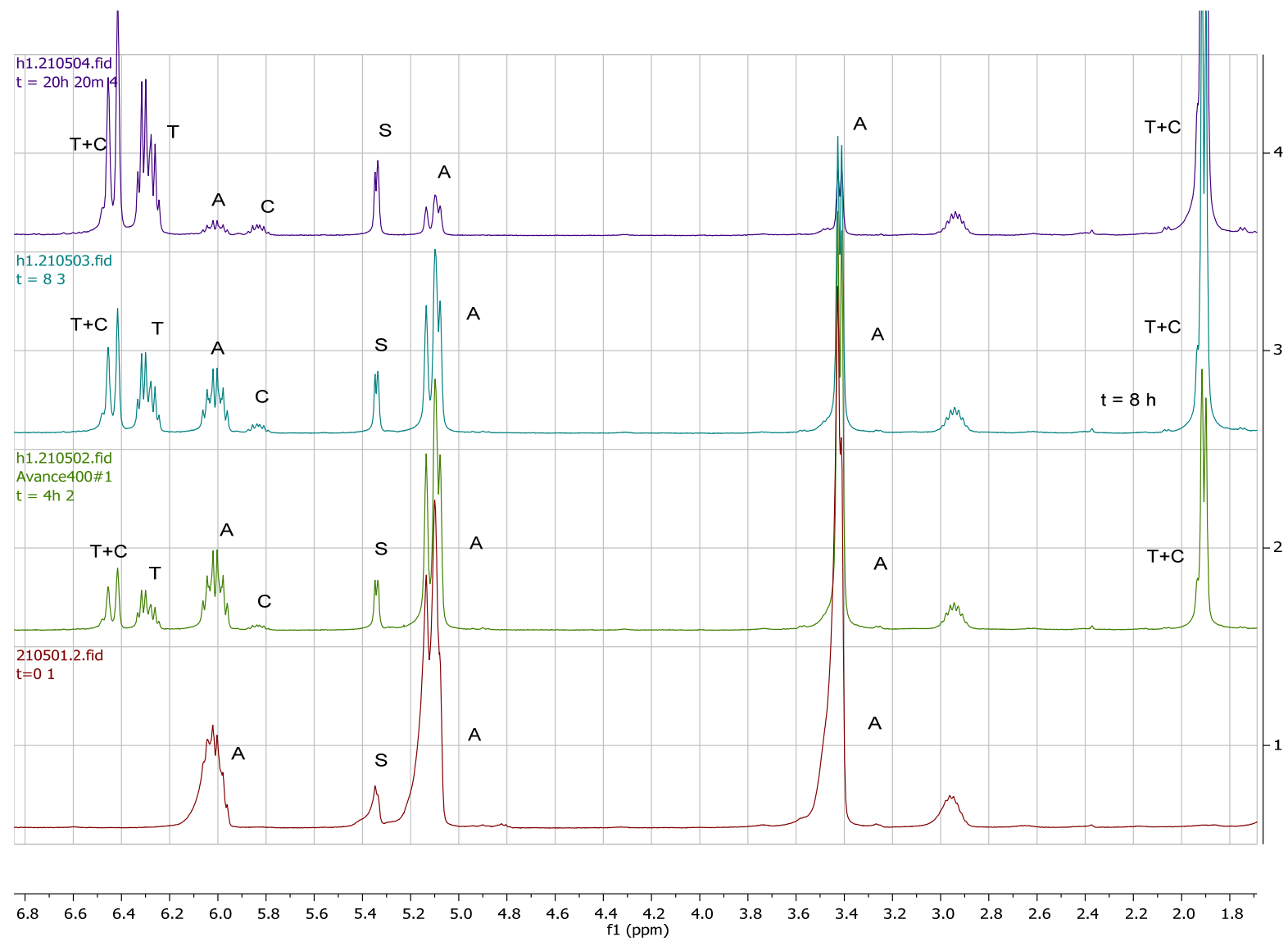

Figure S-31. ${ }^{1} \mathrm{H}$ NMR spectra of the catalytic isomerization of allylbenzene (A) to trans- $\beta$ methylstyrene (B) and cis-B-methylstyrene (C) by $\left[\left({ }^{\mathrm{iPr}} \mathrm{PONOP}\right) \mathrm{Mo}(\mathrm{CO})_{3} \mathrm{H}\right]^{+} \mathrm{BF}_{4}^{-}, \mathbf{3 b}$, in $\mathrm{CD}_{2} \mathrm{Cl}_{2}$ at $65^{\circ} \mathrm{C}$. $\mathrm{S}=$ solvent residual protons. [allylbenzene] $=0.29 \mathrm{M}$. 7\% catalyst loading.

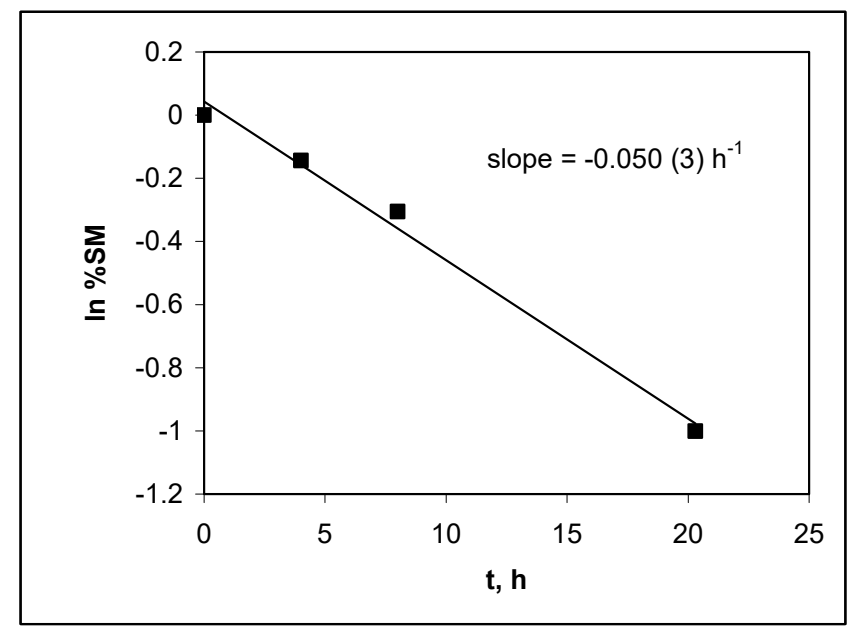

Figure S-32. First order plot for the isomerization of allylbenzene to $\beta$-methylstyrene using ${ }^{1} \mathrm{H}$ NMR data for Me resonances. 
(a)
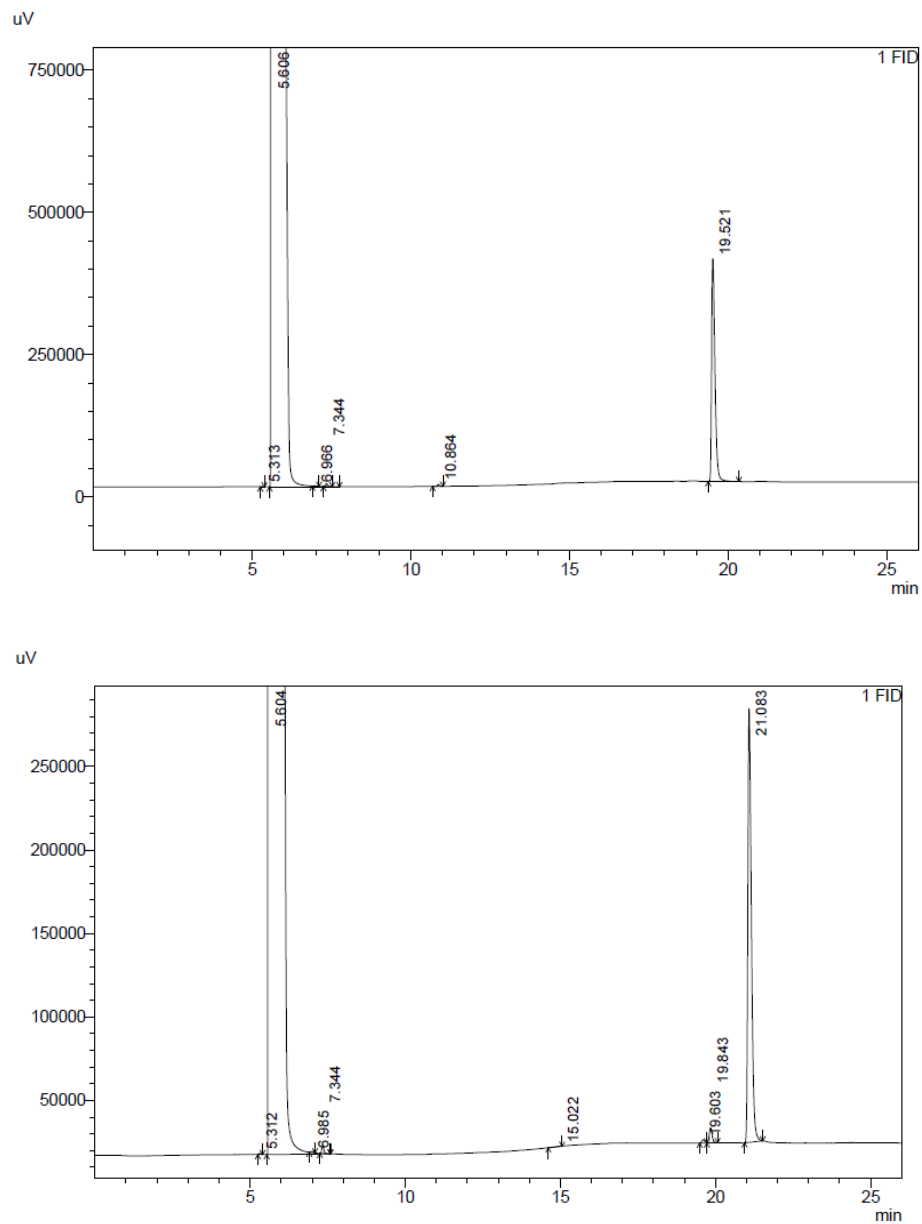

(b)

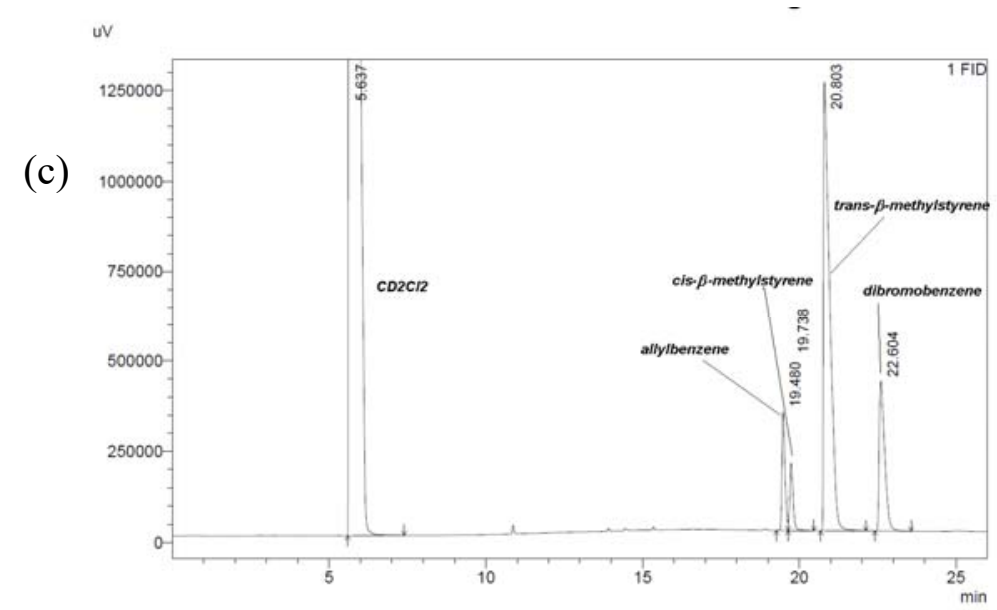

Figure S-33. GC traces of the catalytic isomerization of allylbenzene (A) to trans- $\beta$ methylstyrene (B) and cis-B-methylstyrene by $\left[\left({ }^{\mathrm{iPr}} \mathrm{PONOP}\right) \mathrm{Mo}(\mathrm{CO})_{3} \mathrm{H}\right]^{+} \mathrm{BF}_{4}^{-}, \mathbf{3 b}$, in $\mathrm{CD}_{2} \mathrm{Cl}_{2}$ at $65{ }^{\circ} \mathrm{C}$. [allylbenzene] $=0.29 \mathrm{M}$. $7 \%$ catalyst loading. (a) allylbenzene in $\mathrm{CH}_{2} \mathrm{Cl}_{2}$, (b) trans- $\beta$ allylbenzene (Aldrich) in $\mathrm{CH}_{2} \mathrm{Cl}_{2}$, (c) reaction mixture in $\mathrm{CD}_{2} \mathrm{Cl}_{2}$. Shimadzu GC-2010 with Agilent GS-GASPRO PLOT column (30m x $0.32 \mathrm{~mm}$ ID), 100-250 ${ }^{\circ} \mathrm{C} @ 10 \% \mathrm{~min}$, column flow: $2 \mathrm{~mL} / \mathrm{min}, 25: 1$ split. 


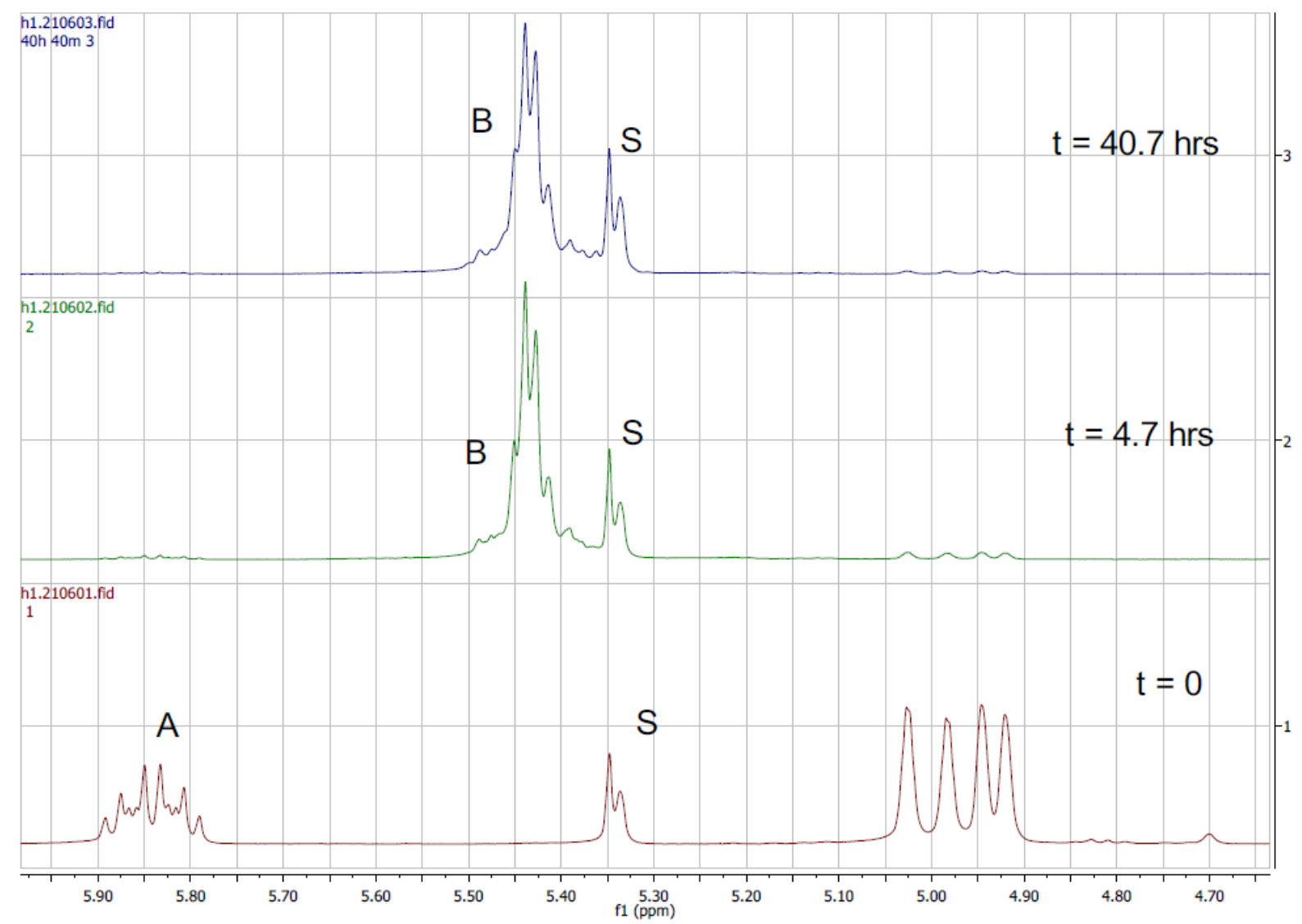

Figure S-34. ${ }^{1} \mathrm{H}$ NMR spectra of the catalytic isomerization of 1-octene to internal octenes by $\left.\left[{ }^{\mathrm{iPr}} \mathrm{PONOP}\right) \mathrm{Mo}(\mathrm{CO})_{3} \mathrm{H}\right]^{+} \mathrm{BF}_{4}^{-}, 3 \mathbf{b}$, in $\mathrm{CD}_{2} \mathrm{Cl}_{2}$ at $65^{\circ} \mathrm{C}$. [1-octene $=0.22 \mathrm{M}$. $7 \%$ catalyst loading. $\mathrm{A}=1$-octene vinyl resonances; $\mathrm{B}=$ internal octane vinyl resonances; $\mathrm{S}=$ residual solvent resonances. 


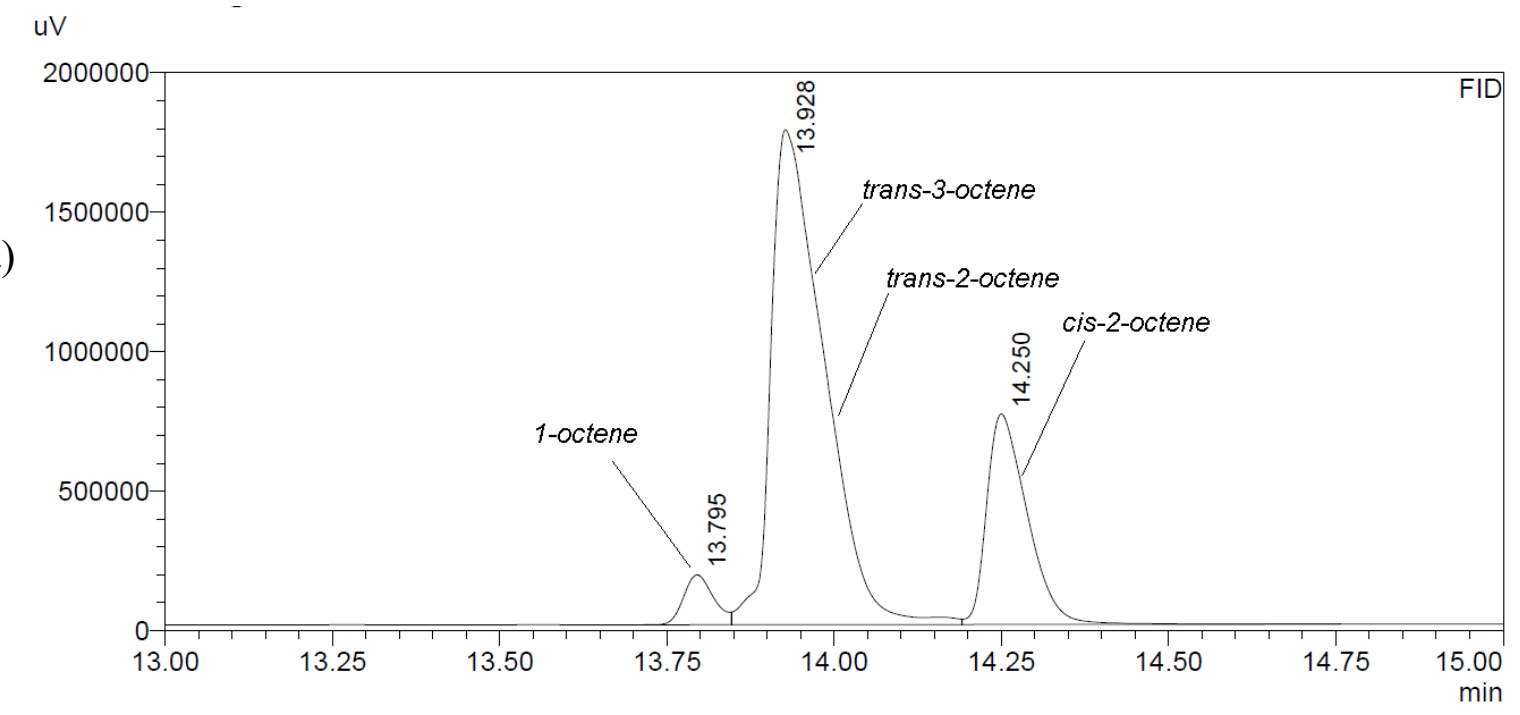

$\mathrm{uV}$

(b)

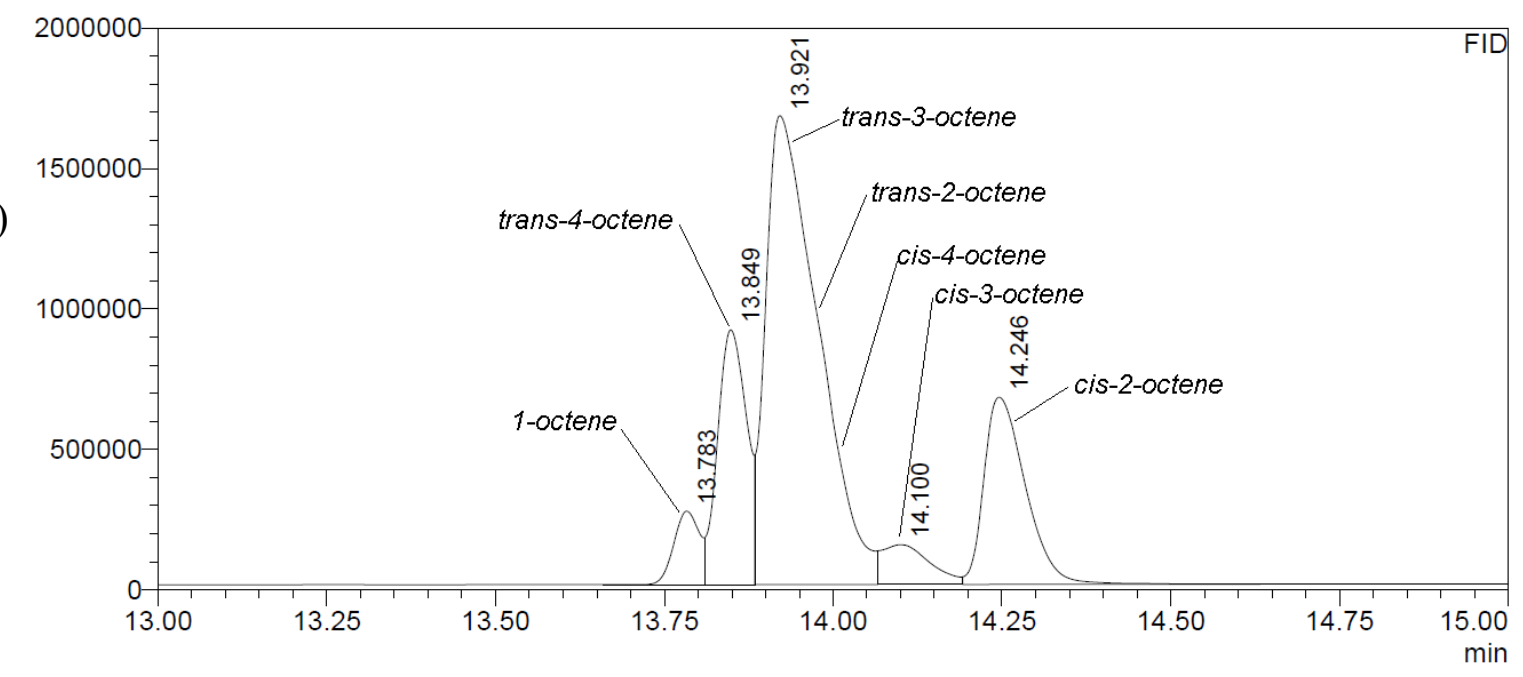

Figure S-35. GC traces of the catalytic isomerization of 1-octene to internal octenes by $\left[\left({ }^{\mathrm{Pr}} \mathrm{PONOP}\right) \mathrm{Mo}(\mathrm{CO})_{3} \mathrm{H}\right]^{+} \mathrm{BF}_{4}^{-}, \mathbf{3 b}$, in $\mathrm{CD}_{2} \mathrm{Cl}_{2}$ at $65^{\circ} \mathrm{C}$. [1-octene] $=0.22 \mathrm{M}$. $7 \%$ catalyst loading. (a) after $4.7 \mathrm{hrs}$, (b) after $40.7 \mathrm{hrs.} \mathrm{Shimadzu} \mathrm{GC-2010} \mathrm{with} \mathrm{Agilent} \mathrm{GS-GASPRO}$ PLOT column (30m x $0.32 \mathrm{~mm}$ ID), 100-250 ${ }^{\circ} \mathrm{C} @ 10^{\circ} / \mathrm{min}$, column flow: $2 \mathrm{~mL} / \mathrm{min}, 25: 1 \mathrm{split}$. 


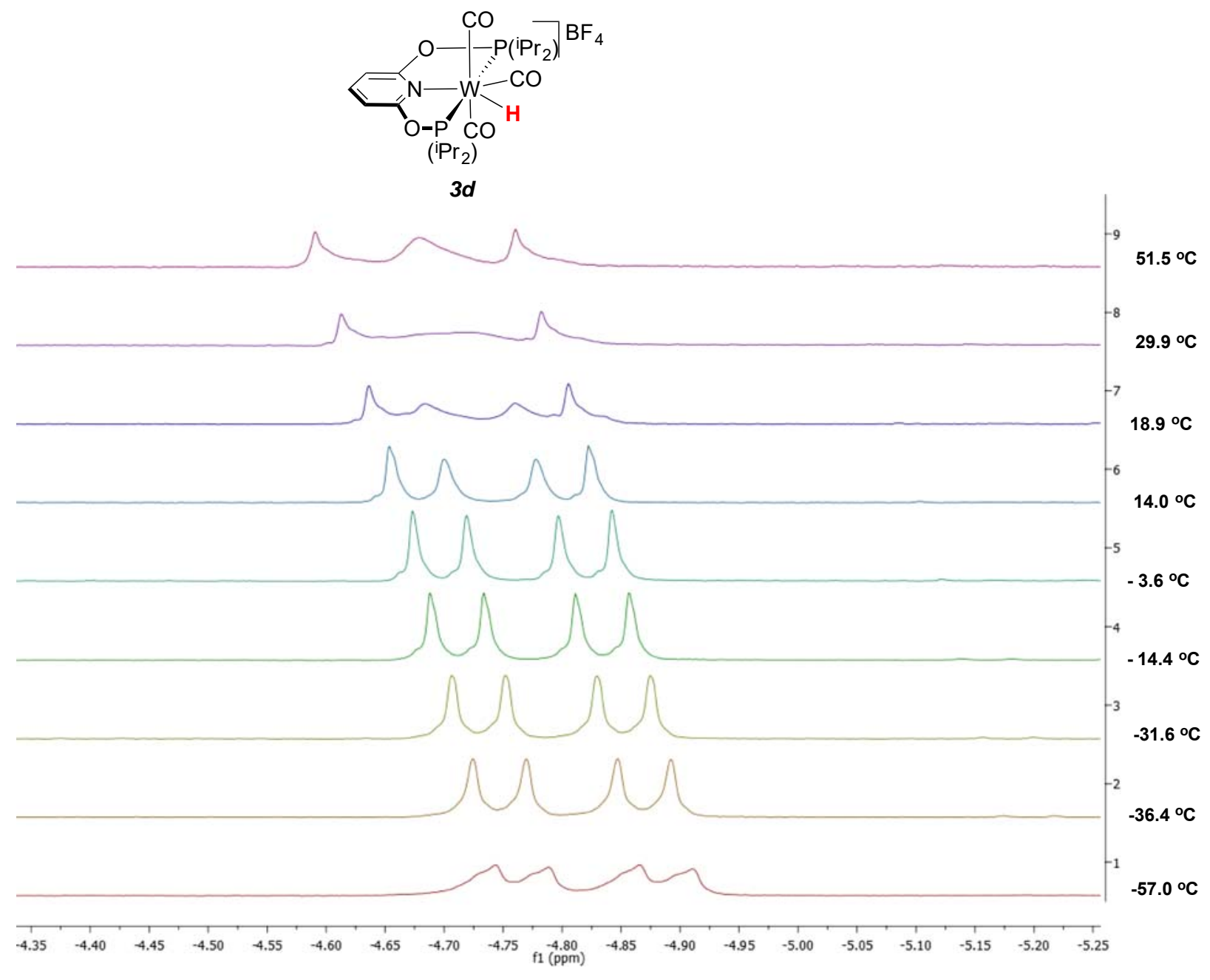

Figure S36. Variable Temperature ${ }^{1} \mathrm{H}$ NMR spectra in $\mathrm{CD}_{2} \mathrm{Cl}_{2}$ of complex 3d 
Figure S-37. Simulation of hydride resonance for complex $\mathbf{3 b}$, overlaid on actual spectra.

243 K

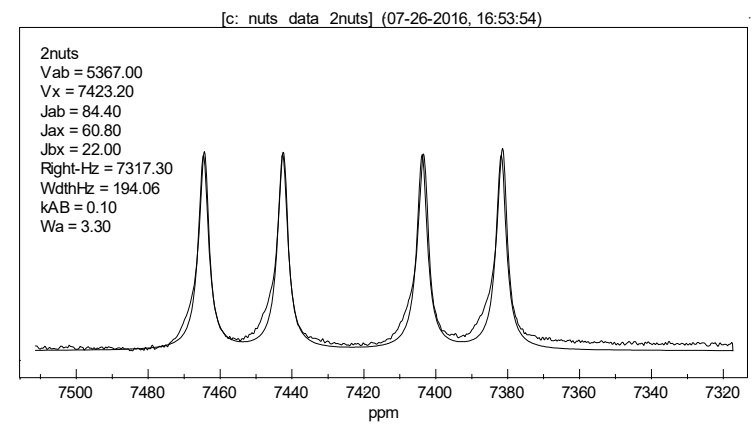

$253 \mathrm{~K}$

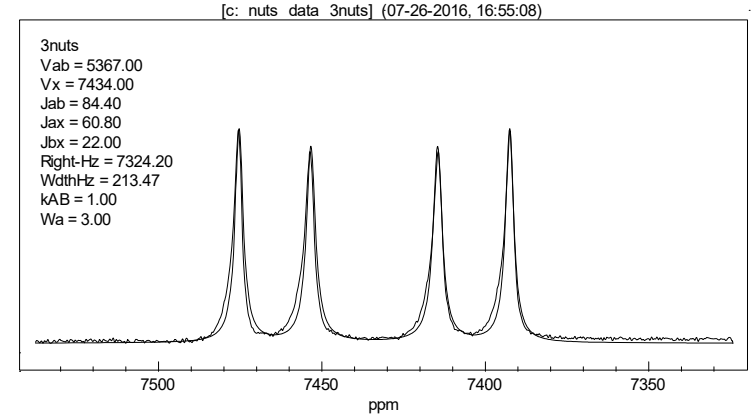

$263 \mathrm{~K}$

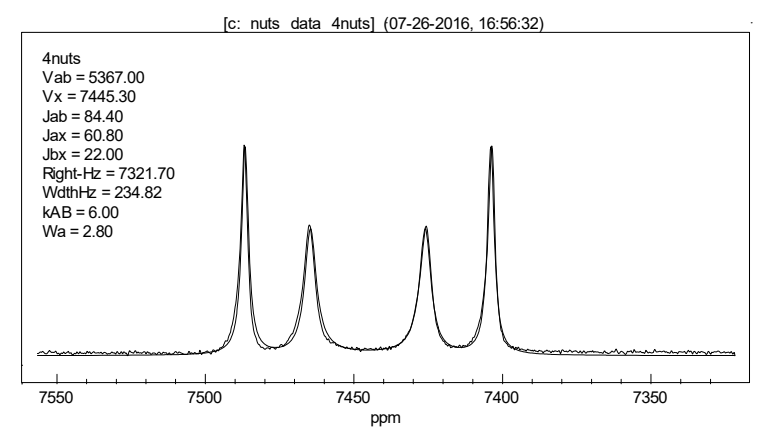

273 K

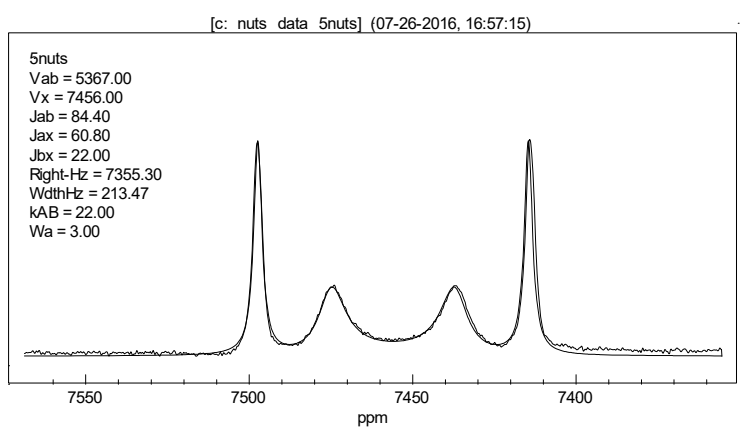

$283 \mathrm{~K}$

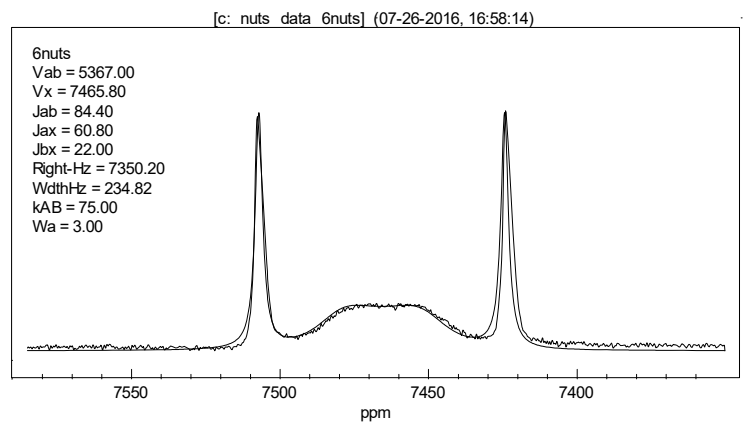

$293 \mathrm{~K}$

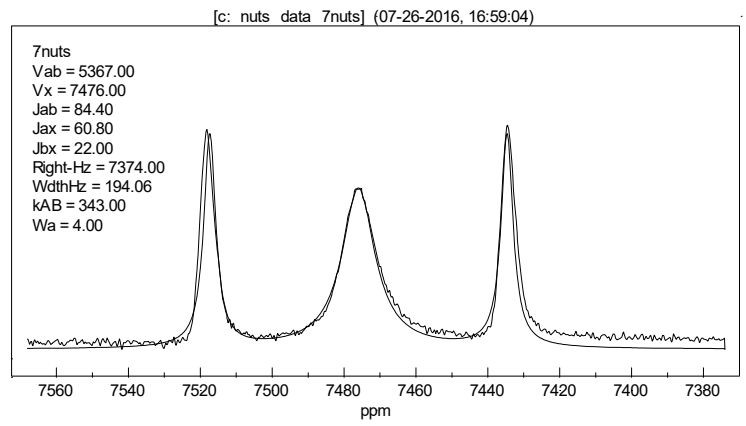

$303 \mathrm{~K}$

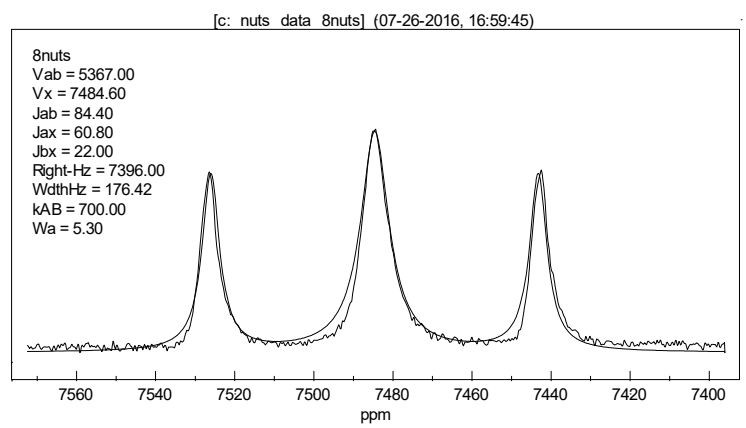

$313 \mathrm{~K}$

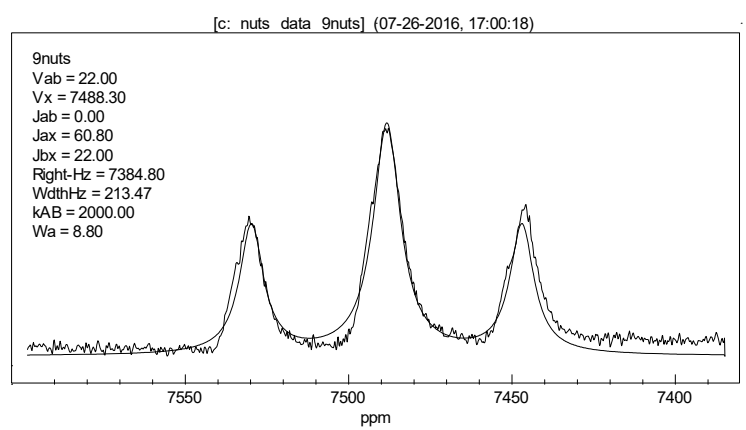


Figure S-38. Eyring plot for hydride fluxionality in complex $\mathbf{3 b}$.

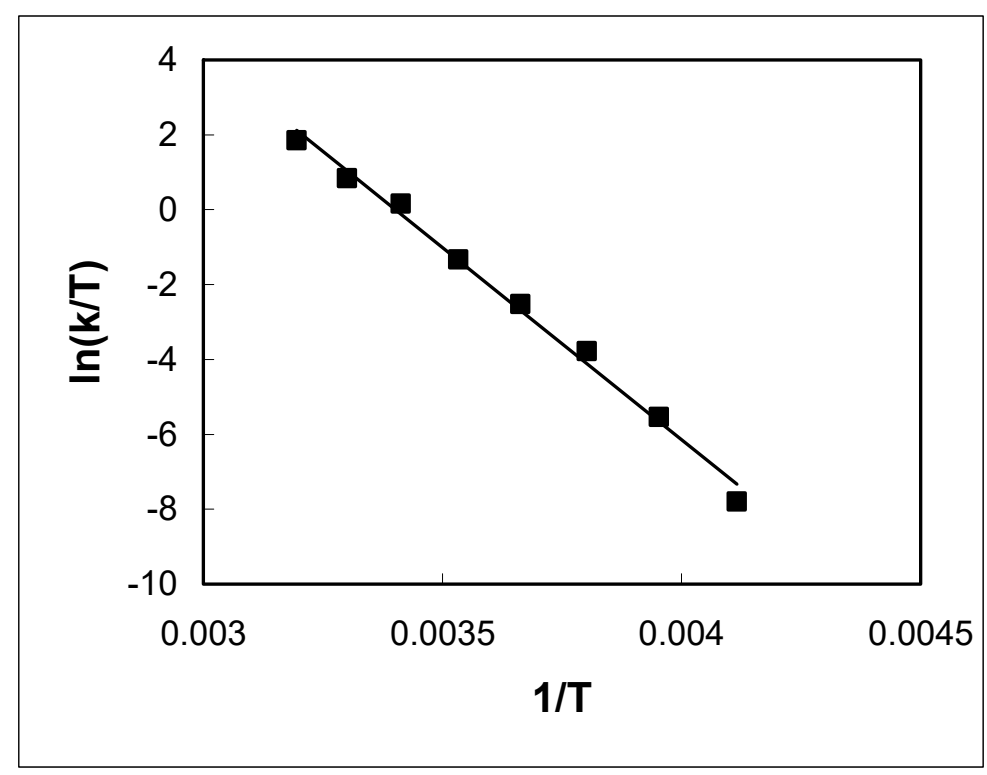

95\% confidence limits:

$\begin{aligned} \Delta \mathrm{H}^{\ddagger} & =20.41 \pm 0.62 \mathrm{kcal} / \mathrm{mol} \\ \Delta \mathrm{S}^{\ddagger} & =22.2 \pm 2.2 \mathrm{e} . \mathrm{u} . \\ \Delta \mathrm{G}^{\ddagger} & =14.0 \pm 1.2 \mathrm{kcal} / \mathrm{mol} @ 287 \mathrm{~K}\end{aligned}$


Table S-1. Crystal Data and Refinement Parameters for 2a-d.

\begin{tabular}{|c|c|c|c|c|}
\hline & $2 a$ & $2 b$ & $2 c$ & 2d \\
\hline cryst syst & $\begin{array}{l}\text { mono- } \\
\text { clinic }\end{array}$ & $\begin{array}{l}\text { mono- } \\
\text { clinic }\end{array}$ & $\begin{array}{l}\text { mono- } \\
\text { clinic }\end{array}$ & $\begin{array}{l}\text { ortho- } \\
\text { rhombic }\end{array}$ \\
\hline space group & $P 2_{1} / n$ & $P 2_{1} / n$ & $P 2_{1} / n$ & $P 2{ }_{1}{ }_{1} 2_{1}$ \\
\hline$a, \AA$ & $14.864(5)$ & $12.482(3)$ & $14.8572(16)$ & $10.7399(19)$ \\
\hline$b, \AA$ & $13.125(4)$ & $10.474(3)$ & $13.1404(14)$ & $11.323(2)$ \\
\hline$c, \AA$ & $15.573(5)$ & $19.450(5)$ & $15.5556(16)$ & $19.424(4)$ \\
\hline$\alpha, \operatorname{deg}$ & 90 & 90 & 90 & 90 \\
\hline$\beta, \operatorname{deg}$ & $17.983(6)$ & $108.701(4)$ & $117.8272(19)$ & 90 \\
\hline$\gamma, \operatorname{deg}$ & 90 & 90 & 90 & 90 \\
\hline Volume, $\AA^{3}$ & $682.8(15)$ & 2408.7(10) & $2685.7(5)$ & $2362.1(7)$ \\
\hline$Z$ & 4 & 4 & 4 & 4 \\
\hline 10. of data collecter & 98954 & 38510 & 99917 & 73539 \\
\hline $\begin{array}{c}\mathrm{R}_{1}, \mathrm{wR}_{2} \\
(\mathrm{I}>2 \sigma(\mathrm{I}))\end{array}$ & $\begin{array}{l}0.030 \\
0.0716\end{array}$ & $0.0580,0.1127$ & $.0245,0.0555]$ & $.0172,0.036 c$ \\
\hline $\begin{array}{l}\mathrm{R}_{1}, \mathrm{wR}_{2} \\
\text { (all data) }\end{array}$ & $\begin{array}{c}0.0450 \\
0.0798\end{array}$ & $0.0980,0.1263$ & $.0303,0.0574$ & $.0187,0.0363$ \\
\hline
\end{tabular}


Table S-2. Selected bond lengths ( $(\AA)$ and angles (deg).

$\begin{array}{lllll} & \begin{array}{l}\mathbf{2 a} \\ (\mathrm{M}=\mathrm{Mo}, \\ \left.\mathrm{R}={ }^{\mathrm{t}} \mathrm{Bu}\right)\end{array} & \begin{array}{l}\mathbf{2 b} \\ (\mathrm{M}=\mathrm{Mo}), \\ \left.\mathrm{R}={ }^{\mathrm{i}} \mathrm{Pr}\right)\end{array} & \begin{array}{l}\mathbf{2 c} \\ (\mathrm{M}=\mathrm{W}), \\ \left.\mathrm{R}={ }^{\mathrm{t}} \mathrm{Bu}\right)\end{array} & \begin{array}{l}\mathbf{2 d} \\ (\mathrm{M}=\mathrm{W}), \\ \mathrm{R}={ }^{\mathrm{i}} \mathrm{Pr}\end{array} \\ \mathrm{M}(1)-\mathrm{C}(22) / \mathrm{C}(1) & 2.0235(14) & 1.961(5) & 2.0161(17) & 1.969(2) \\ \mathrm{M}(1)-\mathrm{C}(23) / \mathrm{C}(2) & 1.9664(13) & 2.029(5) & 1.9696(17) & 2.019(2) \\ \mathrm{M}(1)-\mathrm{C}(24) / \mathrm{C}(3) & 2.0094(14) & 1.997(5) & 2.0073(18) & 2.016(2) \\ \mathrm{M}(1)-\mathrm{P}(1) & 2.4657(8) & 2.3957(13) & 2.4551(5) & 2.3801(6) \\ \mathrm{M}(1)-\mathrm{P}(2) & 2.4469(8) & 2.3904(14) & 2.4396(5) & 2.4038(6) \\ \mathrm{M}(1)-\mathrm{N}(1) & 2.2535(12) & 2.241(9) & 2.2501(13) & 2.2318(17) \\ \mathrm{P}(1)-\mathrm{M}(1)-\mathrm{P}(2) & 150.023(16) & 152.93(4) & 150.113(14) & 152.62(2) \\ \mathrm{N}(1)-\mathrm{M}(1)-\mathrm{P}(1) & 75.55(2) & 76.42(10) & 75.58(3) & 76.57(5) \\ \mathrm{N}(1)-\mathrm{M}(1)-\mathrm{P}(2) & 75.50(2) & 76.54(10) & 75.52(3) & 76.17(5) \\ \mathrm{N}(1)-\mathrm{M}(1)-\mathrm{C}(22) / \mathrm{C}(1) & 90.18(4) & 178.13(18) & 91.11(6) & 176.42(8) \\ \mathrm{N}(1)-\mathrm{M}(1)-\mathrm{C}(23) / \mathrm{C}(2) & 175.78(4) & 93.94(17) & 175.67(6) & 97.42(8) \\ \mathrm{N}(1)-\mathrm{M}(1)-\mathrm{C}(24) / \mathrm{C}(3) & 106.96(4) & 99.86(18) & 106.97(6) & 96.43(7) \\ \mathrm{C}(22) / \mathrm{C}(2)-\mathrm{M}(1)-\mathrm{C}(24) / \mathrm{C}(3) & 162.87(5) & 166.1(2) & 161.91(7) & 166.14(9) \\ & & & & \end{array}$


CRYSTAL STRUCTURE REPORT

$\mathrm{C}_{24} \mathrm{H}_{39} \mathrm{Mo} \mathrm{N} \mathrm{O}_{5} \mathrm{P}_{2}$

Report prepared for:

Dr. R. Castro-Rodrigo, Prof. W. Jones

October 15, 2013

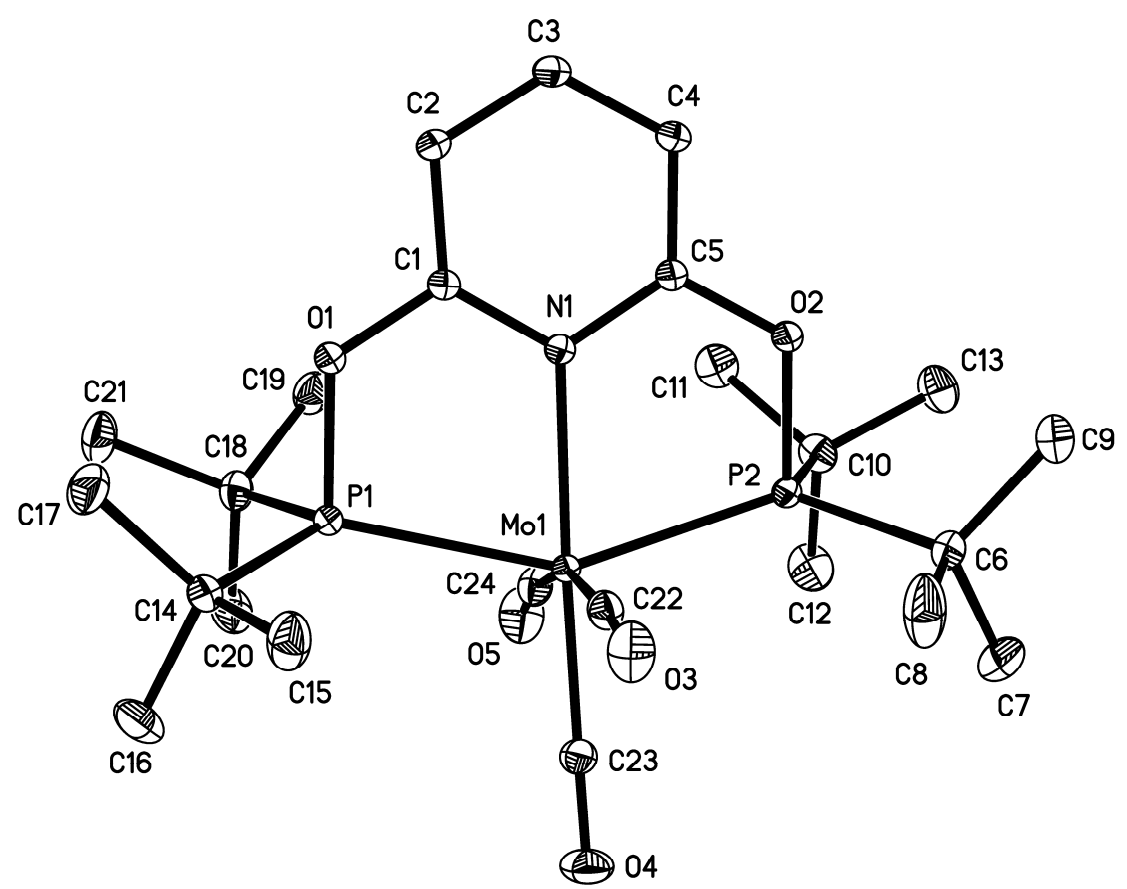

Ruth Castro-Rodrigo

X-ray Crystallographic Facility

Department of Chemistry, University of Rochester

120 Trustee Road

Rochester, NY 14627 
Data collection

A crystal $\left(0.300 \times 0.300 \times 0.120 \mathrm{~mm}^{3}\right)$ was placed onto the tip of a $0.1 \mathrm{~mm}$ diameter glass capillary tube or fiber and mounted on a Bruker SMART APEX II CCD platform diffractometer for a data collection at $100.0(5) \mathrm{K} .{ }^{1}$ A preliminary set of cell constants and an orientation matrix were calculated from reflections harvested from three orthogonal wedges of reciprocal space. The full data collection was carried out using MoK $\alpha$ radiation (graphite monochromator) with a frame time of 15 seconds and a detector distance of $4.02 \mathrm{~cm}$. A randomly oriented region of reciprocal space was surveyed: six major sections of frames were collected with $0.50^{\circ}$ steps in $\omega$ at six different $\phi$ settings and a detector position of $-38^{\circ}$ in $2 \theta$. The intensity data were corrected for absorption. ${ }^{2}$ Final cell constants were calculated from the xyz centroids of 3765 strong reflections from the actual data collection after integration. ${ }^{3}$ See Table S1 for additional crystal and refinement information.

Structure solution and refinement

The structure was solved using SHELXS-2013 ${ }^{4}$ and refined using SHELXL-2013. ${ }^{5}$ The space group $P 2{ }_{1} / n$ was determined based on systematic absences. A direct-methods solution was calculated which provided most nonhydrogen atoms from the E-map. Full-matrix least squares / difference Fourier cycles were performed which located the remaining non-hydrogen atoms. All non-hydrogen atoms were refined with anisotropic displacement parameters. All hydrogen atoms were placed in ideal positions and refined as riding atoms with relative isotropic displacement parameters. The final full matrix least squares refinement converged to $R 1=0.0309\left(F^{2}, I>2 \sigma(I)\right)$ and $w R 2=0.0798\left(F^{2}\right.$, all data $)$.

\section{Structure description}

The structure is the one suggested. The asymmetric unit contains one molybdenum molecule in a general position.

Unless noted otherwise all structural diagrams containing thermal displacement ellipsoids are drawn at the $50 \%$ probability level.

Data collection, structure solution, and structure refinement were conducted at the X-ray Crystallographic Facility, B51 Hutchison Hall, Department of Chemistry, University of Rochester. All publications arising from this report MUST either 1) include Ruth Castro-Rodrigo as a coauthor or 2) acknowledge Ruth Castro-Rodrigo and the X-ray Crystallographic Facility of the Department of Chemistry at the University of Rochester. 
1 APEX2, version 2013.2-0; Bruker AXS: Madison, WI, 2013.

2 Sheldrick, G. M. SADABS, version 2012/1; University of Göttingen: Göttingen, Germany, 2012.

3 SAINT, version 8.27B; Bruker AXS: Madison, WI, 2013.

4 Sheldrick, G. M. SHELXS-2013/1; University of Göttingen: Göttingen, Germany, 2013.

5 Sheldrick, G. M. SHELXL-2013/4; University of Göttingen: Göttingen, Germany, 2013.

Some equations of interest:

$$
\begin{gathered}
R_{\mathrm{int}}=\Sigma\left|F_{\mathrm{o}}^{2}-<F_{\mathrm{o}}^{2}>\right| / \Sigma\left|F_{\mathrm{o}}{ }^{2}\right| \\
R 1=\Sigma|| F_{\mathrm{o}}|-| F_{\mathrm{c}} \| / \Sigma\left|F_{\mathrm{o}}\right| \\
w R 2=\left[\Sigma\left[w\left(F_{\mathrm{o}}{ }^{2}-F_{\mathrm{c}}{ }^{2}\right)^{2}\right] / \Sigma\left[w\left(F_{\mathrm{o}}{ }^{2}\right)^{2}\right]\right]^{1 / 2} \\
\text { where } w=1 /\left[\sigma^{2}\left(F_{\mathrm{o}}{ }^{2}\right)+(a P)^{2}+b P\right] \text { and } \\
P=1 / 3 \max \left(0, F_{\mathrm{o}}{ }^{2}\right)+2 / 3 F_{\mathrm{c}}{ }^{2} \\
\mathrm{GOF}=S=\left[\Sigma\left[w\left(F_{\mathrm{o}}{ }^{2}-F_{\mathrm{c}}{ }^{2}\right)^{2}\right] /(m-n)\right]^{1 / 2}
\end{gathered}
$$

where $m=$ number of reflections and $n=$ number of parameters

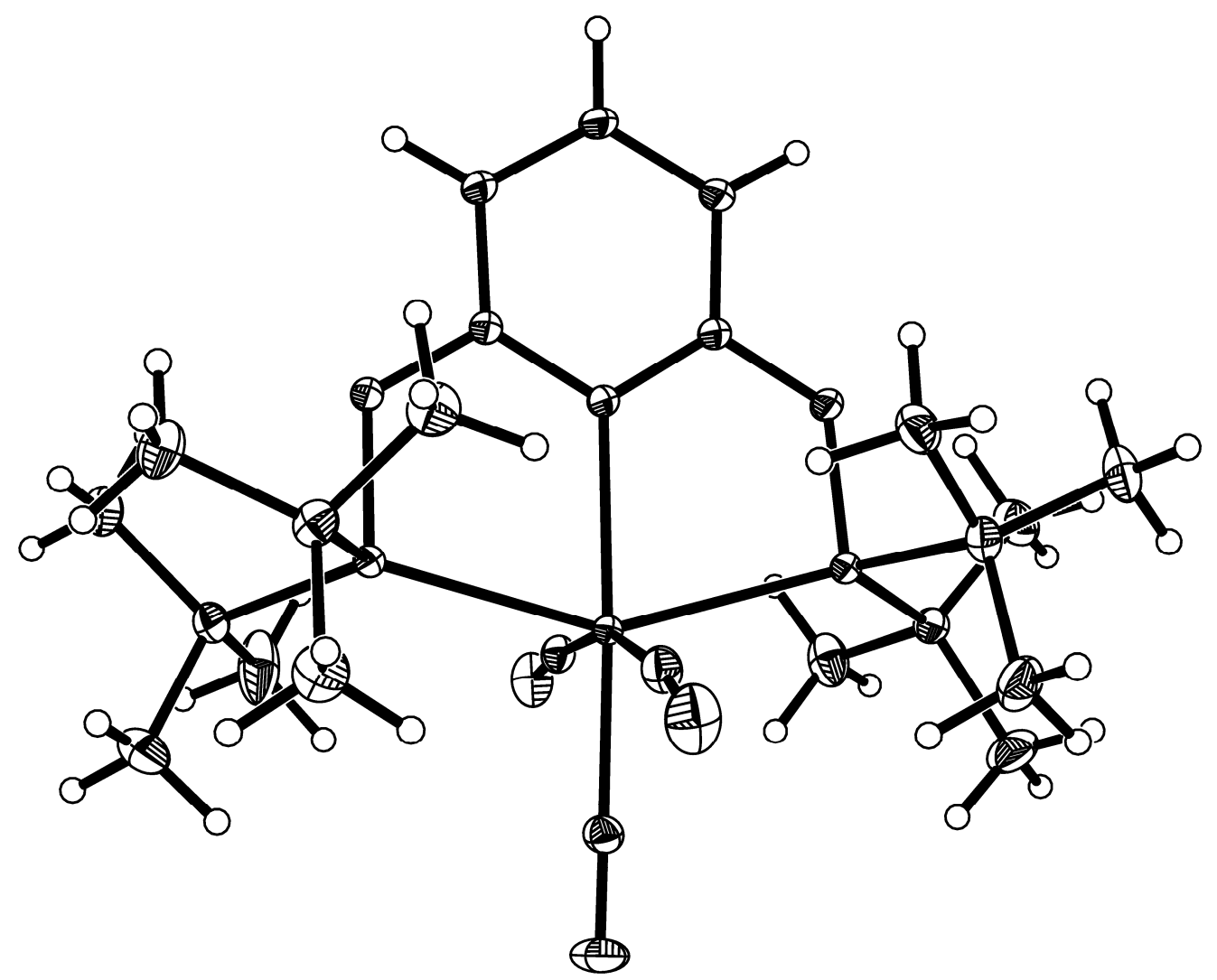


Table S-3. Crystal data and structure refinement for jonrcr01. (2a)

\begin{tabular}{|c|c|c|}
\hline Identification code & \multicolumn{2}{|l|}{ jonrcr01 } \\
\hline Empirical formula & \multicolumn{2}{|c|}{$\mathrm{C}_{24} \mathrm{H}_{39} \mathrm{Mo} \mathrm{N} \mathrm{O} \mathrm{P}_{2}$} \\
\hline Formula weight & \multicolumn{2}{|l|}{579.44} \\
\hline Temperature & \multicolumn{2}{|l|}{$100.0(5) \mathrm{K}$} \\
\hline Wavelength & \multicolumn{2}{|l|}{$0.71073 \AA$} \\
\hline Crystal system & \multicolumn{2}{|l|}{ monoclinic } \\
\hline Space group & \multicolumn{2}{|l|}{$P 2_{1} / n$} \\
\hline \multirow[t]{3}{*}{ Unit cell dimensions } & $a=14.864(5) \AA$ & $\alpha=90^{\circ}$ \\
\hline & $b=13.125(4) \AA$ & $\beta=117.983(6)^{\circ}$ \\
\hline & $c=15.573(5) \AA$ & $\gamma=90^{\circ}$ \\
\hline Volume & \multicolumn{2}{|l|}{$2682.8(15) \AA^{3}$} \\
\hline$Z$ & \multicolumn{2}{|l|}{4} \\
\hline Density (calculated) & \multicolumn{2}{|l|}{$1.435 \mathrm{Mg} / \mathrm{m}^{3}$} \\
\hline Absorption coefficient & \multicolumn{2}{|l|}{$0.641 \mathrm{~mm}^{-1}$} \\
\hline$F(000)$ & \multicolumn{2}{|l|}{1208} \\
\hline Crystal color, morphology & \multicolumn{2}{|l|}{ yellow, block } \\
\hline Crystal size & \multicolumn{2}{|c|}{$0.300 \times 0.300 \times 0.120 \mathrm{~mm}^{3}$} \\
\hline Theta range for data collection & \multicolumn{2}{|l|}{1.563 to $38.692^{\circ}$} \\
\hline Index ranges & \multicolumn{2}{|c|}{$-26 \leq h \leq 26,-23 \leq k \leq 22,-27 \leq l \leq 27$} \\
\hline Reflections collected & \multicolumn{2}{|l|}{98954} \\
\hline Independent reflections & \multicolumn{2}{|c|}{$14879[R($ int $)=0.0479]$} \\
\hline Observed reflections & \multicolumn{2}{|l|}{12015} \\
\hline Completeness to theta $=37.785^{\circ}$ & \multicolumn{2}{|l|}{$100.0 \%$} \\
\hline Absorption correction & \multicolumn{2}{|l|}{ Multi-scan } \\
\hline Max. and min. transmission & \multicolumn{2}{|l|}{0.7476 and 0.6370} \\
\hline Refinement method & \multicolumn{2}{|c|}{ Full-matrix least-squares on $F^{2}$} \\
\hline Data / restraints / parameters & \multicolumn{2}{|l|}{$14879 / 0 / 310$} \\
\hline Goodness-of-fit on $F^{2}$ & \multicolumn{2}{|l|}{1.004} \\
\hline Final $R$ indices $[I>2 \operatorname{sigma}(I)]$ & \multicolumn{2}{|c|}{$R 1=0.0309, w R 2=0.0716$} \\
\hline$R$ indices (all data) & \multicolumn{2}{|c|}{$R 1=0.0450, w R 2=0.0798$} \\
\hline Largest diff. peak and hole & \multicolumn{2}{|c|}{1.420 and -0.963 e..$\AA^{-3}$} \\
\hline
\end{tabular}


Table S-4. Atomic coordinates (x $\left.10^{4}\right)$ and equivalent isotropic displacement parameters $\left(\AA^{2} \times 10^{3}\right)$ for jonrcr01 (2a). $U_{e q}$ is defined as one third of the trace of the orthogonalized $U_{i j}$ tensor.

\begin{tabular}{|c|c|c|c|c|}
\hline & $\mathrm{x}$ & $\mathrm{y}$ & $\mathrm{z}$ & $\mathrm{U}_{\mathrm{eq}}$ \\
\hline Mo1 & $9116(1)$ & $8897(1)$ & $1887(1)$ & $10(1)$ \\
\hline P1 & $9604(1)$ & $7085(1)$ & 2194(1) & $12(1)$ \\
\hline $\mathrm{P} 2$ & $9595(1)$ & 10692(1) & 1979(1) & 11(1) \\
\hline $\mathrm{O} 1$ & 10767(1) & 7097(1) & $2237(1)$ & $14(1)$ \\
\hline $\mathrm{O} 2$ & 10741(1) & 10617(1) & 1992(1) & $13(1)$ \\
\hline $\mathrm{O} 3$ & $8284(1)$ & $8752(1)$ & $-396(1)$ & $23(1)$ \\
\hline $\mathrm{O} 4$ & $6810(1)$ & $9008(1)$ & $1344(1)$ & $24(1)$ \\
\hline O5 & $8964(1)$ & $9059(1)$ & $3836(1)$ & $28(1)$ \\
\hline N1 & $10755(1)$ & $8864(1)$ & $2189(1)$ & 11(1) \\
\hline $\mathrm{C} 1$ & $11267(1)$ & $7979(1)$ & $2330(1)$ & $12(1)$ \\
\hline $\mathrm{C} 2$ & $12289(1)$ & $7927(1)$ & $2560(1)$ & $15(1)$ \\
\hline $\mathrm{C} 3$ & 12786(1) & $8833(1)$ & 2601(1) & $16(1)$ \\
\hline $\mathrm{C} 4$ & $12267(1)$ & $9750(1)$ & 2404(1) & $14(1)$ \\
\hline $\mathrm{C} 5$ & $11252(1)$ & $9728(1)$ & $2200(1)$ & $11(1)$ \\
\hline C6 & 8981(1) & $11646(1)$ & $959(1)$ & $17(1)$ \\
\hline $\mathrm{C} 7$ & $8151(1)$ & $12228(1)$ & $1090(1)$ & $31(1)$ \\
\hline $\mathrm{C} 8$ & $8468(2)$ & $11078(1)$ & $4(1)$ & $35(1)$ \\
\hline C9 & 9732(1) & 12399(1) & $889(1)$ & $24(1)$ \\
\hline $\mathrm{C} 10$ & 10053(1) & 11386(1) & $3176(1)$ & $16(1)$ \\
\hline C11 & 10911(1) & $10720(1)$ & $3948(1)$ & $21(1)$ \\
\hline $\mathrm{C} 12$ & $9172(1)$ & $11490(1)$ & $3425(1)$ & $25(1)$ \\
\hline $\mathrm{C} 13$ & 10526(1) & $12438(1)$ & $3236(1)$ & $23(1)$ \\
\hline C14 & $9024(1)$ & $6019(1)$ & $1288(1)$ & $15(1)$ \\
\hline C15 & $8562(1)$ & $6468(1)$ & $265(1)$ & $24(1)$ \\
\hline $\mathrm{C} 16$ & $8158(1)$ & $5540(1)$ & $1423(1)$ & $28(1)$ \\
\hline $\mathrm{C} 17$ & 9789(1) & $5200(1)$ & $1348(1)$ & $24(1)$ \\
\hline C18 & 10033(1) & $6561(1)$ & $3459(1)$ & $17(1)$ \\
\hline C19 & 10872(1) & 7294(1) & $4155(1)$ & $21(1)$ \\
\hline $\mathrm{C} 20$ & $9137(1)$ & $6551(1)$ & $3692(1)$ & $24(1)$ \\
\hline $\mathrm{C} 21$ & 10518(1) & $5496(1)$ & $3659(1)$ & $24(1)$ \\
\hline $\mathrm{C} 22$ & $8651(1)$ & 8801(1) & $443(1)$ & $14(1)$ \\
\hline $\mathrm{C} 23$ & $7660(1)$ & $8954(1)$ & $1525(1)$ & $15(1)$ \\
\hline C24 & $9128(1)$ & 8997(1) & $3180(1)$ & $17(1)$ \\
\hline
\end{tabular}


Table S-5. Bond lengths $[\AA]$ and angles $\left[^{\circ}\right]$ for jonrcr01 (2a).

\begin{tabular}{|c|c|c|c|}
\hline $\operatorname{Mo}(1)-C(23)$ & $1.9664(13)$ & $\mathrm{C}(9)-\mathrm{H}(9 \mathrm{~B})$ & 0.9800 \\
\hline $\operatorname{Mo}(1)-C(24)$ & $2.0094(14)$ & $\mathrm{C}(9)-\mathrm{H}(9 \mathrm{C})$ & 0.9800 \\
\hline $\operatorname{Mo}(1)-C(22)$ & $2.0235(14)$ & $C(10)-C(13)$ & $1.5323(18)$ \\
\hline $\mathrm{Mo}(1)-\mathrm{N}(1)$ & $2.2535(12)$ & $C(10)-C(12)$ & $1.5349(18)$ \\
\hline $\mathrm{Mo}(1)-\mathrm{P}(2)$ & $2.4469(8)$ & $\mathrm{C}(10)-\mathrm{C}(11)$ & $1.5481(19)$ \\
\hline $\mathrm{Mo}(1)-\mathrm{P}(1)$ & $2.4657(8)$ & $\mathrm{C}(11)-\mathrm{H}(11 \mathrm{~A})$ & 0.9800 \\
\hline $\mathrm{P}(1)-\mathrm{O}(1)$ & $1.6981(10)$ & $\mathrm{C}(11)-\mathrm{H}(11 \mathrm{~B})$ & 0.9800 \\
\hline$P(1)-C(14)$ & $1.8852(13)$ & $\mathrm{C}(11)-\mathrm{H}(11 \mathrm{C})$ & 0.9800 \\
\hline $\mathrm{P}(1)-\mathrm{C}(18)$ & $1.8920(14)$ & $\mathrm{C}(12)-\mathrm{H}(12 \mathrm{~A})$ & 0.9800 \\
\hline $\mathrm{P}(2)-\mathrm{O}(2)$ & $1.6968(10)$ & $\mathrm{C}(12)-\mathrm{H}(12 \mathrm{~B})$ & 0.9800 \\
\hline $\mathrm{P}(2)-\mathrm{C}(6)$ & $1.8874(13)$ & $\mathrm{C}(12)-\mathrm{H}(12 \mathrm{C})$ & 0.9800 \\
\hline $\mathrm{P}(2)-\mathrm{C}(10)$ & $1.8921(13)$ & $\mathrm{C}(13)-\mathrm{H}(13 \mathrm{~A})$ & 0.9800 \\
\hline $\mathrm{O}(1)-\mathrm{C}(1)$ & $1.3467(14)$ & $\mathrm{C}(13)-\mathrm{H}(13 \mathrm{~B})$ & 0.9800 \\
\hline $\mathrm{O}(2)-\mathrm{C}(5)$ & $1.3456(14)$ & $\mathrm{C}(13)-\mathrm{H}(13 \mathrm{C})$ & 0.9800 \\
\hline $\mathrm{O}(3)-\mathrm{C}(22)$ & $1.1575(15)$ & $\mathrm{C}(14)-\mathrm{C}(15)$ & $1.5263(19)$ \\
\hline $\mathrm{O}(4)-\mathrm{C}(23)$ & $1.1598(15)$ & $\mathrm{C}(14)-\mathrm{C}(16)$ & $1.5341(18)$ \\
\hline $\mathrm{O}(5)-\mathrm{C}(24)$ & $1.1594(16)$ & $C(14)-C(17)$ & $1.5351(17)$ \\
\hline $\mathrm{N}(1)-\mathrm{C}(5)$ & $1.3496(14)$ & $\mathrm{C}(15)-\mathrm{H}(15 \mathrm{~A})$ & 0.9800 \\
\hline $\mathrm{N}(1)-\mathrm{C}(1)$ & $1.3495(14)$ & $\mathrm{C}(15)-\mathrm{H}(15 \mathrm{~B})$ & 0.9800 \\
\hline$C(1)-C(2)$ & $1.3891(15)$ & $\mathrm{C}(15)-\mathrm{H}(15 \mathrm{C})$ & 0.9800 \\
\hline $\mathrm{C}(2)-\mathrm{C}(3)$ & $1.3843(16)$ & $\mathrm{C}(16)-\mathrm{H}(16 \mathrm{~A})$ & 0.9800 \\
\hline $\mathrm{C}(2)-\mathrm{H}(2 \mathrm{~A})$ & 0.9500 & $\mathrm{C}(16)-\mathrm{H}(16 \mathrm{~B})$ & 0.9800 \\
\hline$C(3)-C(4)$ & $1.3848(16)$ & $\mathrm{C}(16)-\mathrm{H}(16 \mathrm{C})$ & 0.9800 \\
\hline $\mathrm{C}(3)-\mathrm{H}(3 \mathrm{~A})$ & 0.9500 & $\mathrm{C}(17)-\mathrm{H}(17 \mathrm{~A})$ & 0.9800 \\
\hline $\mathrm{C}(4)-\mathrm{C}(5)$ & $1.3887(15)$ & $\mathrm{C}(17)-\mathrm{H}(17 \mathrm{~B})$ & 0.9800 \\
\hline $\mathrm{C}(4)-\mathrm{H}(4 \mathrm{~A})$ & 0.9500 & $\mathrm{C}(17)-\mathrm{H}(17 \mathrm{C})$ & 0.9800 \\
\hline$C(6)-C(8)$ & $1.512(2)$ & $\mathrm{C}(18)-\mathrm{C}(21)$ & $1.5370(18)$ \\
\hline$C(6)-C(9)$ & $1.5326(18)$ & $C(18)-C(20)$ & $1.5350(19)$ \\
\hline$C(6)-C(7)$ & $1.544(2)$ & $C(18)-C(19)$ & $1.5445(18)$ \\
\hline $\mathrm{C}(7)-\mathrm{H}(7 \mathrm{~A})$ & 0.9800 & $\mathrm{C}(19)-\mathrm{H}(19 \mathrm{~A})$ & 0.9800 \\
\hline $\mathrm{C}(7)-\mathrm{H}(7 \mathrm{~B})$ & 0.9800 & $\mathrm{C}(19)-\mathrm{H}(19 \mathrm{~B})$ & 0.9800 \\
\hline $\mathrm{C}(7)-\mathrm{H}(7 \mathrm{C})$ & 0.9800 & $\mathrm{C}(19)-\mathrm{H}(19 \mathrm{C})$ & 0.9800 \\
\hline $\mathrm{C}(8)-\mathrm{H}(8 \mathrm{~A})$ & 0.9800 & $\mathrm{C}(20)-\mathrm{H}(20 \mathrm{~A})$ & 0.9800 \\
\hline $\mathrm{C}(8)-\mathrm{H}(8 \mathrm{~B})$ & 0.9800 & $\mathrm{C}(20)-\mathrm{H}(20 \mathrm{~B})$ & 0.9800 \\
\hline $\mathrm{C}(8)-\mathrm{H}(8 \mathrm{C})$ & 0.9800 & $\mathrm{C}(20)-\mathrm{H}(20 \mathrm{C})$ & 0.9800 \\
\hline $\mathrm{C}(9)-\mathrm{H}(9 \mathrm{~A})$ & 0.9800 & $\mathrm{C}(21)-\mathrm{H}(21 \mathrm{~A})$ & 0.9800 \\
\hline
\end{tabular}




\begin{tabular}{|c|c|c|c|}
\hline $\mathrm{C}(21)-\mathrm{H}(21 \mathrm{~B})$ & 0.9800 & $C(3)-C(2)-C(1)$ & $117.74(10)$ \\
\hline \multirow[t]{2}{*}{$\mathrm{C}(21)-\mathrm{H}(21 \mathrm{C})$} & 0.9800 & $\mathrm{C}(3)-\mathrm{C}(2)-\mathrm{H}(2 \mathrm{~A})$ & 121.1 \\
\hline & & $\mathrm{C}(1)-\mathrm{C}(2)-\mathrm{H}(2 \mathrm{~A})$ & 121.1 \\
\hline $\mathrm{C}(23)-\mathrm{Mo}(1)-\mathrm{C}(24)$ & $77.04(5)$ & $\mathrm{C}(4)-\mathrm{C}(3)-\mathrm{C}(2)$ & $120.45(10)$ \\
\hline $\mathrm{C}(23)-\mathrm{Mo}(1)-\mathrm{C}(22)$ & $85.84(5)$ & $\mathrm{C}(4)-\mathrm{C}(3)-\mathrm{H}(3 \mathrm{~A})$ & 119.8 \\
\hline $\mathrm{C}(24)-\mathrm{Mo}(1)-\mathrm{C}(22)$ & $162.87(5)$ & $\mathrm{C}(2)-\mathrm{C}(3)-\mathrm{H}(3 \mathrm{~A})$ & 119.8 \\
\hline $\mathrm{C}(23)-\mathrm{Mo}(1)-\mathrm{N}(1)$ & $175.78(4)$ & $C(3)-C(4)-C(5)$ & $117.66(10)$ \\
\hline $\mathrm{C}(24)-\mathrm{Mo}(1)-\mathrm{N}(1)$ & $106.96(4)$ & $\mathrm{C}(3)-\mathrm{C}(4)-\mathrm{H}(4 \mathrm{~A})$ & 121.2 \\
\hline $\mathrm{C}(22)-\mathrm{Mo}(1)-\mathrm{N}(1)$ & $90.18(4)$ & $\mathrm{C}(5)-\mathrm{C}(4)-\mathrm{H}(4 \mathrm{~A})$ & 121.2 \\
\hline $\mathrm{C}(23)-\mathrm{Mo}(1)-\mathrm{P}(2)$ & $103.35(3)$ & $\mathrm{O}(2)-\mathrm{C}(5)-\mathrm{N}(1)$ & $118.76(9)$ \\
\hline $\mathrm{C}(24)-\mathrm{Mo}(1)-\mathrm{P}(2)$ & $90.76(4)$ & $\mathrm{O}(2)-\mathrm{C}(5)-\mathrm{C}(4)$ & $117.80(9)$ \\
\hline $\mathrm{C}(22)-\mathrm{Mo}(1)-\mathrm{P}(2)$ & $93.62(3)$ & $\mathrm{N}(1)-\mathrm{C}(5)-\mathrm{C}(4)$ & $123.44(10)$ \\
\hline $\mathrm{N}(1)-\mathrm{Mo}(1)-\mathrm{P}(2)$ & $75.50(2)$ & $\mathrm{C}(8)-\mathrm{C}(6)-\mathrm{C}(9)$ & $107.20(12)$ \\
\hline $\mathrm{C}(23)-\mathrm{Mo}(1)-\mathrm{P}(1)$ & $106.07(3)$ & $C(8)-C(6)-C(7)$ & $107.97(13)$ \\
\hline $\mathrm{C}(24)-\mathrm{Mo}(1)-\mathrm{P}(1)$ & $90.29(3)$ & $C(9)-C(6)-C(7)$ & $110.16(12)$ \\
\hline $\mathrm{C}(22)-\mathrm{Mo}(1)-\mathrm{P}(1)$ & $94.11(3)$ & $\mathrm{C}(8)-\mathrm{C}(6)-\mathrm{P}(2)$ & $108.81(9)$ \\
\hline $\mathrm{N}(1)-\mathrm{Mo}(1)-\mathrm{P}(1)$ & $75.55(2)$ & $\mathrm{C}(9)-\mathrm{C}(6)-\mathrm{P}(2)$ & 113.99(9) \\
\hline $\mathrm{P}(2)-\mathrm{Mo}(1)-\mathrm{P}(1)$ & $150.023(16)$ & $C(7)-C(6)-P(2)$ & $108.55(9)$ \\
\hline $\mathrm{O}(1)-\mathrm{P}(1)-\mathrm{C}(14)$ & $97.76(5)$ & $\mathrm{C}(6)-\mathrm{C}(7)-\mathrm{H}(7 \mathrm{~A})$ & 109.5 \\
\hline $\mathrm{O}(1)-\mathrm{P}(1)-\mathrm{C}(18)$ & $97.07(5)$ & $\mathrm{C}(6)-\mathrm{C}(7)-\mathrm{H}(7 \mathrm{~B})$ & 109.5 \\
\hline $\mathrm{C}(14)-\mathrm{P}(1)-\mathrm{C}(18)$ & 108.77(6) & $\mathrm{H}(7 \mathrm{~A})-\mathrm{C}(7)-\mathrm{H}(7 \mathrm{~B})$ & 109.5 \\
\hline $\mathrm{O}(1)-\mathrm{P}(1)-\mathrm{Mo}(1)$ & $101.57(3)$ & $\mathrm{C}(6)-\mathrm{C}(7)-\mathrm{H}(7 \mathrm{C})$ & 109.5 \\
\hline $\mathrm{C}(14)-\mathrm{P}(1)-\mathrm{Mo}(1)$ & $125.53(4)$ & $\mathrm{H}(7 \mathrm{~A})-\mathrm{C}(7)-\mathrm{H}(7 \mathrm{C})$ & 109.5 \\
\hline $\mathrm{C}(18)-\mathrm{P}(1)-\mathrm{Mo}(1)$ & $118.50(4)$ & $\mathrm{H}(7 \mathrm{~B})-\mathrm{C}(7)-\mathrm{H}(7 \mathrm{C})$ & 109.5 \\
\hline $\mathrm{O}(2)-\mathrm{P}(2)-\mathrm{C}(6)$ & $97.80(5)$ & $\mathrm{C}(6)-\mathrm{C}(8)-\mathrm{H}(8 \mathrm{~A})$ & 109.5 \\
\hline $\mathrm{O}(2)-\mathrm{P}(2)-\mathrm{C}(10)$ & $97.01(5)$ & $\mathrm{C}(6)-\mathrm{C}(8)-\mathrm{H}(8 \mathrm{~B})$ & 109.5 \\
\hline $\mathrm{C}(6)-\mathrm{P}(2)-\mathrm{C}(10)$ & $108.56(6)$ & $\mathrm{H}(8 \mathrm{~A})-\mathrm{C}(8)-\mathrm{H}(8 \mathrm{~B})$ & 109.5 \\
\hline $\mathrm{O}(2)-\mathrm{P}(2)-\mathrm{Mo}(1)$ & $101.96(3)$ & $\mathrm{C}(6)-\mathrm{C}(8)-\mathrm{H}(8 \mathrm{C})$ & 109.5 \\
\hline $\mathrm{C}(6)-\mathrm{P}(2)-\mathrm{Mo}(1)$ & $125.26(4)$ & $\mathrm{H}(8 \mathrm{~A})-\mathrm{C}(8)-\mathrm{H}(8 \mathrm{C})$ & 109.5 \\
\hline $\mathrm{C}(10)-\mathrm{P}(2)-\mathrm{Mo}(1)$ & $118.76(4)$ & $\mathrm{H}(8 \mathrm{~B})-\mathrm{C}(8)-\mathrm{H}(8 \mathrm{C})$ & 109.5 \\
\hline $\mathrm{C}(1)-\mathrm{O}(1)-\mathrm{P}(1)$ & $120.79(7)$ & $\mathrm{C}(6)-\mathrm{C}(9)-\mathrm{H}(9 \mathrm{~A})$ & 109.5 \\
\hline $\mathrm{C}(5)-\mathrm{O}(2)-\mathrm{P}(2)$ & $120.25(7)$ & $\mathrm{C}(6)-\mathrm{C}(9)-\mathrm{H}(9 \mathrm{~B})$ & 109.5 \\
\hline $\mathrm{C}(5)-\mathrm{N}(1)-\mathrm{C}(1)$ & $117.19(9)$ & $\mathrm{H}(9 \mathrm{~A})-\mathrm{C}(9)-\mathrm{H}(9 \mathrm{~B})$ & 109.5 \\
\hline $\mathrm{C}(5)-\mathrm{N}(1)-\mathrm{Mo}(1)$ & $121.26(7)$ & $\mathrm{C}(6)-\mathrm{C}(9)-\mathrm{H}(9 \mathrm{C})$ & 109.5 \\
\hline $\mathrm{C}(1)-\mathrm{N}(1)-\mathrm{Mo}(1)$ & $121.50(7)$ & $\mathrm{H}(9 \mathrm{~A})-\mathrm{C}(9)-\mathrm{H}(9 \mathrm{C})$ & 109.5 \\
\hline $\mathrm{O}(1)-\mathrm{C}(1)-\mathrm{N}(1)$ & $118.79(9)$ & $\mathrm{H}(9 \mathrm{~B})-\mathrm{C}(9)-\mathrm{H}(9 \mathrm{C})$ & 109.5 \\
\hline $\mathrm{O}(1)-\mathrm{C}(1)-\mathrm{C}(2)$ & $117.88(9)$ & $C(13)-C(10)-C(12)$ & $109.05(11)$ \\
\hline $\mathrm{N}(1)-\mathrm{C}(1)-\mathrm{C}(2)$ & $123.32(10)$ & $\mathrm{C}(13)-\mathrm{C}(10)-\mathrm{C}(11)$ & $105.92(11)$ \\
\hline
\end{tabular}




\begin{tabular}{|c|c|c|c|}
\hline$C(12)-C(10)-C(11)$ & $110.14(11)$ & $\mathrm{C}(14)-\mathrm{C}(16)-\mathrm{H}(16 \mathrm{C})$ & 109.5 \\
\hline $\mathrm{C}(13)-\mathrm{C}(10)-\mathrm{P}(2)$ & $115.80(9)$ & $\mathrm{H}(16 \mathrm{~A})-\mathrm{C}(16)-\mathrm{H}(16 \mathrm{C})$ & 109.5 \\
\hline $\mathrm{C}(12)-\mathrm{C}(10)-\mathrm{P}(2)$ & $109.67(9)$ & $\mathrm{H}(16 \mathrm{~B})-\mathrm{C}(16)-\mathrm{H}(16 \mathrm{C})$ & 109.5 \\
\hline$C(11)-C(10)-P(2)$ & $106.10(8)$ & $\mathrm{C}(14)-\mathrm{C}(17)-\mathrm{H}(17 \mathrm{~A})$ & 109.5 \\
\hline $\mathrm{C}(10)-\mathrm{C}(11)-\mathrm{H}(11 \mathrm{~A})$ & 109.5 & $\mathrm{C}(14)-\mathrm{C}(17)-\mathrm{H}(17 \mathrm{~B})$ & 109.5 \\
\hline $\mathrm{C}(10)-\mathrm{C}(11)-\mathrm{H}(11 \mathrm{~B})$ & 109.5 & $\mathrm{H}(17 \mathrm{~A})-\mathrm{C}(17)-\mathrm{H}(17 \mathrm{~B})$ & 109.5 \\
\hline $\mathrm{H}(11 \mathrm{~A})-\mathrm{C}(11)-\mathrm{H}(11 \mathrm{~B})$ & 109.5 & $\mathrm{C}(14)-\mathrm{C}(17)-\mathrm{H}(17 \mathrm{C})$ & 109.5 \\
\hline $\mathrm{C}(10)-\mathrm{C}(11)-\mathrm{H}(11 \mathrm{C})$ & 109.5 & $\mathrm{H}(17 \mathrm{~A})-\mathrm{C}(17)-\mathrm{H}(17 \mathrm{C})$ & 109.5 \\
\hline $\mathrm{H}(11 \mathrm{~A})-\mathrm{C}(11)-\mathrm{H}(11 \mathrm{C})$ & 109.5 & $\mathrm{H}(17 \mathrm{~B})-\mathrm{C}(17)-\mathrm{H}(17 \mathrm{C})$ & 109.5 \\
\hline $\mathrm{H}(11 \mathrm{~B})-\mathrm{C}(11)-\mathrm{H}(11 \mathrm{C})$ & 109.5 & $\mathrm{C}(21)-\mathrm{C}(18)-\mathrm{C}(20)$ & $108.68(11)$ \\
\hline $\mathrm{C}(10)-\mathrm{C}(12)-\mathrm{H}(12 \mathrm{~A})$ & 109.5 & $\mathrm{C}(21)-\mathrm{C}(18)-\mathrm{C}(19)$ & $106.05(11)$ \\
\hline $\mathrm{C}(10)-\mathrm{C}(12)-\mathrm{H}(12 \mathrm{~B})$ & 109.5 & $\mathrm{C}(20)-\mathrm{C}(18)-\mathrm{C}(19)$ & $110.14(11)$ \\
\hline $\mathrm{H}(12 \mathrm{~A})-\mathrm{C}(12)-\mathrm{H}(12 \mathrm{~B})$ & 109.5 & $\mathrm{C}(21)-\mathrm{C}(18)-\mathrm{P}(1)$ & $116.02(9)$ \\
\hline $\mathrm{C}(10)-\mathrm{C}(12)-\mathrm{H}(12 \mathrm{C})$ & 109.5 & $\mathrm{C}(20)-\mathrm{C}(18)-\mathrm{P}(1)$ & $110.07(9)$ \\
\hline $\mathrm{H}(12 \mathrm{~A})-\mathrm{C}(12)-\mathrm{H}(12 \mathrm{C})$ & 109.5 & $\mathrm{C}(19)-\mathrm{C}(18)-\mathrm{P}(1)$ & $105.71(8)$ \\
\hline $\mathrm{H}(12 \mathrm{~B})-\mathrm{C}(12)-\mathrm{H}(12 \mathrm{C})$ & 109.5 & $\mathrm{C}(18)-\mathrm{C}(19)-\mathrm{H}(19 \mathrm{~A})$ & 109.5 \\
\hline $\mathrm{C}(10)-\mathrm{C}(13)-\mathrm{H}(13 \mathrm{~A})$ & 109.5 & $\mathrm{C}(18)-\mathrm{C}(19)-\mathrm{H}(19 \mathrm{~B})$ & 109.5 \\
\hline $\mathrm{C}(10)-\mathrm{C}(13)-\mathrm{H}(13 \mathrm{~B})$ & 109.5 & $\mathrm{H}(19 \mathrm{~A})-\mathrm{C}(19)-\mathrm{H}(19 \mathrm{~B})$ & 109.5 \\
\hline $\mathrm{H}(13 \mathrm{~A})-\mathrm{C}(13)-\mathrm{H}(13 \mathrm{~B})$ & 109.5 & $\mathrm{C}(18)-\mathrm{C}(19)-\mathrm{H}(19 \mathrm{C})$ & 109.5 \\
\hline $\mathrm{C}(10)-\mathrm{C}(13)-\mathrm{H}(13 \mathrm{C})$ & 109.5 & $\mathrm{H}(19 \mathrm{~A})-\mathrm{C}(19)-\mathrm{H}(19 \mathrm{C})$ & 109.5 \\
\hline $\mathrm{H}(13 \mathrm{~A})-\mathrm{C}(13)-\mathrm{H}(13 \mathrm{C})$ & 109.5 & H(19B)-C(19)-H(19C) & 109.5 \\
\hline $\mathrm{H}(13 \mathrm{~B})-\mathrm{C}(13)-\mathrm{H}(13 \mathrm{C})$ & 109.5 & $\mathrm{C}(18)-\mathrm{C}(20)-\mathrm{H}(20 \mathrm{~A})$ & 109.5 \\
\hline$C(15)-C(14)-C(16)$ & $107.81(11)$ & $\mathrm{C}(18)-\mathrm{C}(20)-\mathrm{H}(20 \mathrm{~B})$ & 109.5 \\
\hline$C(15)-C(14)-C(17)$ & $106.98(11)$ & $\mathrm{H}(20 \mathrm{~A})-\mathrm{C}(20)-\mathrm{H}(20 \mathrm{~B})$ & 109.5 \\
\hline$C(16)-C(14)-C(17)$ & $110.52(11)$ & $\mathrm{C}(18)-\mathrm{C}(20)-\mathrm{H}(20 \mathrm{C})$ & 109.5 \\
\hline $\mathrm{C}(15)-\mathrm{C}(14)-\mathrm{P}(1)$ & $108.57(8)$ & $\mathrm{H}(20 \mathrm{~A})-\mathrm{C}(20)-\mathrm{H}(20 \mathrm{C})$ & 109.5 \\
\hline $\mathrm{C}(16)-\mathrm{C}(14)-\mathrm{P}(1)$ & $108.65(9)$ & $\mathrm{H}(20 \mathrm{~B})-\mathrm{C}(20)-\mathrm{H}(20 \mathrm{C})$ & 109.5 \\
\hline $\mathrm{C}(17)-\mathrm{C}(14)-\mathrm{P}(1)$ & 114.11(9) & $\mathrm{C}(18)-\mathrm{C}(21)-\mathrm{H}(21 \mathrm{~A})$ & 109.5 \\
\hline $\mathrm{C}(14)-\mathrm{C}(15)-\mathrm{H}(15 \mathrm{~A})$ & 109.5 & $\mathrm{C}(18)-\mathrm{C}(21)-\mathrm{H}(21 \mathrm{~B})$ & 109.5 \\
\hline $\mathrm{C}(14)-\mathrm{C}(15)-\mathrm{H}(15 \mathrm{~B})$ & 109.5 & $\mathrm{H}(21 \mathrm{~A})-\mathrm{C}(21)-\mathrm{H}(21 \mathrm{~B})$ & 109.5 \\
\hline $\mathrm{H}(15 \mathrm{~A})-\mathrm{C}(15)-\mathrm{H}(15 \mathrm{~B})$ & 109.5 & $\mathrm{C}(18)-\mathrm{C}(21)-\mathrm{H}(21 \mathrm{C})$ & 109.5 \\
\hline $\mathrm{C}(14)-\mathrm{C}(15)-\mathrm{H}(15 \mathrm{C})$ & 109.5 & $\mathrm{H}(21 \mathrm{~A})-\mathrm{C}(21)-\mathrm{H}(21 \mathrm{C})$ & 109.5 \\
\hline $\mathrm{H}(15 \mathrm{~A})-\mathrm{C}(15)-\mathrm{H}(15 \mathrm{C})$ & 109.5 & $\mathrm{H}(21 \mathrm{~B})-\mathrm{C}(21)-\mathrm{H}(21 \mathrm{C})$ & 109.5 \\
\hline $\mathrm{H}(15 \mathrm{~B})-\mathrm{C}(15)-\mathrm{H}(15 \mathrm{C})$ & 109.5 & $\mathrm{O}(3)-\mathrm{C}(22)-\mathrm{Mo}(1)$ & $173.01(11)$ \\
\hline $\mathrm{C}(14)-\mathrm{C}(16)-\mathrm{H}(16 \mathrm{~A})$ & 109.5 & $\mathrm{O}(4)-\mathrm{C}(23)-\mathrm{Mo}(1)$ & $177.46(11)$ \\
\hline $\mathrm{C}(14)-\mathrm{C}(16)-\mathrm{H}(16 \mathrm{~B})$ & 109.5 & $\mathrm{O}(5)-\mathrm{C}(24)-\mathrm{Mo}(1)$ & $168.83(12)$ \\
\hline $\mathrm{H}(16 \mathrm{~A})-\mathrm{C}(16)-\mathrm{H}(16 \mathrm{~B})$ & 109.5 & & \\
\hline
\end{tabular}


Table S-6. Anisotropic displacement parameters $\left(\AA^{2} \times 10^{3}\right)$ for jonrcr01 (2a). The anisotropic displacement factor exponent takes the form: $-2 \pi^{2}\left[\mathrm{~h}^{2} \mathrm{a}^{* 2} \mathrm{U}_{11}+\ldots+2 \mathrm{hka} \mathrm{a}^{*} \mathrm{U}_{12}\right]$

\begin{tabular}{|c|c|c|c|c|c|c|}
\hline & $\mathrm{U}_{11}$ & $\mathrm{U}_{22}$ & $\mathrm{U}_{33}$ & $\mathrm{U}_{23}$ & $\mathrm{U}_{13}$ & $\mathrm{U}_{12}$ \\
\hline Mo1 & $9(1)$ & $9(1)$ & $12(1)$ & $0(1)$ & $5(1)$ & $0(1)$ \\
\hline P1 & $10(1)$ & $9(1)$ & $16(1)$ & $1(1)$ & $6(1)$ & $0(1)$ \\
\hline $\mathrm{P} 2$ & $10(1)$ & $9(1)$ & $13(1)$ & $0(1)$ & $5(1)$ & $0(1)$ \\
\hline O1 & 11(1) & $9(1)$ & $23(1)$ & $0(1)$ & $9(1)$ & $0(1)$ \\
\hline $\mathrm{O} 2$ & 11(1) & $10(1)$ & $19(1)$ & $1(1)$ & $9(1)$ & $1(1)$ \\
\hline O3 & $32(1)$ & $19(1)$ & $15(1)$ & $-2(1)$ & $9(1)$ & $-1(1)$ \\
\hline $\mathrm{O} 4$ & $12(1)$ & $28(1)$ & $32(1)$ & $-7(1)$ & $10(1)$ & $-1(1)$ \\
\hline O5 & $43(1)$ & $27(1)$ & $24(1)$ & $2(1)$ & $24(1)$ & $3(1)$ \\
\hline N1 & $10(1)$ & $10(1)$ & $13(1)$ & $0(1)$ & $5(1)$ & $0(1)$ \\
\hline $\mathrm{C} 1$ & $10(1)$ & $11(1)$ & $14(1)$ & $0(1)$ & $6(1)$ & $0(1)$ \\
\hline $\mathrm{C} 2$ & $10(1)$ & $12(1)$ & $22(1)$ & $1(1)$ & $7(1)$ & $1(1)$ \\
\hline $\mathrm{C} 3$ & $10(1)$ & $14(1)$ & $23(1)$ & $-1(1)$ & $8(1)$ & $-1(1)$ \\
\hline $\mathrm{C} 4$ & 11(1) & $12(1)$ & $20(1)$ & $0(1)$ & $8(1)$ & $-1(1)$ \\
\hline $\mathrm{C} 5$ & 11(1) & $10(1)$ & $13(1)$ & $0(1)$ & $6(1)$ & $0(1)$ \\
\hline C6 & $15(1)$ & $14(1)$ & $18(1)$ & $4(1)$ & $5(1)$ & $1(1)$ \\
\hline $\mathrm{C} 7$ & $21(1)$ & $28(1)$ & $43(1)$ & $11(1)$ & $14(1)$ & $9(1)$ \\
\hline $\mathrm{C} 8$ & $50(1)$ & $20(1)$ & $18(1)$ & $3(1)$ & $3(1)$ & $-5(1)$ \\
\hline C9 & $24(1)$ & $18(1)$ & $29(1)$ & $6(1)$ & $13(1)$ & $1(1)$ \\
\hline $\mathrm{C} 10$ & $19(1)$ & $16(1)$ & $16(1)$ & $-4(1)$ & $10(1)$ & $-2(1)$ \\
\hline $\mathrm{C} 11$ & $23(1)$ & $23(1)$ & $14(1)$ & $-2(1)$ & $6(1)$ & $-3(1)$ \\
\hline $\mathrm{C} 12$ & $32(1)$ & $23(1)$ & $32(1)$ & $-5(1)$ & $24(1)$ & $1(1)$ \\
\hline $\mathrm{C} 13$ & $29(1)$ & $16(1)$ & $24(1)$ & $-6(1)$ & $11(1)$ & $-6(1)$ \\
\hline C14 & $14(1)$ & $12(1)$ & $20(1)$ & $-2(1)$ & $7(1)$ & $-1(1)$ \\
\hline $\mathrm{C} 15$ & $30(1)$ & $16(1)$ & $20(1)$ & $-3(1)$ & $6(1)$ & $0(1)$ \\
\hline $\mathrm{C} 16$ & $21(1)$ & $23(1)$ & $38(1)$ & $-5(1)$ & $14(1)$ & $-9(1)$ \\
\hline $\mathrm{C} 17$ & $22(1)$ & $16(1)$ & $30(1)$ & $-4(1)$ & $10(1)$ & $4(1)$ \\
\hline $\mathrm{C} 18$ & $19(1)$ & $14(1)$ & $18(1)$ & $4(1)$ & $8(1)$ & $1(1)$ \\
\hline C19 & $21(1)$ & $18(1)$ & $17(1)$ & $2(1)$ & $4(1)$ & $3(1)$ \\
\hline $\mathrm{C} 20$ & $30(1)$ & $22(1)$ & $28(1)$ & $3(1)$ & $20(1)$ & $-2(1)$ \\
\hline $\mathrm{C} 21$ & $30(1)$ & $15(1)$ & $24(1)$ & $6(1)$ & $10(1)$ & $5(1)$ \\
\hline $\mathrm{C} 22$ & $15(1)$ & $12(1)$ & $17(1)$ & $-1(1)$ & $8(1)$ & $-1(1)$ \\
\hline $\mathrm{C} 23$ & $14(1)$ & $14(1)$ & $18(1)$ & $-2(1)$ & $7(1)$ & $0(1)$ \\
\hline C24 & $20(1)$ & $15(1)$ & $17(1)$ & $2(1)$ & $10(1)$ & $2(1)$ \\
\hline
\end{tabular}


Table S-7. Hydrogen coordinates (x 104) and isotropic displacement parameters $\left(\AA^{2} \times 10^{3}\right)$ for jonrcr01 (2a).

\begin{tabular}{|c|c|c|c|c|}
\hline & $\mathrm{x}$ & $\mathrm{y}$ & $\mathrm{z}$ & $\mathrm{U}(\mathrm{eq})$ \\
\hline $\mathrm{H} 2 \mathrm{~A}$ & 12635 & 7293 & 2684 & 18 \\
\hline $\mathrm{H} 3 \mathrm{~A}$ & 13487 & 8824 & 2765 & 19 \\
\hline $\mathrm{H} 4 \mathrm{~A}$ & 12595 & 10374 & 2409 & 17 \\
\hline $\mathrm{H} 7 \mathrm{~A}$ & 7757 & 12652 & 516 & 47 \\
\hline H7B & 8473 & 12663 & 1669 & 47 \\
\hline $\mathrm{H} 7 \mathrm{C}$ & 7697 & 11740 & 1170 & 47 \\
\hline $\mathrm{H} 8 \mathrm{~A}$ & 8132 & 11566 & -530 & 52 \\
\hline H8B & 7959 & 10608 & 12 & 52 \\
\hline $\mathrm{H} 8 \mathrm{C}$ & 8979 & 10692 & -91 & 52 \\
\hline H9A & 9368 & 12843 & 324 & 35 \\
\hline H9B & 10262 & 12021 & 817 & 35 \\
\hline H9C & 10047 & 12813 & 1481 & 35 \\
\hline H11A & 11092 & 10979 & 4599 & 32 \\
\hline H11B & 11511 & 10746 & 3842 & 32 \\
\hline $\mathrm{H} 11 \mathrm{C}$ & 10674 & 10015 & 3891 & 32 \\
\hline $\mathrm{H} 12 \mathrm{~A}$ & 9447 & 11630 & 4121 & 38 \\
\hline H12B & 8781 & 10854 & 3263 & 38 \\
\hline $\mathrm{H} 12 \mathrm{C}$ & 8727 & 12051 & 3049 & 38 \\
\hline H13A & 10821 & 12691 & 3905 & 35 \\
\hline H13B & 9998 & 12911 & 2801 & 35 \\
\hline $\mathrm{H} 13 \mathrm{C}$ & 11061 & 12384 & 3038 & 35 \\
\hline $\mathrm{H} 15 \mathrm{~A}$ & 8268 & 5920 & -215 & 36 \\
\hline H15B & 9094 & 6817 & 174 & 36 \\
\hline $\mathrm{H} 15 \mathrm{C}$ & 8027 & 6956 & 179 & 36 \\
\hline H16A & 7771 & 5071 & 885 & 41 \\
\hline H16B & 7705 & 6078 & 1432 & 41 \\
\hline $\mathrm{H} 16 \mathrm{C}$ & 8444 & 5165 & 2040 & 41 \\
\hline H17A & 9445 & 4702 & 826 & 36 \\
\hline H17B & 10064 & 4855 & 1979 & 36 \\
\hline $\mathrm{H} 17 \mathrm{C}$ & 10347 & 5521 & 1278 & 36 \\
\hline
\end{tabular}




$\begin{array}{lrlll}\text { H19A } & 11037 & 7137 & 4828 & 31 \\ \text { H19B } & 10629 & 7998 & 4004 & 31 \\ \text { H19C } & 11483 & 7212 & 4074 & 31 \\ \text { H20A } & 9397 & 6464 & 4393 & 36 \\ \text { H20B } & 8679 & 5985 & 3346 & 36 \\ \text { H20C } & 8765 & 7196 & 3485 & 36 \\ \text { H21A } & 10809 & 5342 & 4354 & 36 \\ \text { H21B } & 11059 & 5478 & 3467 & 36 \\ \text { H21C } & 9997 & 4988 & 3284 & 36 \\ \end{array}$


Table S-8. Torsion angles $\left[{ }^{\circ}\right]$ for jonrcr01 (2a).

\begin{tabular}{|c|c|c|c|}
\hline C14-P1-O1-C1 & $143.68(9)$ & O2-P2-C6-C7 & $-153.99(10)$ \\
\hline C18-P1-O1-C1 & $-106.19(9)$ & C10-P2-C6-C7 & $-53.85(11)$ \\
\hline Mo1-P1-O1-C1 & $14.88(9)$ & Mo1-P2-C6-C7 & $95.34(10)$ \\
\hline $\mathrm{C} 6-\mathrm{P} 2-\mathrm{O} 2-\mathrm{C} 5$ & $-145.40(9)$ & $\mathrm{O} 2-\mathrm{P} 2-\mathrm{C} 10-\mathrm{C} 13$ & $61.19(10)$ \\
\hline $\mathrm{C} 10-\mathrm{P} 2-\mathrm{O} 2-\mathrm{C} 5$ & $104.70(9)$ & $\mathrm{C} 6-\mathrm{P} 2-\mathrm{C} 10-\mathrm{C} 13$ & $-39.50(11)$ \\
\hline Mo1-P2-O2-C5 & $-16.74(9)$ & Mo1-P2-C10-C13 & $168.99(8)$ \\
\hline $\mathrm{P} 1-\mathrm{O} 1-\mathrm{C} 1-\mathrm{N} 1$ & $-13.57(14)$ & $\mathrm{O} 2-\mathrm{P} 2-\mathrm{C} 10-\mathrm{C} 12$ & $-174.91(9)$ \\
\hline $\mathrm{P} 1-\mathrm{O} 1-\mathrm{C} 1-\mathrm{C} 2$ & $167.06(9)$ & $\mathrm{C} 6-\mathrm{P} 2-\mathrm{C} 10-\mathrm{C} 12$ & $84.40(10)$ \\
\hline C5-N1-C1-O1 & $-174.02(10)$ & Mo1-P2-C10-C12 & $-67.11(10)$ \\
\hline Mo1-N1-C1-O1 & $3.56(14)$ & O2-P2-C10-C11 & $-55.98(9)$ \\
\hline C5-N1-C1-C2 & $5.31(16)$ & C6-P2-C10-C11 & $-156.67(8)$ \\
\hline Mo1-N1-C1-C2 & $-177.11(9)$ & Mo1-P2-C10-C11 & $51.82(9)$ \\
\hline $\mathrm{O} 1-\mathrm{C} 1-\mathrm{C} 2-\mathrm{C} 3$ & $176.39(11)$ & O1-P1-C14-C15 & $-86.69(9)$ \\
\hline $\mathrm{N} 1-\mathrm{C} 1-\mathrm{C} 2-\mathrm{C} 3$ & $-2.94(18)$ & C18-P1-C14-C15 & $173.09(9)$ \\
\hline $\mathrm{C} 1-\mathrm{C} 2-\mathrm{C} 3-\mathrm{C} 4$ & $-0.92(18)$ & Mo1-P1-C14-C15 & $23.56(11)$ \\
\hline $\mathrm{C} 2-\mathrm{C} 3-\mathrm{C} 4-\mathrm{C} 5$ & $2.10(18)$ & O1-P1-C14-C16 & $156.31(9)$ \\
\hline $\mathrm{P} 2-\mathrm{O} 2-\mathrm{C} 5-\mathrm{N} 1$ & $14.12(13)$ & C18-P1-C14-C16 & $56.09(10)$ \\
\hline $\mathrm{P} 2-\mathrm{O} 2-\mathrm{C} 5-\mathrm{C} 4$ & $-166.56(8)$ & Mo1-P1-C14-C16 & $-93.44(10)$ \\
\hline $\mathrm{C} 1-\mathrm{N} 1-\mathrm{C} 5-\mathrm{O} 2$ & $175.28(9)$ & O1-P1-C14-C17 & $32.51(10)$ \\
\hline Mo1-N1-C5-O2 & $-2.30(13)$ & C18-P1-C14-C17 & $-67.72(11)$ \\
\hline C1-N1-C5-C4 & $-4.00(16)$ & Mo1-P1-C14-C17 & $142.76(8)$ \\
\hline Mo1-N1-C5-C4 & $178.42(8)$ & O1-P1-C18-C21 & $-61.45(10)$ \\
\hline $\mathrm{C} 3-\mathrm{C} 4-\mathrm{C} 5-\mathrm{O} 2$ & $-178.89(10)$ & C14-P1-C18-C21 & $39.27(11)$ \\
\hline C3-C4-C5-N1 & $0.41(17)$ & Mo1-P1-C18-C21 & $-168.74(8)$ \\
\hline O2-P2-C6-C8 & $88.75(11)$ & O1-P1-C18-C20 & $174.65(9)$ \\
\hline C10-P2-C6-C8 & $-171.12(11)$ & C14-P1-C18-C20 & $-84.63(10)$ \\
\hline Mo1-P2-C6-C8 & $-21.92(13)$ & Mo1-P1-C18-C20 & $67.36(10)$ \\
\hline O2-P2-C6-C9 & $-30.82(10)$ & O1-P1-C18-C19 & $55.74(9)$ \\
\hline C10-P2-C6-C9 & $69.32(11)$ & C14-P1-C18-C19 & $156.45(8)$ \\
\hline Mo1-P2-C6-C9 & $-141.49(8)$ & Mo1-P1-C18-C19 & $-51.55(9)$ \\
\hline
\end{tabular}


REFERENCE NUMBER: jonlm13 (2b)

CRYSTAL STRUCTURE REPORT

$\mathrm{C}_{20} \mathrm{H}_{31}$ Mo N O $\mathrm{P}_{2}$

or

(iPrPONOP)Mo(CO $)_{3}$

Report prepared for:

L. Munjanja, Prof. W. Jones

November 10, 2014

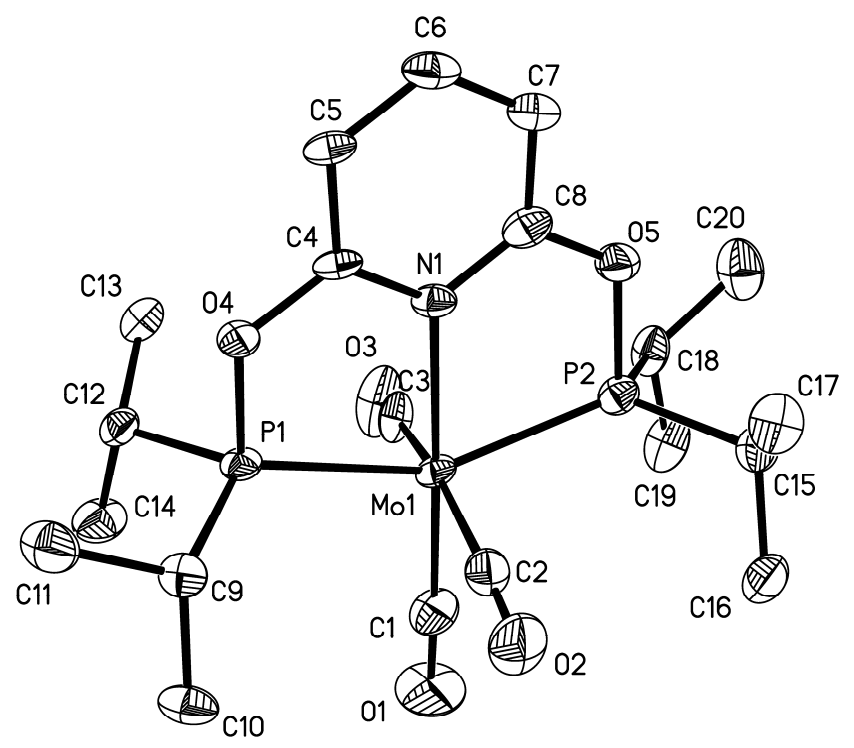

William W. Brennessel

X-ray Crystallographic Facility

Department of Chemistry, University of Rochester

120 Trustee Road

Rochester, NY 14627 


\section{Data collection}

A crystal $\left(0.18 \times 0.14 \times 0.05 \mathrm{~mm}^{3}\right)$ was placed onto the tip of a thin glass optical fiber and mounted on a Bruker SMART APEX II CCD platform diffractometer for a data collection at 100.0(5) K. ${ }^{1}$ A preliminary set of cell constants and an orientation matrix were calculated from reflections harvested from three orthogonal wedges of reciprocal space. The full data collection was carried out using MoK $\alpha$ radiation (graphite monochromator) with a frame time of 60 seconds and a detector distance of $4.02 \mathrm{~cm}$. A randomly oriented region of reciprocal space was surveyed: five major sections of frames were collected with $0.50^{\circ}$ steps in $\omega$ at five different $\phi$ settings and a detector position of $-38^{\circ}$ in $2 \theta$. The intensity data were corrected for absorption. ${ }^{2}$ Final cell constants were calculated from the xyz centroids of 4048 strong reflections from the actual data collection after integration. ${ }^{3}$ See Table S7 for additional crystal and refinement information.

\section{Structure solution and refinement}

The structure was solved using SIR $2011^{4}$ and refined using SHELXL-2014/7.5 The space group $P 2_{1} / n$ was determined based on systematic absences. A direct-methods solution was calculated which provided most nonhydrogen atoms from the E-map. Full-matrix least squares / difference Fourier cycles were performed which located the remaining non-hydrogen atoms. All non-hydrogen atoms were refined with anisotropic displacement parameters. All hydrogen atoms were placed in ideal positions and refined as riding atoms with relative isotropic displacement parameters. The final full matrix least squares refinement converged to $R 1=0.0578\left(F^{2}, I>2 \sigma(I)\right)$ and $w R 2=0.1259\left(F^{2}\right.$, all data $)$.

\section{Structure description}

The structure is the one suggested. The asymmetric unit contains one molecule in a general position.

Unless noted otherwise all structural diagrams containing thermal displacement ellipsoids are drawn at the $50 \%$ probability level.

Data collection, structure solution, and structure refinement were conducted at the X-ray Crystallographic Facility, B51 Hutchison Hall, Department of Chemistry, University of Rochester. All publications arising from this report MUST either 1) include William W. Brennessel as a coauthor or 2) acknowledge William W. Brennessel and the Xray Crystallographic Facility of the Department of Chemistry at the University of Rochester. 
1 APEX2, version 2014.7-1; Bruker AXS: Madison, WI, 2014.

2 Sheldrick, G. M. SADABS, version 2014/3; University of Göttingen: Göttingen, Germany, 2014.

3 SAINT, version 8.32B; Bruker AXS: Madison, WI, 2013.

4 Burla, M. C.; Caliandro, R.; Camalli, M.; Carrozzini, B.; Cascarano, G. L.; Giacovazzo, C.; Mallamo, M.; Mazzone, A.; Polidori, G.; Spagna, R. SIR2011: a new package for crystal structure determination and refinement, version 1.0; Istituto di Cristallografia: Bari, Italy, 2012.

5 Sheldrick, G. M. SHELXL-2014/7; University of Göttingen: Göttingen, Germany, 2014.

Some equations of interest:

$$
\begin{gathered}
R_{\mathrm{int}}=\Sigma\left|F_{\mathrm{o}}^{2}-<F_{\mathrm{o}}^{2}>\right| / \Sigma\left|F_{\mathrm{o}}{ }^{2}\right| \\
R 1=\Sigma|| F_{\mathrm{o}}|-| F_{\mathrm{c}} \| / \Sigma\left|F_{\mathrm{o}}\right| \\
w R 2=\left[\Sigma\left[w\left(F_{\mathrm{o}}{ }^{2}-F_{\mathrm{c}}{ }^{2}\right)^{2}\right] / \Sigma\left[w\left(F_{\mathrm{o}}{ }^{2}\right)^{2}\right]\right]^{1 / 2} \\
\text { where } w=1 /\left[\sigma^{2}\left(F_{\mathrm{o}}{ }^{2}\right)+(a P)^{2}+b P\right] \text { and } \\
P=1 / 3 \max \left(0, F_{\mathrm{o}}{ }^{2}\right)+2 / 3 F_{\mathrm{c}}{ }^{2} \\
\mathrm{GOF}=S=\left[\Sigma\left[w\left(F_{\mathrm{o}}{ }^{2}-F_{\mathrm{c}}{ }^{2}\right)^{2}\right] /(m-n)\right]^{1 / 2}
\end{gathered}
$$

where $m=$ number of reflections and $n=$ number of parameters

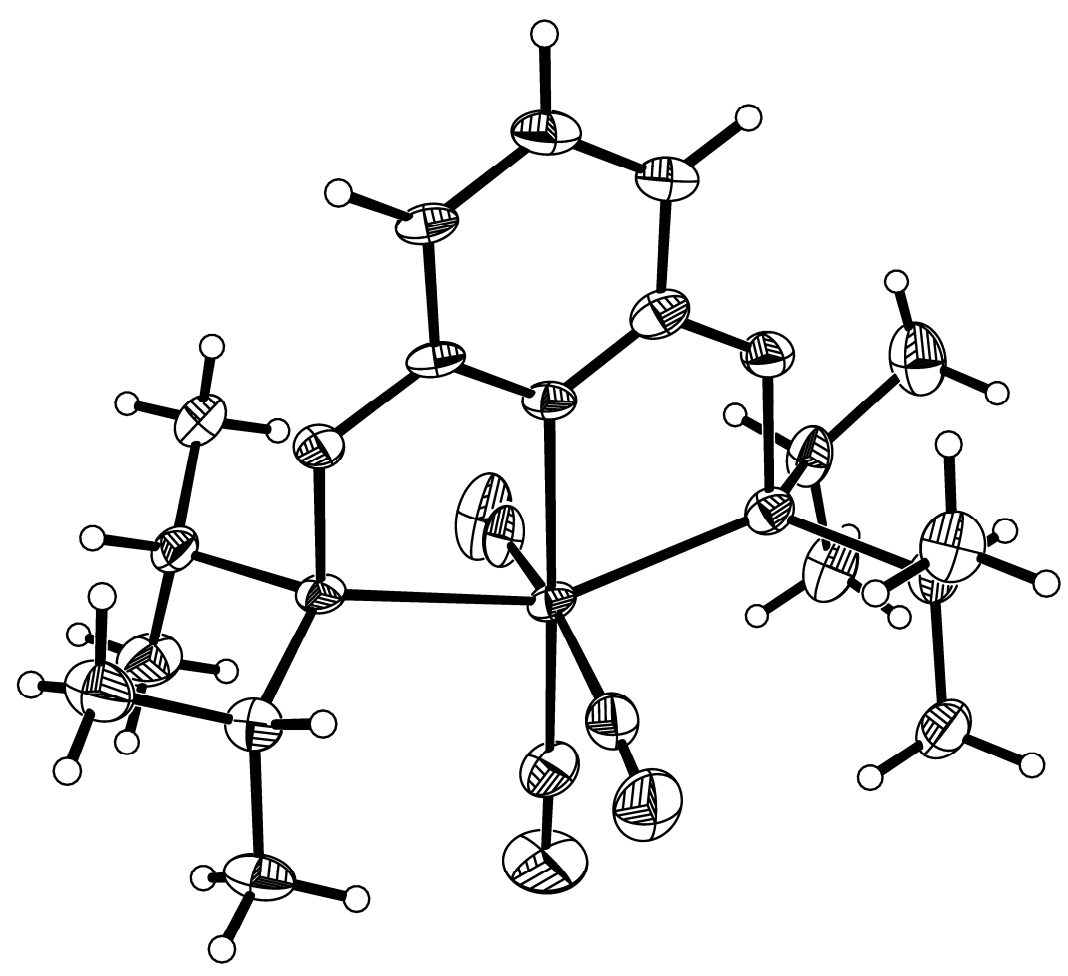


Table S-9. Crystal data and structure refinement for jonlm13. (2b)

\begin{tabular}{|c|c|c|}
\hline Identification code & \multicolumn{2}{|l|}{ jonlm 13} \\
\hline Empirical formula & \multicolumn{2}{|c|}{ C20 H31 Mo N O5 P2 } \\
\hline Formula weight & \multicolumn{2}{|l|}{523.34} \\
\hline Temperature & \multicolumn{2}{|l|}{$100.0(5) \mathrm{K}$} \\
\hline Wavelength & \multicolumn{2}{|l|}{$0.71073 \AA$} \\
\hline Crystal system & \multicolumn{2}{|l|}{ monoclinic } \\
\hline Space group & \multicolumn{2}{|l|}{$P 2_{1} / n$} \\
\hline \multirow[t]{3}{*}{ Unit cell dimensions } & $a=12.482(3) \AA$ & $\alpha=90^{\circ}$ \\
\hline & $b=10.474(3) \AA$ & $\beta=108.701(4)^{\circ}$ \\
\hline & $c=19.450(5) \AA$ & $\gamma=90^{\circ}$ \\
\hline Volume & \multicolumn{2}{|l|}{$2408.7(10) \AA^{3}$} \\
\hline$Z$ & \multicolumn{2}{|l|}{4} \\
\hline Density (calculated) & \multicolumn{2}{|l|}{$1.443 \mathrm{Mg} / \mathrm{m}^{3}$} \\
\hline Absorption coefficient & \multicolumn{2}{|l|}{$0.706 \mathrm{~mm}^{-1}$} \\
\hline$F(000)$ & \multicolumn{2}{|l|}{1080} \\
\hline Crystal color, morphology & \multicolumn{2}{|l|}{ yellow, plate } \\
\hline Crystal size & \multicolumn{2}{|c|}{$0.18 \times 0.14 \times 0.05 \mathrm{~mm}^{3}$} \\
\hline Theta range for data collection & \multicolumn{2}{|l|}{2.211 to $27.581^{\circ}$} \\
\hline Index ranges & \multicolumn{2}{|c|}{$-16 \leq h \leq 16,-13 \leq k \leq 13,-25 \leq l \leq 25$} \\
\hline Reflections collected & \multicolumn{2}{|l|}{38510} \\
\hline Independent reflections & \multicolumn{2}{|c|}{$5567[R(\mathrm{int})=0.1515]$} \\
\hline Observed reflections & \multicolumn{2}{|l|}{3838} \\
\hline Completeness to theta $=27.485^{\circ}$ & \multicolumn{2}{|l|}{$100.0 \%$} \\
\hline Absorption correction & \multicolumn{2}{|l|}{ Multi-scan } \\
\hline Max. and min. transmission & \multicolumn{2}{|l|}{0.7456 and 0.5434} \\
\hline Refinement method & \multicolumn{2}{|c|}{ Full-matrix least-squares on $F^{2}$} \\
\hline Data / restraints / parameters & \multicolumn{2}{|l|}{$5567 / 0 / 262$} \\
\hline Goodness-of-fit on $F^{2}$ & \multicolumn{2}{|l|}{1.076} \\
\hline Final $R$ indices $[I>2 \operatorname{sigma}(I)]$ & \multicolumn{2}{|c|}{$R 1=0.0580, w R 2=0.1127$} \\
\hline$R$ indices (all data) & \multicolumn{2}{|c|}{$R 1=0.0980, w R 2=0.1263$} \\
\hline Largest diff. peak and hole & \multicolumn{2}{|c|}{1.098 and -1.198 e. $\AA^{-3}$} \\
\hline
\end{tabular}


Table S-10. Atomic coordinates (x $\left.10^{4}\right)$ and equivalent isotropic displacement parameters $\left(\AA^{2} \mathrm{x} 10^{3}\right)$ for jonlm13 (2b). $U_{\text {eq }}$ is defined as one third of the trace of the orthogonalized $U_{i j}$ tensor.

\begin{tabular}{|c|c|c|c|c|}
\hline & $\mathrm{x}$ & $\mathrm{y}$ & $\mathrm{z}$ & $\mathrm{U}_{\mathrm{eq}}$ \\
\hline Mo1 & $4160(1)$ & 2351(1) & $1305(1)$ & $19(1)$ \\
\hline $\mathrm{P} 1$ & 5904(1) & $3470(1)$ & $1488(1)$ & $20(1)$ \\
\hline $\mathrm{P} 2$ & 2174(1) & $2262(1)$ & 1121(1) & $23(1)$ \\
\hline $\mathrm{O} 1$ & 4953(4) & $-488(3)$ & 1399(3) & $67(2)$ \\
\hline $\mathrm{O} 2$ & 3938(3) & 2052(4) & $-361(2)$ & $45(1)$ \\
\hline $\mathrm{O} 3$ & $4528(4)$ & 1651(4) & 2942(2) & $53(1)$ \\
\hline $\mathrm{O} 4$ & $5486(3)$ & $5021(3)$ & 1343(2) & $23(1)$ \\
\hline O5 & 1816(3) & $3822(3)$ & 1143(2) & $29(1)$ \\
\hline N1 & $3651(3)$ & $4410(3)$ & $1240(2)$ & $21(1)$ \\
\hline $\mathrm{C} 1$ & $4645(5)$ & $563(5)$ & $1348(3)$ & $37(1)$ \\
\hline $\mathrm{C} 2$ & 3993(4) & $2230(5)$ & $234(3)$ & $29(1)$ \\
\hline $\mathrm{C} 3$ & 4395(4) & $2019(5)$ & 2354(3) & $31(1)$ \\
\hline $\mathrm{C} 4$ & 4399(4) & 5339(4) & $1259(2)$ & $20(1)$ \\
\hline $\mathrm{C} 5$ & 4118(4) & $6637(4)$ & 1212(2) & $24(1)$ \\
\hline $\mathrm{C} 6$ & $3026(4)$ & $6957(5)$ & 1148(3) & $29(1)$ \\
\hline $\mathrm{C} 7$ & $2216(5)$ & 6012(4) & 1120(3) & $28(1)$ \\
\hline $\mathrm{C} 8$ & 2584(4) & $4750(4)$ & 1164(3) & $27(1)$ \\
\hline C9 & 6633(4) & 3394(4) & $805(3)$ & $24(1)$ \\
\hline $\mathrm{C} 10$ & $7019(5)$ & 2032(4) & $708(3)$ & $31(1)$ \\
\hline $\mathrm{C} 11$ & $7592(5)$ & $4367(5)$ & $900(3)$ & $37(1)$ \\
\hline $\mathrm{C} 12$ & 7007(4) & $3683(4)$ & $2369(3)$ & $24(1)$ \\
\hline $\mathrm{C} 13$ & $6524(4)$ & $4223(5)$ & 2936(3) & $30(1)$ \\
\hline $\mathrm{C} 14$ & $7612(5)$ & $2413(5)$ & $2639(3)$ & $38(1)$ \\
\hline $\mathrm{C} 15$ & $1105(4)$ & 1792(5) & $257(3)$ & $27(1)$ \\
\hline $\mathrm{C} 16$ & $1414(5)$ & $489(5)$ & $9(3)$ & $35(1)$ \\
\hline $\mathrm{C} 17$ & $1025(5)$ & $2798(5)$ & $-329(3)$ & $37(1)$ \\
\hline $\mathrm{C} 18$ & 1594(4) & $1704(5)$ & 1824(3) & $31(1)$ \\
\hline C19 & 1671(5) & $250(5)$ & 1905(3) & $40(1)$ \\
\hline $\mathrm{C} 20$ & $406(5)$ & $2190(6)$ & $1755(3)$ & $44(2)$ \\
\hline
\end{tabular}


Table S-11. Bond lengths $[\AA]$ and angles $\left[^{\circ}\right]$ for jonlm13 (2b).

\begin{tabular}{|c|c|c|c|}
\hline $\operatorname{Mo}(1)-C(1)$ & $1.961(5)$ & $\mathrm{C}(11)-\mathrm{H}(11 \mathrm{C})$ & 0.9800 \\
\hline $\operatorname{Mo}(1)-C(3)$ & $1.997(5)$ & $\mathrm{C}(12)-\mathrm{C}(13)$ & $1.525(6)$ \\
\hline $\operatorname{Mo}(1)-C(2)$ & $2.029(5)$ & $C(12)-C(14)$ & $1.536(6)$ \\
\hline $\operatorname{Mo}(1)-\mathrm{N}(1)$ & $2.241(4)$ & $\mathrm{C}(12)-\mathrm{H}(12 \mathrm{~A})$ & 1.0000 \\
\hline Mo(1)-P(2) & $2.3904(14)$ & $\mathrm{C}(13)-\mathrm{H}(13 \mathrm{~A})$ & 0.9800 \\
\hline Mo(1)-P(1) & $2.3957(13)$ & $\mathrm{C}(13)-\mathrm{H}(13 \mathrm{~B})$ & 0.9800 \\
\hline $\mathrm{P}(1)-\mathrm{O}(4)$ & $1.703(3)$ & $\mathrm{C}(13)-\mathrm{H}(13 \mathrm{C})$ & 0.9800 \\
\hline $\mathrm{P}(1)-\mathrm{C}(12)$ & $1.836(5)$ & $\mathrm{C}(14)-\mathrm{H}(14 \mathrm{~A})$ & 0.9800 \\
\hline $\mathrm{P}(1)-\mathrm{C}(9)$ & $1.837(5)$ & $\mathrm{C}(14)-\mathrm{H}(14 \mathrm{~B})$ & 0.9800 \\
\hline $\mathrm{P}(2)-\mathrm{O}(5)$ & $1.698(3)$ & $\mathrm{C}(14)-\mathrm{H}(14 \mathrm{C})$ & 0.9800 \\
\hline $\mathrm{P}(2)-\mathrm{C}(18)$ & $1.837(5)$ & $C(15)-C(17)$ & $1.532(7)$ \\
\hline $\mathrm{P}(2)-\mathrm{C}(15)$ & $1.845(5)$ & $C(15)-C(16)$ & $1.537(7)$ \\
\hline $\mathrm{O}(1)-\mathrm{C}(1)$ & $1.160(6)$ & $\mathrm{C}(15)-\mathrm{H}(15 \mathrm{~A})$ & 1.0000 \\
\hline $\mathrm{O}(2)-\mathrm{C}(2)$ & $1.152(6)$ & $\mathrm{C}(16)-\mathrm{H}(16 \mathrm{~A})$ & 0.9800 \\
\hline $\mathrm{O}(3)-\mathrm{C}(3)$ & $1.167(6)$ & $\mathrm{C}(16)-\mathrm{H}(16 \mathrm{~B})$ & 0.9800 \\
\hline $\mathrm{O}(4)-\mathrm{C}(4)$ & $1.355(5)$ & $\mathrm{C}(16)-\mathrm{H}(16 \mathrm{C})$ & 0.9800 \\
\hline $\mathrm{O}(5)-\mathrm{C}(8)$ & $1.357(6)$ & $\mathrm{C}(17)-\mathrm{H}(17 \mathrm{~A})$ & 0.9800 \\
\hline $\mathrm{N}(1)-\mathrm{C}(8)$ & $1.340(6)$ & $\mathrm{C}(17)-\mathrm{H}(17 \mathrm{~B})$ & 0.9800 \\
\hline $\mathrm{N}(1)-\mathrm{C}(4)$ & $1.341(6)$ & $\mathrm{C}(17)-\mathrm{H}(17 \mathrm{C})$ & 0.9800 \\
\hline $\mathrm{C}(4)-\mathrm{C}(5)$ & $1.400(6)$ & $\mathrm{C}(18)-\mathrm{C}(19)$ & $1.532(7)$ \\
\hline$C(5)-C(6)$ & $1.369(7)$ & $C(18)-C(20)$ & $1.532(7)$ \\
\hline $\mathrm{C}(5)-\mathrm{H}(5 \mathrm{~A})$ & 0.9500 & $\mathrm{C}(18)-\mathrm{H}(18 \mathrm{~A})$ & 1.0000 \\
\hline $\mathrm{C}(6)-\mathrm{C}(7)$ & $1.403(7)$ & $\mathrm{C}(19)-\mathrm{H}(19 \mathrm{~A})$ & 0.9800 \\
\hline $\mathrm{C}(6)-\mathrm{H}(6 \mathrm{~A})$ & 0.9500 & $\mathrm{C}(19)-\mathrm{H}(19 \mathrm{D})$ & 0.9800 \\
\hline $\mathrm{C}(7)-\mathrm{C}(8)$ & $1.393(6)$ & $\mathrm{C}(19)-\mathrm{H}(19 \mathrm{~B})$ & 0.9800 \\
\hline $\mathrm{C}(7)-\mathrm{H}(7 \mathrm{~A})$ & 0.9500 & $\mathrm{C}(20)-\mathrm{H}(20 \mathrm{D})$ & 0.9800 \\
\hline $\mathrm{C}(9)-\mathrm{C}(10)$ & $1.536(6)$ & $\mathrm{C}(20)-\mathrm{H}(20 \mathrm{~A})$ & 0.9800 \\
\hline $\mathrm{C}(9)-\mathrm{C}(11)$ & $1.537(7)$ & $\mathrm{C}(20)-\mathrm{H}(20 \mathrm{~B})$ & 0.9800 \\
\hline $\mathrm{C}(9)-\mathrm{H}(9 \mathrm{~A})$ & 1.0000 & $\mathrm{C}(1)-\mathrm{Mo}(1)-\mathrm{C}(3)$ & $81.2(2)$ \\
\hline $\mathrm{C}(10)-\mathrm{H}(10 \mathrm{~A})$ & 0.9800 & $\mathrm{C}(1)-\mathrm{Mo}(1)-\mathrm{C}(2)$ & $85.0(2)$ \\
\hline $\mathrm{C}(10)-\mathrm{H}(10 \mathrm{~B})$ & 0.9800 & $\mathrm{C}(3)-\mathrm{Mo}(1)-\mathrm{C}(2)$ & $166.1(2)$ \\
\hline $\mathrm{C}(10)-\mathrm{H}(10 \mathrm{C})$ & 0.9800 & $\mathrm{C}(1)-\mathrm{Mo}(1)-\mathrm{N}(1)$ & $178.13(18)$ \\
\hline $\mathrm{C}(11)-\mathrm{H}(11 \mathrm{~A})$ & 0.9800 & $\mathrm{C}(3)-\mathrm{Mo}(1)-\mathrm{N}(1)$ & $99.86(18)$ \\
\hline C(11)-H(11B) & 0.9800 & $\mathrm{C}(2)-\mathrm{Mo}(1)-\mathrm{N}(1)$ & 93.94(17) \\
\hline
\end{tabular}




\begin{tabular}{|c|c|}
\hline $\mathrm{C}(1)-\mathrm{Mo}(1)-\mathrm{P}(2)$ & $105.08(17)$ \\
\hline $\mathrm{C}(3)-\mathrm{Mo}(1)-\mathrm{P}(2)$ & $87.23(15)$ \\
\hline $\mathrm{C}(2)-\mathrm{Mo}(1)-\mathrm{P}(2)$ & $94.77(14)$ \\
\hline $\mathrm{N}(1)-\mathrm{Mo}(1)-\mathrm{P}(2)$ & $76.54(10)$ \\
\hline $\mathrm{C}(1)-\mathrm{Mo}(1)-\mathrm{P}(1)$ & $101.98(17)$ \\
\hline $\mathrm{C}(3)-\mathrm{Mo}(1)-\mathrm{P}(1)$ & $96.03(14)$ \\
\hline $\mathrm{C}(2)-\mathrm{Mo}(1)-\mathrm{P}(1)$ & $88.44(14)$ \\
\hline $\mathrm{N}(1)-\mathrm{Mo}(1)-\mathrm{P}(1)$ & $76.42(10)$ \\
\hline $\mathrm{P}(2)-\mathrm{Mo}(1)-\mathrm{P}(1)$ & $152.93(4)$ \\
\hline $\mathrm{O}(4)-\mathrm{P}(1)-\mathrm{C}(12)$ & $97.87(19)$ \\
\hline $\mathrm{O}(4)-\mathrm{P}(1)-\mathrm{C}(9)$ & $96.77(18)$ \\
\hline $\mathrm{C}(12)-\mathrm{P}(1)-\mathrm{C}(9)$ & $106.5(2)$ \\
\hline $\mathrm{O}(4)-\mathrm{P}(1)-\mathrm{Mo}(1)$ & $103.13(12)$ \\
\hline $\mathrm{C}(12)-\mathrm{P}(1)-\mathrm{Mo}(1)$ & $124.86(16)$ \\
\hline $\mathrm{C}(9)-\mathrm{P}(1)-\mathrm{Mo}(1)$ & $120.58(16)$ \\
\hline $\mathrm{O}(5)-\mathrm{P}(2)-\mathrm{C}(18)$ & $96.7(2)$ \\
\hline $\mathrm{O}(5)-\mathrm{P}(2)-\mathrm{C}(15)$ & $99.3(2)$ \\
\hline $\mathrm{C}(18)-\mathrm{P}(2)-\mathrm{C}(15)$ & $104.5(2)$ \\
\hline $\mathrm{O}(5)-\mathrm{P}(2)-\mathrm{Mo}(1)$ & $103.17(12)$ \\
\hline $\mathrm{C}(18)-\mathrm{P}(2)-\mathrm{Mo}(1)$ & $122.58(18)$ \\
\hline $\mathrm{C}(15)-\mathrm{P}(2)-\mathrm{Mo}(1)$ & $124.00(16)$ \\
\hline $\mathrm{C}(4)-\mathrm{O}(4)-\mathrm{P}(1)$ & $119.7(3)$ \\
\hline $\mathrm{C}(8)-\mathrm{O}(5)-\mathrm{P}(2)$ & $120.0(3)$ \\
\hline $\mathrm{C}(8)-\mathrm{N}(1)-\mathrm{C}(4)$ & $118.0(4)$ \\
\hline $\mathrm{C}(8)-\mathrm{N}(1)-\mathrm{Mo}(1)$ & $121.0(3)$ \\
\hline $\mathrm{C}(4)-\mathrm{N}(1)-\mathrm{Mo}(1)$ & $120.9(3)$ \\
\hline $\mathrm{O}(1)-\mathrm{C}(1)-\mathrm{Mo}(1)$ & $177.6(6)$ \\
\hline $\mathrm{O}(2)-\mathrm{C}(2)-\mathrm{Mo}(1)$ & $173.8(5)$ \\
\hline $\mathrm{O}(3)-\mathrm{C}(3)-\mathrm{Mo}(1)$ & $170.8(5)$ \\
\hline $\mathrm{N}(1)-\mathrm{C}(4)-\mathrm{O}(4)$ & 119.2(4) \\
\hline $\mathrm{N}(1)-\mathrm{C}(4)-\mathrm{C}(5)$ & $123.0(4)$ \\
\hline $\mathrm{O}(4)-\mathrm{C}(4)-\mathrm{C}(5)$ & $117.8(4)$ \\
\hline$C(6)-C(5)-C(4)$ & $117.7(4)$ \\
\hline$C(6)-C(5)-H(5 A)$ & 121.2 \\
\hline $\mathrm{C}(4)-\mathrm{C}(5)-\mathrm{H}(5 \mathrm{~A})$ & 121.2 \\
\hline$C(5)-C(6)-C(7)$ & $121.0(4)$ \\
\hline
\end{tabular}

\begin{tabular}{|c|c|}
\hline $\mathrm{C}(5)-\mathrm{C}(6)-\mathrm{H}(6 \mathrm{~A})$ & 119.5 \\
\hline$C(7)-C(6)-H(6 A)$ & 119.5 \\
\hline$C(8)-C(7)-C(6)$ & $116.6(5)$ \\
\hline $\mathrm{C}(8)-\mathrm{C}(7)-\mathrm{H}(7 \mathrm{~A})$ & 121.7 \\
\hline $\mathrm{C}(6)-\mathrm{C}(7)-\mathrm{H}(7 \mathrm{~A})$ & 121.7 \\
\hline $\mathrm{N}(1)-\mathrm{C}(8)-\mathrm{O}(5)$ & $118.7(4)$ \\
\hline $\mathrm{N}(1)-\mathrm{C}(8)-\mathrm{C}(7)$ & $123.7(5)$ \\
\hline $\mathrm{O}(5)-\mathrm{C}(8)-\mathrm{C}(7)$ & $117.5(5)$ \\
\hline$C(10)-C(9)-C(11)$ & $111.6(4)$ \\
\hline $\mathrm{C}(10)-\mathrm{C}(9)-\mathrm{P}(1)$ & $112.1(3)$ \\
\hline $\mathrm{C}(11)-\mathrm{C}(9)-\mathrm{P}(1)$ & $115.6(3)$ \\
\hline $\mathrm{C}(10)-\mathrm{C}(9)-\mathrm{H}(9 \mathrm{~A})$ & 105.6 \\
\hline $\mathrm{C}(11)-\mathrm{C}(9)-\mathrm{H}(9 \mathrm{~A})$ & 105.6 \\
\hline $\mathrm{P}(1)-\mathrm{C}(9)-\mathrm{H}(9 \mathrm{~A})$ & 105.6 \\
\hline $\mathrm{C}(9)-\mathrm{C}(10)-\mathrm{H}(10 \mathrm{~A})$ & 109.5 \\
\hline $\mathrm{C}(9)-\mathrm{C}(10)-\mathrm{H}(10 \mathrm{~B})$ & 109.5 \\
\hline $\mathrm{H}(10 \mathrm{~A})-\mathrm{C}(10)-\mathrm{H}(10 \mathrm{~B})$ & 109.5 \\
\hline $\mathrm{C}(9)-\mathrm{C}(10)-\mathrm{H}(10 \mathrm{C})$ & 109.5 \\
\hline $\mathrm{H}(10 \mathrm{~A})-\mathrm{C}(10)-\mathrm{H}(10 \mathrm{C})$ & 109.5 \\
\hline $\mathrm{H}(10 \mathrm{~B})-\mathrm{C}(10)-\mathrm{H}(10 \mathrm{C})$ & 109.5 \\
\hline $\mathrm{C}(9)-\mathrm{C}(11)-\mathrm{H}(11 \mathrm{~A})$ & 109.5 \\
\hline C(9)-C(11)-H(11B) & 109.5 \\
\hline $\mathrm{H}(11 \mathrm{~A})-\mathrm{C}(11)-\mathrm{H}(11 \mathrm{~B})$ & 109.5 \\
\hline $\mathrm{C}(9)-\mathrm{C}(11)-\mathrm{H}(11 \mathrm{C})$ & 109.5 \\
\hline $\mathrm{H}(11 \mathrm{~A})-\mathrm{C}(11)-\mathrm{H}(11 \mathrm{C})$ & 109.5 \\
\hline $\mathrm{H}(11 \mathrm{~B})-\mathrm{C}(11)-\mathrm{H}(11 \mathrm{C})$ & 109.5 \\
\hline $\mathrm{C}(13)-\mathrm{C}(12)-\mathrm{C}(14)$ & $109.8(4)$ \\
\hline $\mathrm{C}(13)-\mathrm{C}(12)-\mathrm{P}(1)$ & $111.6(3)$ \\
\hline $\mathrm{C}(14)-\mathrm{C}(12)-\mathrm{P}(1)$ & $110.7(3)$ \\
\hline $\mathrm{C}(13)-\mathrm{C}(12)-\mathrm{H}(12 \mathrm{~A})$ & 108.2 \\
\hline $\mathrm{C}(14)-\mathrm{C}(12)-\mathrm{H}(12 \mathrm{~A})$ & 108.2 \\
\hline $\mathrm{P}(1)-\mathrm{C}(12)-\mathrm{H}(12 \mathrm{~A})$ & 108.2 \\
\hline $\mathrm{C}(12)-\mathrm{C}(13)-\mathrm{H}(13 \mathrm{~A})$ & 109.5 \\
\hline $\mathrm{C}(12)-\mathrm{C}(13)-\mathrm{H}(13 \mathrm{~B})$ & 109.5 \\
\hline $\mathrm{H}(13 \mathrm{~A})-\mathrm{C}(13)-\mathrm{H}(13 \mathrm{~B})$ & 109.5 \\
\hline $\mathrm{C}(12)-\mathrm{C}(13)-\mathrm{H}(13 \mathrm{C})$ & 109.5 \\
\hline
\end{tabular}




$\begin{array}{llll}\mathrm{H}(13 \mathrm{~A})-\mathrm{C}(13)-\mathrm{H}(13 \mathrm{C}) & 109.5 & \mathrm{H}(17 \mathrm{~A})-\mathrm{C}(17)-\mathrm{H}(17 \mathrm{~B}) & 109.5 \\ \mathrm{H}(13 \mathrm{~B})-\mathrm{C}(13)-\mathrm{H}(13 \mathrm{C}) & 109.5 & \mathrm{C}(15)-\mathrm{C}(17)-\mathrm{H}(17 \mathrm{C}) & 109.5 \\ \mathrm{C}(12)-\mathrm{C}(14)-\mathrm{H}(14 \mathrm{~A}) & 109.5 & \mathrm{H}(17 \mathrm{~A})-\mathrm{C}(17)-\mathrm{H}(17 \mathrm{C}) & 109.5 \\ \mathrm{C}(12)-\mathrm{C}(14)-\mathrm{H}(14 \mathrm{~B}) & 109.5 & \mathrm{H}(17 \mathrm{~B})-\mathrm{C}(17)-\mathrm{H}(17 \mathrm{C}) & 109.5 \\ \mathrm{H}(14 \mathrm{~A})-\mathrm{C}(14)-\mathrm{H}(14 \mathrm{~B}) & 109.5 & \mathrm{C}(19)-\mathrm{C}(18)-\mathrm{C}(20) & 111.5(4) \\ \mathrm{C}(12)-\mathrm{C}(14)-\mathrm{H}(14 \mathrm{C}) & 109.5 & \mathrm{C}(19)-\mathrm{C}(18)-\mathrm{P}(2) & 111.5(4) \\ \mathrm{H}(14 \mathrm{~A})-\mathrm{C}(14)-\mathrm{H}(14 \mathrm{C}) & 109.5 & \mathrm{C}(20)-\mathrm{C}(18)-\mathrm{P}(2) & 115.5(4) \\ \mathrm{H}(14 \mathrm{~B})-\mathrm{C}(14)-\mathrm{H}(14 \mathrm{C}) & 109.5 & \mathrm{C}(19)-\mathrm{C}(18)-\mathrm{H}(18 \mathrm{~A}) & 105.8 \\ \mathrm{C}(17)-\mathrm{C}(15)-\mathrm{C}(16) & 109.9(4) & \mathrm{C}(20)-\mathrm{C}(18)-\mathrm{H}(18 \mathrm{~A}) & 105.8 \\ \mathrm{C}(17)-\mathrm{C}(15)-\mathrm{P}(2) & 110.5(3) & \mathrm{P}(2)-\mathrm{C}(18)-\mathrm{H}(18 \mathrm{~A}) & 105.8 \\ \mathrm{C}(16)-\mathrm{C}(15)-\mathrm{P}(2) & 110.0(4) & \mathrm{C}(18)-\mathrm{C}(19)-\mathrm{H}(19 \mathrm{~A}) & 109.5 \\ \mathrm{C}(17)-\mathrm{C}(15)-\mathrm{H}(15 \mathrm{~A}) & 108.8 & \mathrm{C}(18)-\mathrm{C}(19)-\mathrm{H}(19 \mathrm{D}) & 109.5 \\ \mathrm{C}(16)-\mathrm{C}(15)-\mathrm{H}(15 \mathrm{~A}) & 108.8 & \mathrm{H}(19 \mathrm{~A})-\mathrm{C}(19)-\mathrm{H}(19 \mathrm{D}) & 109.5 \\ \mathrm{P}(2)-\mathrm{C}(15)-\mathrm{H}(15 \mathrm{~A}) & 108.8 & \mathrm{C}(18)-\mathrm{C}(19)-\mathrm{H}(19 \mathrm{~B}) & 109.5 \\ \mathrm{C}(15)-\mathrm{C}(16)-\mathrm{H}(16 \mathrm{~A}) & 109.5 & \mathrm{H}(19 \mathrm{~A})-\mathrm{C}(19)-\mathrm{H}(19 \mathrm{~B}) & 109.5 \\ \mathrm{C}(15)-\mathrm{C}(16)-\mathrm{H}(16 \mathrm{~B}) & 109.5 & \mathrm{H}(19 \mathrm{D})-\mathrm{C}(19)-\mathrm{H}(19 \mathrm{~B}) & 109.5 \\ \mathrm{H}(16 \mathrm{~A})-\mathrm{C}(16)-\mathrm{H}(16 \mathrm{~B}) & 109.5 & \mathrm{C}(18)-\mathrm{C}(20)-\mathrm{H}(20 \mathrm{D}) & 109.5 \\ \mathrm{C}(15)-\mathrm{C}(16)-\mathrm{H}(16 \mathrm{C}) & 109.5 & \mathrm{C}(18)-\mathrm{C}(20)-\mathrm{H}(20 \mathrm{~A}) & 109.5 \\ \mathrm{H}(16 \mathrm{~A})-\mathrm{C}(16)-\mathrm{H}(16 \mathrm{C}) & 109.5 & \mathrm{H}(20 \mathrm{D})-\mathrm{C}(20)-\mathrm{H}(20 \mathrm{~A}) & 109.5 \\ \mathrm{H}(16 \mathrm{~B})-\mathrm{C}(16)-\mathrm{H}(16 \mathrm{C}) & 109.5 & \mathrm{C}(18)-\mathrm{C}(20)-\mathrm{H}(20 \mathrm{~B}) & 109.5 \\ \mathrm{C}(15)-\mathrm{C}(17)-\mathrm{H}(17 \mathrm{~A}) & 109.5 & \mathrm{H}(20 \mathrm{D})-\mathrm{C}(20)-\mathrm{H}(20 \mathrm{~B}) & 109.5 \\ \mathrm{C}(15)-\mathrm{C}(17)-\mathrm{H}(17 \mathrm{~B}) & 109.5 & \mathrm{H}(20 \mathrm{~A})-\mathrm{C}(20)-\mathrm{H}(20 \mathrm{~B}) & 109.5\end{array}$

Symmetry transformations used to generate equivalent atoms: 
Table S-12. Anisotropic displacement parameters $\left(\AA^{2} \times 10^{3}\right)$ for jonlm13 (2b). The anisotropic displacement factor exponent takes the form: $-2 \pi^{2}\left[h^{2} a^{* 2} U_{11}+\ldots+2 h k a^{*} b^{*} U_{12}\right]$

\begin{tabular}{|c|c|c|c|c|c|c|}
\hline & $\mathrm{U}_{11}$ & $\mathrm{U}_{22}$ & $\mathrm{U}_{33}$ & $\mathrm{U}_{23}$ & $\mathrm{U}_{13}$ & $\mathrm{U}_{12}$ \\
\hline Mo1 & $26(1)$ & $10(1)$ & $23(1)$ & $-1(1)$ & $10(1)$ & $-2(1)$ \\
\hline P1 & $25(1)$ & $10(1)$ & $26(1)$ & $1(1)$ & $11(1)$ & $0(1)$ \\
\hline P2 & $26(1)$ & $20(1)$ & $24(1)$ & $-1(1)$ & $10(1)$ & $-4(1)$ \\
\hline $\mathrm{O} 1$ & $76(4)$ & 11(2) & $122(4)$ & $0(2)$ & $42(3)$ & $3(2)$ \\
\hline $\mathrm{O} 2$ & $44(2)$ & $66(3)$ & $27(2)$ & $-11(2)$ & $14(2)$ & $-8(2)$ \\
\hline $\mathrm{O} 3$ & $52(3)$ & $72(3)$ & $33(2)$ & $10(2)$ & $10(2)$ & $-27(2)$ \\
\hline $\mathrm{O} 4$ & $23(2)$ & $9(1)$ & $38(2)$ & $2(1)$ & $11(2)$ & $0(1)$ \\
\hline O5 & $26(2)$ & $21(2)$ & $41(2)$ & $-5(2)$ & $14(2)$ & $-1(2)$ \\
\hline N1 & $27(2)$ & $12(2)$ & $25(2)$ & $1(2)$ & $12(2)$ & $2(2)$ \\
\hline $\mathrm{C} 1$ & $42(3)$ & 19(3) & $52(4)$ & $-3(2)$ & $18(3)$ & $-9(2)$ \\
\hline $\mathrm{C} 2$ & $25(3)$ & $27(2)$ & $35(3)$ & $-2(2)$ & $8(2)$ & $-1(2)$ \\
\hline $\mathrm{C} 3$ & $25(3)$ & $34(3)$ & $33(3)$ & $0(2)$ & $6(2)$ & $-12(2)$ \\
\hline $\mathrm{C} 4$ & $32(3)$ & $10(2)$ & $21(2)$ & $-1(2)$ & $12(2)$ & $3(2)$ \\
\hline $\mathrm{C} 5$ & $34(3)$ & $10(2)$ & $28(3)$ & $1(2)$ & $12(2)$ & $2(2)$ \\
\hline C6 & $39(3)$ & $20(2)$ & $29(3)$ & $1(2)$ & $11(2)$ & $10(2)$ \\
\hline $\mathrm{C} 7$ & $34(3)$ & $21(2)$ & $30(3)$ & $-1(2)$ & $13(2)$ & $6(2)$ \\
\hline $\mathrm{C} 8$ & $36(3)$ & $25(2)$ & 21(3) & $-4(2)$ & $12(2)$ & $-3(2)$ \\
\hline C9 & $29(3)$ & $22(2)$ & $25(3)$ & $3(2)$ & $12(2)$ & $1(2)$ \\
\hline C10 & $42(3)$ & $22(2)$ & $37(3)$ & $4(2)$ & $22(3)$ & $9(2)$ \\
\hline C11 & $41(3)$ & $27(3)$ & $52(4)$ & $4(2)$ & $29(3)$ & $-1(2)$ \\
\hline $\mathrm{C} 12$ & $25(3)$ & $17(2)$ & 29(3) & $-3(2)$ & $6(2)$ & $-3(2)$ \\
\hline C13 & $34(3)$ & $26(3)$ & $30(3)$ & $-6(2)$ & $8(2)$ & $-7(2)$ \\
\hline C14 & $44(3)$ & $26(3)$ & $37(3)$ & $1(2)$ & $3(3)$ & $10(3)$ \\
\hline C15 & $26(3)$ & $26(2)$ & $28(3)$ & $2(2)$ & $9(2)$ & $-3(2)$ \\
\hline C16 & $36(3)$ & $35(3)$ & $33(3)$ & $-9(2)$ & $9(3)$ & $-9(2)$ \\
\hline $\mathrm{C} 17$ & $41(3)$ & $40(3)$ & $30(3)$ & $6(2)$ & $10(3)$ & $-3(3)$ \\
\hline $\mathrm{C} 18$ & $28(3)$ & $42(3)$ & $24(3)$ & $-2(2)$ & $10(2)$ & $-12(2)$ \\
\hline C19 & $46(4)$ & $42(3)$ & $31(3)$ & $7(2)$ & $10(3)$ & $-18(3)$ \\
\hline $\mathrm{C} 20$ & $34(3)$ & $65(4)$ & $38(3)$ & $4(3)$ & $17(3)$ & $-6(3)$ \\
\hline
\end{tabular}


Table S-13. Hydrogen coordinates (x 104) and isotropic displacement parameters $\left(\AA^{2} \times 10^{3}\right)$ for jonlm13 (2b).

\begin{tabular}{|c|c|c|c|c|}
\hline & $\mathrm{x}$ & $\mathrm{y}$ & z & $\mathrm{U}(\mathrm{eq})$ \\
\hline H5A & 4665 & 7275 & 1224 & 28 \\
\hline H6A & 2814 & 7831 & 1121 & 35 \\
\hline H7A & 1455 & 6223 & 1073 & 33 \\
\hline H9A & 6048 & 3616 & 333 & 29 \\
\hline $\mathrm{H} 10 \mathrm{~A}$ & 6389 & 1438 & 649 & 47 \\
\hline H10B & 7261 & 1999 & 277 & 47 \\
\hline $\mathrm{H} 10 \mathrm{C}$ & 7651 & 1792 & 1137 & 47 \\
\hline $\mathrm{H} 11 \mathrm{~A}$ & 7318 & 5223 & 960 & 55 \\
\hline H11B & 8229 & 4145 & 1330 & 55 \\
\hline $\mathrm{H} 11 \mathrm{C}$ & 7840 & 4352 & 470 & 55 \\
\hline $\mathrm{H} 12 \mathrm{~A}$ & 7577 & 4301 & 2304 & 29 \\
\hline $\mathrm{H} 13 \mathrm{~A}$ & 6139 & 5032 & 2761 & 45 \\
\hline H13B & 5983 & 3615 & 3021 & 45 \\
\hline $\mathrm{H} 13 \mathrm{C}$ & 7140 & 4369 & 3391 & 45 \\
\hline $\mathrm{H} 14 \mathrm{~A}$ & 7920 & 2070 & 2273 & 57 \\
\hline H14B & 8229 & 2557 & 3093 & 57 \\
\hline $\mathrm{H} 14 \mathrm{C}$ & 7072 & 1803 & 2724 & 57 \\
\hline $\mathrm{H} 15 \mathrm{~A}$ & 353 & 1721 & 335 & 32 \\
\hline H16A & 1464 & -149 & 386 & 52 \\
\hline H16B & 830 & 234 & -441 & 52 \\
\hline $\mathrm{H} 16 \mathrm{C}$ & 2144 & 553 & -76 & 52 \\
\hline H17A & 828 & 3626 & -167 & 55 \\
\hline H17B & 1755 & 2865 & -415 & 55 \\
\hline $\mathrm{H} 17 \mathrm{C}$ & 440 & 2546 & -780 & 55 \\
\hline H18A & 2104 & 2057 & 2293 & 37 \\
\hline H19A & 2439 & -30 & 1948 & 60 \\
\hline H19D & 1492 & -3 & 2342 & 60 \\
\hline H19B & 1130 & -149 & 1478 & 60 \\
\hline H20D & 385 & 3122 & 1704 & 67 \\
\hline $\mathrm{H} 20 \mathrm{~A}$ & -145 & 1807 & 1327 & 67 \\
\hline H20B & 218 & 1953 & 2190 & 67 \\
\hline
\end{tabular}


Table S-14. Torsion angles $\left[{ }^{\circ}\right]$ for jonlm13 (2b).

\begin{tabular}{|c|c|c|c|}
\hline C12-P1-O4-C4 & $-120.5(3)$ & O4-P1-C9-C10 & $-172.9(4)$ \\
\hline C9-P1-O4-C4 & $131.8(3)$ & C12-P1-C9-C10 & $86.8(4)$ \\
\hline Mo1-P1-O4-C4 & $8.1(3)$ & Mo1-P1-C9-C10 & $-63.3(4)$ \\
\hline C18-P2-O5-C8 & $133.5(4)$ & O4-P1-C9-C11 & $57.7(4)$ \\
\hline $\mathrm{C} 15-\mathrm{P} 2-\mathrm{O} 5-\mathrm{C} 8$ & $-120.6(4)$ & C12-P1-C9-C11 & $-42.6(4)$ \\
\hline Mo1-P2-O5-C8 & $7.7(4)$ & Mo1-P1-C9-C11 & $167.3(3)$ \\
\hline C8-N1-C4-O4 & $179.4(4)$ & O4-P1-C12-C13 & $60.3(3)$ \\
\hline Mo1-N1-C4-O4 & $-2.2(6)$ & $\mathrm{C} 9-\mathrm{P} 1-\mathrm{C} 12-\mathrm{C} 13$ & $159.8(3)$ \\
\hline C8-N1-C4-C5 & $0.8(7)$ & Mo1-P1-C12-C13 & $-51.7(4)$ \\
\hline Mo1-N1-C4-C5 & $179.2(3)$ & O4-P1-C12-C14 & $-177.0(3)$ \\
\hline P1-O4-C4-N1 & $-4.7(6)$ & C9-P1-C12-C14 & $-77.6(4)$ \\
\hline P1-O4-C4-C5 & $173.9(3)$ & Mo1-P1-C12-C14 & $70.9(4)$ \\
\hline N1-C4-C5-C6 & $0.3(7)$ & O5-P2-C15-C17 & $45.0(4)$ \\
\hline O4-C4-C5-C6 & $-178.2(4)$ & C18-P2-C15-C17 & $144.5(4)$ \\
\hline C4-C5-C6-C7 & $-0.9(7)$ & Mo1-P2-C15-C17 & $-67.8(4)$ \\
\hline C5-C6-C7-C8 & $0.3(7)$ & O5-P2-C15-C16 & $166.5(3)$ \\
\hline C4-N1-C8-O5 & $180.0(4)$ & C18-P2-C15-C16 & $-94.1(4)$ \\
\hline Mo1-N1-C8-O5 & $1.6(6)$ & Mo1-P2-C15-C16 & $53.7(4)$ \\
\hline C4-N1-C8-C7 & $-1.5(7)$ & O5-P2-C18-C19 & $174.6(4)$ \\
\hline Mo1-N1-C8-C7 & $-179.9(4)$ & C15-P2-C18-C19 & $73.2(4)$ \\
\hline P2-O5-C8-N1 & $-6.7(6)$ & Mo1-P2-C18-C19 & $-75.1(4)$ \\
\hline P2-O5-C8-C7 & $174.7(3)$ & O5-P2-C18-C20 & $46.0(4)$ \\
\hline C6-C7-C8-N1 & $1.0(7)$ & C15-P2-C18-C20 & $-55.4(5)$ \\
\hline C6-C7-C8-O5 & $179.5(4)$ & Mo1-P2-C18-C20 & $156.3(3)$ \\
\hline
\end{tabular}


REFERENCE NUMBER: jonsc14 (2c)

CRYSTAL STRUCTURE REPORT

$$
\mathrm{C}_{24} \mathrm{H}_{39} \mathrm{~N} \mathrm{O}_{5} \mathrm{P}_{2} \mathrm{~W}
$$

or

(tBuPONOP)W(CO)

Report prepared for:

Dr. S. Chakraborty, Prof. W. Jones

February 18, 2014

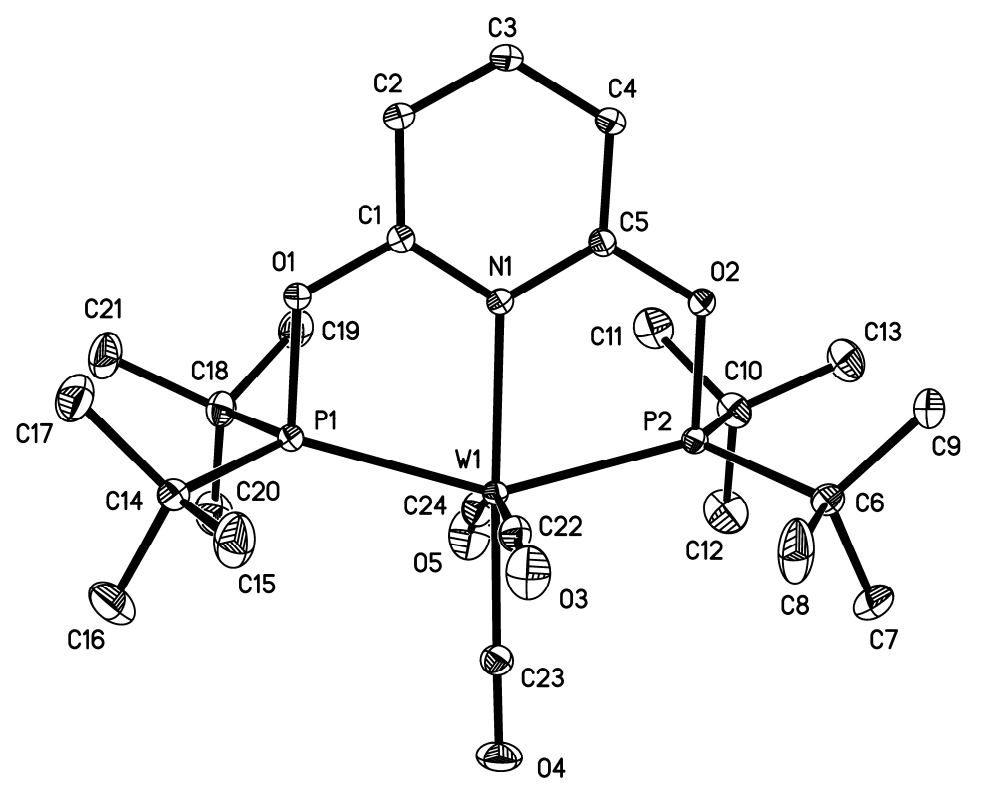

William W. Brennessel

X-ray Crystallographic Facility

Department of Chemistry, University of Rochester

120 Trustee Road

Rochester, NY 14627 


\section{Data collection}

A crystal $\left(0.20 \times 0.20 \times 0.16 \mathrm{~mm}^{3}\right)$ was placed onto the tip of a $0.1 \mathrm{~mm}$ diameter glass capillary tube or fiber and mounted on a Bruker SMART APEX II CCD platform diffractometer for a data collection at 100.0(5) K. ${ }^{1}$ A preliminary set of cell constants and an orientation matrix were calculated from reflections harvested from three orthogonal wedges of reciprocal space. The full data collection was carried out using MoK $\alpha$ radiation (graphite monochromator) with a frame time of 15 seconds and a detector distance of $3.99 \mathrm{~cm}$. A randomly oriented region of reciprocal space was surveyed: six major sections of frames were collected with $0.50^{\circ}$ steps in $\omega$ at six different $\phi$ settings and a detector position of $-38^{\circ}$ in $2 \theta$. The intensity data were corrected for absorption. ${ }^{2}$ Final cell constants were calculated from the xyz centroids of 3889 strong reflections from the actual data collection after integration. ${ }^{3}$ See Table S13 for additional crystal and refinement information.

\section{Structure solution and refinement}

The structure was solved using SIR20114 and refined using SHELXL-2014. ${ }^{5}$ The space group $P 2_{1} / n$ was determined based on systematic absences. A direct-methods solution was calculated which provided most nonhydrogen atoms from the E-map. Full-matrix least squares / difference Fourier cycles were performed which located the remaining non-hydrogen atoms. All non-hydrogen atoms were refined with anisotropic displacement parameters. All hydrogen atoms were placed in ideal positions and refined as riding atoms with relative isotropic displacement parameters.

A lone peak of 5.18 electrons per $\AA^{3}$, located $1.32 \AA$ from atom H8D, has no chemical meaning and is likely due to low angle systematic contamination from a small misaligned region of the crystal (due to cracking upon placement into the coldstream). Because the quality of the overall structure was sufficient for the chemist's needs, a second crystal was not examined. Pseudomerohedral twinning was also considered because the cell can metrically transform to centered orthorhombic by a 180 degree rotation about direct lattice [100]. When refined as such, the minor component of twinning approached zero, and no improvements to the overall model were observed.

The final full matrix least squares refinement converged to $R 1=0.0245\left(F^{2}, I>2 \sigma(I)\right)$ and $w R 2=0.0574\left(F^{2}\right.$, all data).

\section{Structure description}

The structure is the one suggested. One tert-butyl group is modeled as disordered over two positions (83:17). The structure is isotypic with the molybdenum analog (local code JONRCR01).

Unless noted otherwise all structural diagrams containing thermal displacement ellipsoids are drawn at the $50 \%$ probability level. 
Data collection, structure solution, and structure refinement were conducted at the X-ray Crystallographic Facility, B51 Hutchison Hall, Department of Chemistry, University of Rochester. All publications arising from this report MUST either 1) include William W. Brennessel as a coauthor or 2) acknowledge William W. Brennessel and the Xray Crystallographic Facility of the Department of Chemistry at the University of Rochester.

1 APEX2, version 2013.10-0; Bruker AXS: Madison, WI, 2013.

2 Sheldrick, G. M. SADABS, version 2012/1; University of Göttingen: Göttingen, Germany, 2012.

3 SAINT, version 8.34A; Bruker AXS: Madison, WI, 2013.

4 Burla, M. C.; Caliandro, R.; Camalli, M.; Carrozzini, B.; Cascarano, G. L.; Giacovazzo, C.; Mallamo, M.; Mazzone, A.; Polidori, G.; Spagna, R. SIR2011: a new package for crystal structure determination and refinement, version 1.0; Istituto di Cristallografia: Bari, Italy, 2012.

5 Sheldrick, G. M. SHELXL-2014/1; University of Göttingen: Göttingen, Germany, 2014.

Some equations of interest:

$$
\begin{gathered}
R_{\mathrm{int}}=\Sigma\left|F_{\mathrm{o}}^{2}-<F_{\mathrm{o}}^{2}>\right| / \Sigma\left|F_{\mathrm{o}}{ }^{2}\right| \\
R 1=\Sigma|| F_{\mathrm{o}}|-| F_{\mathrm{c}}|| / \Sigma\left|F_{\mathrm{o}}\right| \\
w R 2=\left[\Sigma\left[w\left(F_{\mathrm{o}}{ }^{2} F_{\mathrm{c}}{ }^{2}\right)^{2}\right] / \Sigma\left[w\left(F_{\mathrm{o}}{ }^{2}\right)^{2}\right]\right]^{1 / 2} \\
\text { where } w=1 /\left[\sigma^{2}\left(F_{\mathrm{o}}{ }^{2}\right)+(a P)^{2}+b P\right] \text { and } \\
P=1 / 3 \max \left(0, F_{\mathrm{o}}{ }^{2}\right)+2 / 3 F_{\mathrm{c}}^{2} \\
\mathrm{GOF}=S=\left[\Sigma\left[w\left(F_{\mathrm{o}}{ }^{2}-F_{\mathrm{c}}{ }^{2}\right)^{2}\right] /(m-n)\right]^{1 / 2}
\end{gathered}
$$

where $m=$ number of reflections and $n=$ number of parameters

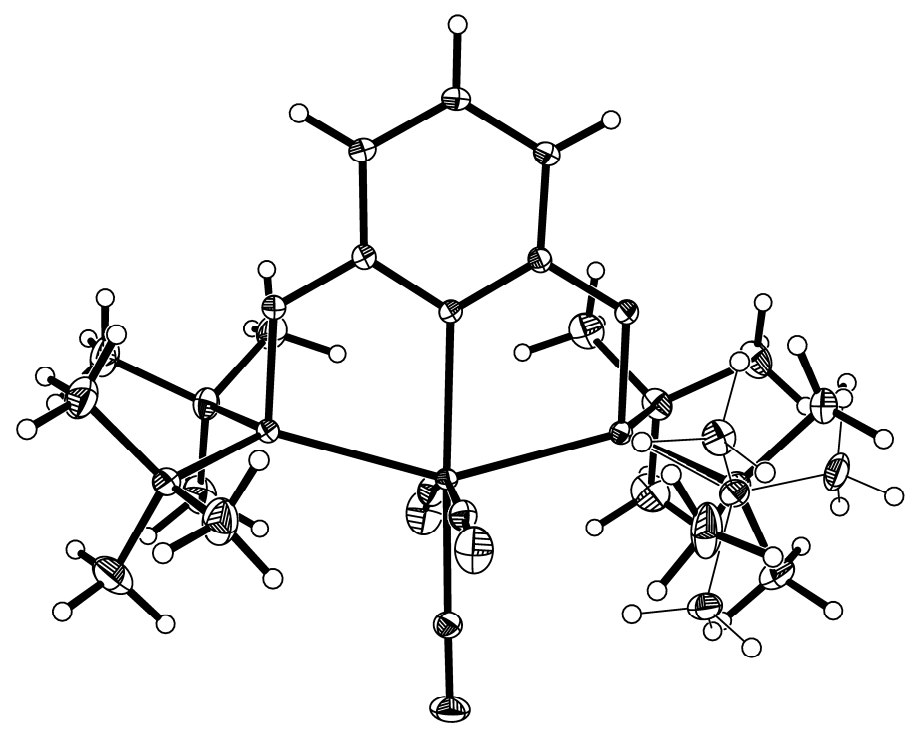


Table S-15. Crystal data and structure refinement for jonsc14.

\begin{tabular}{|c|c|c|}
\hline Identification code & \multicolumn{2}{|l|}{ jonsc 14} \\
\hline Empirical formula & \multicolumn{2}{|c|}{ C24 H39 N O5 P2 W } \\
\hline Formula weight & \multicolumn{2}{|l|}{667.35} \\
\hline Temperature & \multicolumn{2}{|l|}{$100.0(5) \mathrm{K}$} \\
\hline Wavelength & \multicolumn{2}{|l|}{$0.71073 \AA$} \\
\hline Crystal system & \multicolumn{2}{|l|}{ monoclinic } \\
\hline Space group & \multicolumn{2}{|l|}{$P 2_{1} / n$} \\
\hline \multirow[t]{3}{*}{ Unit cell dimensions } & $a=14.8572(16) \AA$ & $\alpha=90^{\circ}$ \\
\hline & $b=13.1404(14) \AA$ & $\beta=117.8272(19)^{\circ}$ \\
\hline & $c=15.5556(16) \AA$ & $\gamma=90^{\circ}$ \\
\hline Volume & \multicolumn{2}{|l|}{$2685.7(5) \AA^{3}$} \\
\hline$Z$ & \multicolumn{2}{|l|}{4} \\
\hline Density (calculated) & \multicolumn{2}{|l|}{$1.650 \mathrm{Mg} / \mathrm{m}^{3}$} \\
\hline Absorption coefficient & \multicolumn{2}{|l|}{$4.454 \mathrm{~mm}^{-1}$} \\
\hline$F(000)$ & \multicolumn{2}{|l|}{1336} \\
\hline Crystal color, morphology & \multicolumn{2}{|c|}{ yellow-orange, wedge } \\
\hline Crystal size & \multicolumn{2}{|c|}{$0.20 \times 0.20 \times 0.16 \mathrm{~mm}^{3}$} \\
\hline Theta range for data collection & \multicolumn{2}{|l|}{1.566 to $38.656^{\circ}$} \\
\hline Index ranges & \multicolumn{2}{|c|}{$-25 \leq h \leq 25,-22 \leq k \leq 23,-27 \leq l \leq 26$} \\
\hline Reflections collected & \multicolumn{2}{|l|}{99917} \\
\hline Independent reflections & \multicolumn{2}{|c|}{$14883[R($ int $)=0.0359]$} \\
\hline Observed reflections & \multicolumn{2}{|l|}{13102} \\
\hline Completeness to theta $=37.785^{\circ}$ & \multicolumn{2}{|l|}{$99.8 \%$} \\
\hline Absorption correction & \multicolumn{2}{|l|}{ Multi-scan } \\
\hline Max. and min. transmission & \multicolumn{2}{|l|}{0.4405 and 0.3231} \\
\hline Refinement method & \multicolumn{2}{|c|}{ Full-matrix least-squares on $F^{2}$} \\
\hline Data / restraints / parameters & \multicolumn{2}{|l|}{14883 / 7 / 344} \\
\hline Goodness-of-fit on $F^{2}$ & \multicolumn{2}{|l|}{1.088} \\
\hline Final $R$ indices $[I>2 \operatorname{sigma}(I)]$ & \multicolumn{2}{|c|}{$R 1=0.0245, w R 2=0.0555$} \\
\hline$R$ indices (all data) & \multicolumn{2}{|c|}{$R 1=0.0303, w R 2=0.0574$} \\
\hline Largest diff. peak and hole & \multicolumn{2}{|c|}{5.176 and -1.080 e. $\AA^{-3}$} \\
\hline
\end{tabular}


Table S-16. Atomic coordinates (x $\left.10^{4}\right)$ and equivalent isotropic displacement parameters $\left(\AA^{2} \mathrm{x} 10^{3}\right)$ for jonsc14 (2c). $U_{e q}$ is defined as one third of the trace of the orthogonalized $U_{i j}$ tensor.

\begin{tabular}{|c|c|c|c|c|}
\hline & $\mathrm{x}$ & $\mathrm{y}$ & $\mathrm{z}$ & $\mathrm{U}_{\mathrm{eq}}$ \\
\hline W1 & $9126(1)$ & $8886(1)$ & 1888(1) & $9(1)$ \\
\hline $\mathrm{P} 1$ & $9612(1)$ & $7085(1)$ & $2190(1)$ & $12(1)$ \\
\hline P2 & $9600(1)$ & $10676(1)$ & 1979(1) & $10(1)$ \\
\hline O1 & $10779(1)$ & 7090(1) & $2240(1)$ & $15(1)$ \\
\hline $\mathrm{O} 2$ & $10754(1)$ & $10615(1)$ & $2005(1)$ & $13(1)$ \\
\hline O3 & $8273(1)$ & $8748(1)$ & $-397(1)$ & $24(1)$ \\
\hline $\mathrm{O} 4$ & 6811(1) & 8997(1) & $1335(1)$ & $25(1)$ \\
\hline O5 & $8966(2)$ & $9046(1)$ & $3834(1)$ & $30(1)$ \\
\hline N1 & $10763(1)$ & $8857(1)$ & 2193(1) & $10(1)$ \\
\hline $\mathrm{C} 1$ & $11281(1)$ & $7972(1)$ & $2334(1)$ & $12(1)$ \\
\hline $\mathrm{C} 2$ & $12300(1)$ & 7924(1) & $2560(1)$ & $15(1)$ \\
\hline $\mathrm{C} 3$ & $12798(1)$ & $8829(1)$ & $2600(1)$ & $16(1)$ \\
\hline $\mathrm{C} 4$ & $12278(1)$ & $9747(1)$ & $2409(1)$ & $14(1)$ \\
\hline $\mathrm{C} 5$ & $11264(1)$ & $9725(1)$ & $2206(1)$ & $11(1)$ \\
\hline C6a & 8984(2) & $11635(2)$ & $966(2)$ & $15(1)$ \\
\hline $\mathrm{C} 7 \mathrm{a}$ & $8140(2)$ & $12202(2)$ & $1087(2)$ & $28(1)$ \\
\hline C8a & $8479(3)$ & $11060(2)$ & $-1(2)$ & $32(1)$ \\
\hline C9a & $9731(2)$ & $12390(2)$ & $893(2)$ & $21(1)$ \\
\hline C6'e & $8941(7)$ & $11469(6)$ & $839(5)$ & $15(1)$ \\
\hline C7'e & $9339(8)$ & $11111(8)$ & $135(7)$ & $18(2)$ \\
\hline C8'e & $9118(8)$ & $12626(7)$ & $968(8)$ & $22(2)$ \\
\hline C9'e & 7790(7) & $11259(8)$ & $404(8)$ & $20(2)$ \\
\hline $\mathrm{C} 10$ & $10037(1)$ & $11372(1)$ & $3170(1)$ & $17(1)$ \\
\hline $\mathrm{C} 11$ & $10895(2)$ & $10716(2)$ & $3951(1)$ & $23(1)$ \\
\hline $\mathrm{C} 12$ & $9146(2)$ & $11473(2)$ & $3404(2)$ & $29(1)$ \\
\hline $\mathrm{C} 13$ & $10508(2)$ & $12430(1)$ & $3230(2)$ & $24(1)$ \\
\hline C14 & $9025(1)$ & 6031(1) & $1275(1)$ & $16(1)$ \\
\hline $\mathrm{C} 15$ & $8570(2)$ & $6473(2)$ & $255(2)$ & $28(1)$ \\
\hline $\mathrm{C} 16$ & $8155(2)$ & $5544(2)$ & $1408(2)$ & $31(1)$ \\
\hline $\mathrm{C} 17$ & $9789(2)$ & $5203(2)$ & $1338(2)$ & $28(1)$ \\
\hline $\mathrm{C} 18$ & $10028(1)$ & $6557(1)$ & $3451(1)$ & $17(1)$ \\
\hline
\end{tabular}




\begin{tabular}{lrrrr}
$\mathrm{C} 19$ & $10863(2)$ & $7287(1)$ & $4154(1)$ & $22(1)$ \\
$\mathrm{C} 20$ & $9127(2)$ & $6541(2)$ & $3674(2)$ & $26(1)$ \\
$\mathrm{C} 21$ & $10513(2)$ & $5491(2)$ & $3648(2)$ & $26(1)$ \\
$\mathrm{C} 22$ & $8647(1)$ & $8793(1)$ & $445(1)$ & $14(1)$ \\
$\mathrm{C} 23$ & $7666(1)$ & $8944(1)$ & $1519(1)$ & $15(1)$ \\
$\mathrm{C} 24$ & $9130(1)$ & $8985(1)$ & $3177(1)$ & $17(1)$ \\
\hline
\end{tabular}


Table S-17. Bond lengths $[\AA]$ and angles $\left[{ }^{\circ}\right]$ for jonsc14 (2c).

\begin{tabular}{|c|c|c|c|}
\hline $\mathrm{W}(1)-\mathrm{C}(23)$ & $1.9696(17)$ & $\mathrm{C} 8 \mathrm{a}-\mathrm{H} 8 \mathrm{Ba}$ & 0.9800 \\
\hline $\mathrm{W}(1)-\mathrm{C}(24)$ & $2.0073(18)$ & $\mathrm{C} 8 \mathrm{a}-\mathrm{H} 8 \mathrm{Ca}$ & 0.9800 \\
\hline $\mathrm{W}(1)-\mathrm{C}(22)$ & $2.0161(17)$ & C9a-H9Aa & 0.9800 \\
\hline $\mathrm{W}(1)-\mathrm{N}(1)$ & $2.2501(13)$ & C9a-H9Ba & 0.9800 \\
\hline $\mathrm{W}(1)-\mathrm{P}(2)$ & $2.4396(5)$ & $\mathrm{C} 9 \mathrm{a}-\mathrm{H} 9 \mathrm{Ca}$ & 0.9800 \\
\hline $\mathrm{W}(1)-\mathrm{P}(1)$ & $2.4551(5)$ & C6'e-C8'e & $1.540(8)$ \\
\hline $\mathrm{P}(1)-\mathrm{O}(1)$ & $1.6991(12)$ & C6'e-C9'e & $1.541(8)$ \\
\hline $\mathrm{P}(1)-\mathrm{C}(14)$ & $1.8835(17)$ & C6'e-C7'e & $1.542(8)$ \\
\hline $\mathrm{P}(1)-\mathrm{C}(18)$ & $1.8919(17)$ & C7'e-H7De & 0.9800 \\
\hline $\mathrm{P}(2)-\mathrm{O}(2)$ & $1.6978(12)$ & C7'e-H7Ee & 0.9800 \\
\hline$P(2)-C 6 a$ & $1.8881(18)$ & C7'e-H7Fe & 0.9800 \\
\hline $\mathrm{P}(2)-\mathrm{C} 6{ }^{\prime} \mathrm{e}$ & $1.889(5)$ & C8'e-H8De & 0.9800 \\
\hline $\mathrm{P}(2)-\mathrm{C}(10)$ & $1.8890(17)$ & C8'e-H8Ee & 0.9800 \\
\hline $\mathrm{O}(1)-\mathrm{C}(1)$ & $1.3480(19)$ & $\mathrm{C} 8$ 'e-H8Fe & 0.9800 \\
\hline $\mathrm{O}(2)-\mathrm{C}(5)$ & $1.3481(18)$ & C9'e-H9De & 0.9800 \\
\hline $\mathrm{O}(3)-\mathrm{C}(22)$ & $1.161(2)$ & C9'e-H9Ee & 0.9800 \\
\hline $\mathrm{O}(4)-\mathrm{C}(23)$ & $1.167(2)$ & C9'e-H9Fe & 0.9800 \\
\hline $\mathrm{O}(5)-\mathrm{C}(24)$ & $1.160(2)$ & $C(10)-C(12)$ & $1.534(3)$ \\
\hline $\mathrm{N}(1)-\mathrm{C}(1)$ & $1.3549(19)$ & $C(10)-C(13)$ & $1.540(3)$ \\
\hline $\mathrm{N}(1)-\mathrm{C}(5)$ & $1.3558(19)$ & $\mathrm{C}(10)-\mathrm{C}(11)$ & $1.548(3)$ \\
\hline$C(1)-C(2)$ & $1.387(2)$ & $\mathrm{C}(11)-\mathrm{H}(11 \mathrm{~A})$ & 0.9800 \\
\hline$C(2)-C(3)$ & $1.387(2)$ & $\mathrm{C}(11)-\mathrm{H}(11 \mathrm{~B})$ & 0.9800 \\
\hline $\mathrm{C}(2)-\mathrm{H}(2)$ & 0.9500 & $\mathrm{C}(11)-\mathrm{H}(11 \mathrm{C})$ & 0.9800 \\
\hline$C(3)-C(4)$ & $1.387(2)$ & $\mathrm{C}(12)-\mathrm{H}(12 \mathrm{~A})$ & 0.9800 \\
\hline $\mathrm{C}(3)-\mathrm{H}(3)$ & 0.9500 & $\mathrm{C}(12)-\mathrm{H}(12 \mathrm{~B})$ & 0.9800 \\
\hline$C(4)-C(5)$ & $1.389(2)$ & $\mathrm{C}(12)-\mathrm{H}(12 \mathrm{C})$ & 0.9800 \\
\hline $\mathrm{C}(4)-\mathrm{H}(4)$ & 0.9500 & $\mathrm{C}(13)-\mathrm{H}(13 \mathrm{~A})$ & 0.9800 \\
\hline C6a-C8a & $1.531(3)$ & $\mathrm{C}(13)-\mathrm{H}(13 \mathrm{~B})$ & 0.9800 \\
\hline С6a-C9a & $1.531(3)$ & $\mathrm{C}(13)-\mathrm{H}(13 \mathrm{C})$ & 0.9800 \\
\hline C6a-C7a & $1.543(3)$ & $C(14)-C(15)$ & $1.521(3)$ \\
\hline C7a-H7Aa & 0.9800 & $C(14)-C(16)$ & $1.539(3)$ \\
\hline C7a-H7Ba & 0.9800 & $C(14)-C(17)$ & $1.543(3)$ \\
\hline $\mathrm{C} 7 \mathrm{a}-\mathrm{H} 7 \mathrm{Ca}$ & 0.9800 & $\mathrm{C}(15)-\mathrm{H}(15 \mathrm{~A})$ & 0.9800 \\
\hline C8a-H8Aa & 0.9800 & $\mathrm{C}(15)-\mathrm{H}(15 \mathrm{~B})$ & 0.9800 \\
\hline
\end{tabular}




\begin{tabular}{|c|c|c|c|}
\hline $\mathrm{C}(15)-\mathrm{H}(15 \mathrm{C})$ & 0.9800 & $\mathrm{C}(14)-\mathrm{P}(1)-\mathrm{C}(18)$ & $108.98(8)$ \\
\hline $\mathrm{C}(16)-\mathrm{H}(16 \mathrm{~A})$ & 0.9800 & $\mathrm{O}(1)-\mathrm{P}(1)-\mathrm{W}(1)$ & $102.00(4)$ \\
\hline $\mathrm{C}(16)-\mathrm{H}(16 \mathrm{~B})$ & 0.9800 & $\mathrm{C}(14)-\mathrm{P}(1)-\mathrm{W}(1)$ & $124.97(6)$ \\
\hline $\mathrm{C}(16)-\mathrm{H}(16 \mathrm{C})$ & 0.9800 & $\mathrm{C}(18)-\mathrm{P}(1)-\mathrm{W}(1)$ & $118.49(6)$ \\
\hline $\mathrm{C}(17)-\mathrm{H}(17 \mathrm{~A})$ & 0.9800 & $\mathrm{O}(2)-\mathrm{P}(2)-\mathrm{C} 6 \mathrm{a}$ & $98.19(9)$ \\
\hline $\mathrm{C}(17)-\mathrm{H}(17 \mathrm{~B})$ & 0.9800 & $\mathrm{O}(2)-\mathrm{P}(2)-\mathrm{C} 6{ }^{\prime} \mathrm{e}$ & $97.2(3)$ \\
\hline $\mathrm{C}(17)-\mathrm{H}(17 \mathrm{C})$ & 0.9800 & $\mathrm{O}(2)-\mathrm{P}(2)-\mathrm{C}(10)$ & $96.82(7)$ \\
\hline$C(18)-C(20)$ & $1.532(3)$ & C6a-P(2)-C(10) & $107.83(9)$ \\
\hline$C(18)-C(21)$ & $1.539(3)$ & $\mathrm{C}^{\prime} \mathrm{e}-\mathrm{P}(2)-\mathrm{C}(10)$ & $116.3(3)$ \\
\hline$C(18)-C(19)$ & $1.546(3)$ & $\mathrm{O}(2)-\mathrm{P}(2)-\mathrm{W}(1)$ & $102.49(4)$ \\
\hline $\mathrm{C}(19)-\mathrm{H}(19 \mathrm{~A})$ & 0.9800 & C6a-P(2)-W(1) & $125.50(7)$ \\
\hline $\mathrm{C}(19)-\mathrm{H}(19 \mathrm{~B})$ & 0.9800 & C6'e-P(2)-W(1) & $117.9(3)$ \\
\hline$C(19)-H(19 C)$ & 0.9800 & $\mathrm{C}(10)-\mathrm{P}(2)-\mathrm{W}(1)$ & $118.79(6)$ \\
\hline $\mathrm{C}(20)-\mathrm{H}(20 \mathrm{~A})$ & 0.9800 & $\mathrm{C}(1)-\mathrm{O}(1)-\mathrm{P}(1)$ & $120.54(10)$ \\
\hline $\mathrm{C}(20)-\mathrm{H}(20 \mathrm{~B})$ & 0.9800 & $\mathrm{C}(5)-\mathrm{O}(2)-\mathrm{P}(2)$ & $119.92(10)$ \\
\hline $\mathrm{C}(20)-\mathrm{H}(20 \mathrm{C})$ & 0.9800 & $\mathrm{C}(1)-\mathrm{N}(1)-\mathrm{C}(5)$ & $116.98(13)$ \\
\hline $\mathrm{C}(21)-\mathrm{H}(21 \mathrm{~A})$ & 0.9800 & $\mathrm{C}(1)-\mathrm{N}(1)-\mathrm{W}(1)$ & $121.62(10)$ \\
\hline $\mathrm{C}(21)-\mathrm{H}(21 \mathrm{~B})$ & 0.9800 & $\mathrm{C}(5)-\mathrm{N}(1)-\mathrm{W}(1)$ & $121.35(10)$ \\
\hline $\mathrm{C}(21)-\mathrm{H}(21 \mathrm{C})$ & 0.9800 & $\mathrm{O}(1)-\mathrm{C}(1)-\mathrm{N}(1)$ & $118.55(13)$ \\
\hline$C(23)-W(1)-C(24)$ & $77.10(7)$ & $\mathrm{O}(1)-\mathrm{C}(1)-\mathrm{C}(2)$ & $118.08(13)$ \\
\hline$C(23)-W(1)-C(22)$ & $84.82(7)$ & $\mathrm{N}(1)-\mathrm{C}(1)-\mathrm{C}(2)$ & $123.37(14)$ \\
\hline $\mathrm{C}(24)-\mathrm{W}(1)-\mathrm{C}(22)$ & $161.91(7)$ & $C(1)-C(2)-C(3)$ & $117.93(14)$ \\
\hline $\mathrm{C}(23)-\mathrm{W}(1)-\mathrm{N}(1)$ & $175.67(6)$ & $\mathrm{C}(1)-\mathrm{C}(2)-\mathrm{H}(2)$ & 121.0 \\
\hline $\mathrm{C}(24)-\mathrm{W}(1)-\mathrm{N}(1)$ & $106.97(6)$ & $\mathrm{C}(3)-\mathrm{C}(2)-\mathrm{H}(2)$ & 121.0 \\
\hline $\mathrm{C}(22)-\mathrm{W}(1)-\mathrm{N}(1)$ & $91.11(6)$ & $C(4)-C(3)-C(2)$ & $120.32(15)$ \\
\hline $\mathrm{C}(23)-\mathrm{W}(1)-\mathrm{P}(2)$ & $103.22(5)$ & $\mathrm{C}(4)-\mathrm{C}(3)-\mathrm{H}(3)$ & 119.8 \\
\hline $\mathrm{C}(24)-\mathrm{W}(1)-\mathrm{P}(2)$ & $90.76(5)$ & $\mathrm{C}(2)-\mathrm{C}(3)-\mathrm{H}(3)$ & 119.8 \\
\hline $\mathrm{C}(22)-\mathrm{W}(1)-\mathrm{P}(2)$ & $93.79(4)$ & $C(3)-C(4)-C(5)$ & $117.80(14)$ \\
\hline $\mathrm{N}(1)-\mathrm{W}(1)-\mathrm{P}(2)$ & $75.52(3)$ & $\mathrm{C}(3)-\mathrm{C}(4)-\mathrm{H}(4)$ & 121.1 \\
\hline $\mathrm{C}(23)-\mathrm{W}(1)-\mathrm{P}(1)$ & $106.15(5)$ & $\mathrm{C}(5)-\mathrm{C}(4)-\mathrm{H}(4)$ & 121.1 \\
\hline $\mathrm{C}(24)-\mathrm{W}(1)-\mathrm{P}(1)$ & $90.44(5)$ & $\mathrm{O}(2)-\mathrm{C}(5)-\mathrm{N}(1)$ & $118.73(13)$ \\
\hline $\mathrm{C}(22)-\mathrm{W}(1)-\mathrm{P}(1)$ & $94.24(4)$ & $\mathrm{O}(2)-\mathrm{C}(5)-\mathrm{C}(4)$ & $117.86(13)$ \\
\hline $\mathrm{N}(1)-\mathrm{W}(1)-\mathrm{P}(1)$ & $75.58(3)$ & $\mathrm{N}(1)-\mathrm{C}(5)-\mathrm{C}(4)$ & $123.42(13)$ \\
\hline $\mathrm{P}(2)-\mathrm{W}(1)-\mathrm{P}(1)$ & $150.113(14)$ & C8a-C6a-C9a & $106.7(2)$ \\
\hline $\mathrm{O}(1)-\mathrm{P}(1)-\mathrm{C}(14)$ & $98.07(7)$ & C8a-C6a-C7a & $107.7(2)$ \\
\hline $\mathrm{O}(1)-\mathrm{P}(1)-\mathrm{C}(18)$ & $97.02(7)$ & C9a-C6a-C7a & $110.73(18)$ \\
\hline
\end{tabular}




\begin{tabular}{|c|c|}
\hline C8a-C6a-P(2) & $108.37(14)$ \\
\hline С9a-C6а-Р(2) & $114.10(15)$ \\
\hline C7a-C6a-P(2) & $108.99(16)$ \\
\hline C6a-C7a-H7Aa & 109.5 \\
\hline C6a-C7a-H7Ba & 109.5 \\
\hline H7Aa-C7a-H7Ba & 109.5 \\
\hline $\mathrm{C} 6 \mathrm{a}-\mathrm{C} 7 \mathrm{a}-\mathrm{H} 7 \mathrm{Ca}$ & 109.5 \\
\hline $\mathrm{H} 7 \mathrm{Aa}-\mathrm{C} 7 \mathrm{a}-\mathrm{H} 7 \mathrm{Ca}$ & 109.5 \\
\hline H7Ba-C7a-H7Ca & 109.5 \\
\hline С6а-C8a-H8Аa & 109.5 \\
\hline С6a-C8a-H8Ba & 109.5 \\
\hline H8Aa-C8a-H8Ba & 109.5 \\
\hline C6a-C8a-H8Ca & 109.5 \\
\hline H8Aa-C8a-H8Ca & 109.5 \\
\hline H8Ba-C8a-H8Ca & 109.5 \\
\hline С6а-C9a-H9Aa & 109.5 \\
\hline С6а-С9а-H9Ba & 109.5 \\
\hline Н9Aа-С9а-Н9Ba & 109.5 \\
\hline С6а-С9a-H9Ca & 109.5 \\
\hline H9Aa-C9a-H9Ca & 109.5 \\
\hline H9Ba-C9a-H9Ca & 109.5 \\
\hline C8'e-C6'e-C9'e & $108.8(6)$ \\
\hline C8'e-C6'e-C7'e & $107.1(6)$ \\
\hline C9'e-C6'e-C7'e & $110.4(7)$ \\
\hline C8'e-C6'e-P(2) & $116.1(6)$ \\
\hline C9'e-C6'e-P(2) & $107.2(6)$ \\
\hline C7'e-C6'e-P(2) & 107.2(5) \\
\hline C6'e-C7'e-H7De & 109.5 \\
\hline C6'e-C7'e-H7Ee & 109.5 \\
\hline H7De-C7'e-H7Ee & 109.5 \\
\hline C6'e-C7'e-H7Fe & 109.5 \\
\hline H7De-C7'e-H7Fe & 109.5 \\
\hline H7Ee-C7'e-H7Fe & 109.5 \\
\hline C6'e-C8'e-H8De & 109.5 \\
\hline C6'e-C8'e-H8Ee & 109.5 \\
\hline H8De-C8'e-H8Ee & 109.5 \\
\hline
\end{tabular}

\begin{tabular}{ll} 
C6'e-C8'e-H8Fe & 109.5 \\
H8De-C8'e-H8Fe & 109.5 \\
H8Ee-C8'e-H8Fe & 109.5 \\
C6'e-C9'e-H9De & 109.5 \\
C6'e-C9'e-H9Ee & 109.5 \\
H9De-C9'e-H9Ee & 109.5 \\
C6'e-C9'e-H9Fe & 109.5 \\
H9De-C9'e-H9Fe & 109.5 \\
H9Ee-C9'e-H9Fe & 109.5 \\
C(12)-C(10)-C(13) & $109.02(16)$ \\
C(12)-C(10)-C(11) & $110.34(17)$ \\
C(13)-C(10)-C(11) & $105.69(15)$ \\
C(12)-C(10)-P(2) & $109.61(13)$ \\
C(13)-C(10)-P(2) & $115.78(13)$ \\
C(11)-C(10)-P(2) & $106.24(12)$ \\
C(10)-C(11)-H(11A) & 109.5 \\
C(10)-C(11)-H(11B) & 109.5 \\
H(11A)-C(11)-H(11B) & 109.5 \\
C(10)-C(11)-H(11C) & 109.5 \\
H(11A)-C(11)-H(11C) & 109.5 \\
H(11B)-C(11)-H(11C) & 109.5 \\
C(10)-C(12)-H(12A) & 109.5 \\
C(10)-C(12)-H(12B) & 109.5 \\
H(12A)-C(12)-H(12B) & 109.5 \\
C(10)-C(12)-H(12C) & 109.5 \\
H(12A)-C(12)-H(12C) & 109.5 \\
H(12B)-C(12)-H(12C) & 109.5 \\
C(10)-C(13)-H(13A) & 109.5 \\
C(10)-C(13)-H(13B) & 109.5 \\
H(13A)-C(13)-H(13B) & 109.5 \\
C(10)-C(13)-H(13C) & 109.5 \\
H(13A)-C(13)-H(13C) & 109.5 \\
H(14)-C(13)-H(13C) & 109.5 \\
C(17) & $107.89(17)$ \\
\hline
\end{tabular}




$\begin{array}{llll}\mathrm{C}(15)-\mathrm{C}(14)-\mathrm{P}(1) & 109.33(12) & \mathrm{C}(20)-\mathrm{C}(18)-\mathrm{P}(1) & 110.08(13) \\ \mathrm{C}(16)-\mathrm{C}(14)-\mathrm{P}(1) & 109.01(14) & \mathrm{C}(21)-\mathrm{C}(18)-\mathrm{P}(1) & 115.87(13) \\ \mathrm{C}(17)-\mathrm{C}(14)-\mathrm{P}(1) & 113.76(13) & \mathrm{C}(19)-\mathrm{C}(18)-\mathrm{P}(1) & 105.72(12) \\ \mathrm{C}(14)-\mathrm{C}(15)-\mathrm{H}(15 \mathrm{~A}) & 109.5 & \mathrm{C}(18)-\mathrm{C}(19)-\mathrm{H}(19 \mathrm{~A}) & 109.5 \\ \mathrm{C}(14)-\mathrm{C}(15)-\mathrm{H}(15 \mathrm{~B}) & 109.5 & \mathrm{C}(18)-\mathrm{C}(19)-\mathrm{H}(19 \mathrm{~B}) & 109.5 \\ \mathrm{H}(15 \mathrm{~A})-\mathrm{C}(15)-\mathrm{H}(15 \mathrm{~B}) & 109.5 & \mathrm{H}(19 \mathrm{~A})-\mathrm{C}(19)-\mathrm{H}(19 \mathrm{~B}) & 109.5 \\ \mathrm{C}(14)-\mathrm{C}(15)-\mathrm{H}(15 \mathrm{C}) & 109.5 & \mathrm{C}(18)-\mathrm{C}(19)-\mathrm{H}(19 \mathrm{C}) & 109.5 \\ \mathrm{H}(15 \mathrm{~A})-\mathrm{C}(15)-\mathrm{H}(15 \mathrm{C}) & 109.5 & \mathrm{H}(19 \mathrm{~A})-\mathrm{C}(19)-\mathrm{H}(19 \mathrm{C}) & 109.5 \\ \mathrm{H}(15 \mathrm{~B})-\mathrm{C}(15)-\mathrm{H}(15 \mathrm{C}) & 109.5 & \mathrm{H}(19 \mathrm{~B})-\mathrm{C}(19)-\mathrm{H}(19 \mathrm{C}) & 109.5 \\ \mathrm{C}(14)-\mathrm{C}(16)-\mathrm{H}(16 \mathrm{~A}) & 109.5 & \mathrm{C}(18)-\mathrm{C}(20)-\mathrm{H}(20 \mathrm{~A}) & 109.5 \\ \mathrm{C}(14)-\mathrm{C}(16)-\mathrm{H}(16 \mathrm{~B}) & 109.5 & \mathrm{C}(18)-\mathrm{C}(20)-\mathrm{H}(20 \mathrm{~B}) & 109.5 \\ \mathrm{H}(16 \mathrm{~A})-\mathrm{C}(16)-\mathrm{H}(16 \mathrm{~B}) & 109.5 & \mathrm{H}(20 \mathrm{~A})-\mathrm{C}(20)-\mathrm{H}(20 \mathrm{~B}) & 109.5 \\ \mathrm{C}(14)-\mathrm{C}(16)-\mathrm{H}(16 \mathrm{C}) & 109.5 & \mathrm{C}(18)-\mathrm{C}(20)-\mathrm{H}(20 \mathrm{C}) & 109.5 \\ \mathrm{H}(16 \mathrm{~A})-\mathrm{C}(16)-\mathrm{H}(16 \mathrm{C}) & 109.5 & \mathrm{H}(20 \mathrm{~A})-\mathrm{C}(20)-\mathrm{H}(20 \mathrm{C}) & 109.5 \\ \mathrm{H}(16 \mathrm{~B})-\mathrm{C}(16)-\mathrm{H}(16 \mathrm{C}) & 109.5 & \mathrm{H}(20 \mathrm{~B})-\mathrm{C}(20)-\mathrm{H}(20 \mathrm{C}) & 109.5 \\ \mathrm{C}(14)-\mathrm{C}(17)-\mathrm{H}(17 \mathrm{~A}) & 109.5 & \mathrm{C}(18)-\mathrm{C}(21)-\mathrm{H}(21 \mathrm{~A}) & 109.5 \\ \mathrm{C}(14)-\mathrm{C}(17)-\mathrm{H}(17 \mathrm{~B}) & 109.5 & \mathrm{C}(18)-\mathrm{C}(21)-\mathrm{H}(21 \mathrm{~B}) & 109.5 \\ \mathrm{H}(17 \mathrm{~A})-\mathrm{C}(17)-\mathrm{H}(17 \mathrm{~B}) & 109.5 & \mathrm{H}(21 \mathrm{~A})-\mathrm{C}(21)-\mathrm{H}(21 \mathrm{~B}) & 109.5 \\ \mathrm{C}(14)-\mathrm{C}(17)-\mathrm{H}(17 \mathrm{C}) & 109.5 & \mathrm{C}(18)-\mathrm{C}(21)-\mathrm{H}(21 \mathrm{C}) & 109.5 \\ \mathrm{H}(17 \mathrm{~A})-\mathrm{C}(17)-\mathrm{H}(17 \mathrm{C}) & 109.5 & \mathrm{H}(21 \mathrm{~A})-\mathrm{C}(21)-\mathrm{H}(21 \mathrm{C}) & 109.5 \\ \mathrm{H}(17 \mathrm{~B})-\mathrm{C}(17)-\mathrm{H}(17 \mathrm{C}) & 109.5 & \mathrm{H}(21 \mathrm{~B})-\mathrm{C}(21)-\mathrm{H}(21 \mathrm{C}) & 109.5 \\ \mathrm{C}(20)-\mathrm{C}(18)-\mathrm{C}(21) & 108.68(16) & \mathrm{O}(3)-\mathrm{C}(22)-\mathrm{W}(1) & 173.16(16) \\ \mathrm{C}(20)-\mathrm{C}(18)-\mathrm{C}(19) & 110.20(16) & \mathrm{O}(4)-\mathrm{C}(23)-\mathrm{W}(1) & 177.32(15) \\ \mathrm{C}(21)-\mathrm{C}(18)-\mathrm{C}(19) & 106.13(15) & \mathrm{O}(5)-\mathrm{C}(24)-\mathrm{W}(1) & 169.17(17) \\ & & & \\ & & & \end{array}$


Table S-18. Anisotropic displacement parameters $\left(\AA^{2} \times 10^{3}\right)$ for jonsc14 (2c). The anisotropic displacement factor exponent takes the form: $-2 \pi^{2}\left[\mathrm{~h}^{2} \mathrm{a}^{* 2} \mathrm{U}_{11}+\ldots+2 \mathrm{~h} \mathrm{k} \mathrm{a}^{*} \mathrm{~b}^{*} \mathrm{U}_{12}\right]$

\begin{tabular}{|c|c|c|c|c|c|c|}
\hline & $\mathrm{U}_{11}$ & $\mathrm{U}_{22}$ & $\mathrm{U}_{33}$ & $\mathrm{U}_{23}$ & $\mathrm{U}_{13}$ & $\mathrm{U}_{12}$ \\
\hline W1 & $8(1)$ & $9(1)$ & $11(1)$ & $0(1)$ & $5(1)$ & $0(1)$ \\
\hline P1 & $10(1)$ & $10(1)$ & $16(1)$ & 1(1) & $6(1)$ & $0(1)$ \\
\hline $\mathrm{P} 2$ & $10(1)$ & $9(1)$ & $12(1)$ & $-1(1)$ & $5(1)$ & $0(1)$ \\
\hline $\mathrm{O} 1$ & 11(1) & $10(1)$ & $24(1)$ & 1(1) & $9(1)$ & $0(1)$ \\
\hline $\mathrm{O} 2$ & $12(1)$ & $10(1)$ & $20(1)$ & 1(1) & $9(1)$ & $1(1)$ \\
\hline $\mathrm{O} 3$ & $32(1)$ & $22(1)$ & $14(1)$ & $-2(1)$ & $7(1)$ & $-2(1)$ \\
\hline $\mathrm{O} 4$ & $13(1)$ & $28(1)$ & $34(1)$ & $-6(1)$ & $11(1)$ & $-1(1)$ \\
\hline O5 & $46(1)$ & $30(1)$ & $24(1)$ & 1(1) & $25(1)$ & $3(1)$ \\
\hline N1 & $10(1)$ & $10(1)$ & $13(1)$ & $0(1)$ & $6(1)$ & $0(1)$ \\
\hline $\mathrm{C} 1$ & $11(1)$ & $10(1)$ & $15(1)$ & $0(1)$ & $6(1)$ & $0(1)$ \\
\hline $\mathrm{C} 2$ & $10(1)$ & $13(1)$ & $23(1)$ & 1(1) & $7(1)$ & $1(1)$ \\
\hline $\mathrm{C} 3$ & $10(1)$ & $14(1)$ & $25(1)$ & $0(1)$ & $8(1)$ & $0(1)$ \\
\hline $\mathrm{C} 4$ & $11(1)$ & $12(1)$ & $20(1)$ & $-1(1)$ & $8(1)$ & $-1(1)$ \\
\hline $\mathrm{C} 5$ & $12(1)$ & $10(1)$ & $13(1)$ & $0(1)$ & $6(1)$ & $-1(1)$ \\
\hline C6a & $14(1)$ & $13(1)$ & $16(1)$ & $2(1)$ & $5(1)$ & $0(1)$ \\
\hline $\mathrm{C} 7 \mathrm{a}$ & $17(1)$ & $25(1)$ & $40(1)$ & $10(1)$ & $13(1)$ & $8(1)$ \\
\hline $\mathrm{C} 8 \mathrm{a}$ & $48(2)$ & $18(1)$ & $15(1)$ & $3(1)$ & $2(1)$ & $-4(1)$ \\
\hline C9a & $21(1)$ & $16(1)$ & $25(1)$ & $6(1)$ & $10(1)$ & $1(1)$ \\
\hline C6'e & $14(1)$ & $13(1)$ & $16(1)$ & $2(1)$ & $5(1)$ & $0(1)$ \\
\hline C7'e & $21(4)$ & $24(4)$ & $13(4)$ & $7(3)$ & $12(3)$ & $2(4)$ \\
\hline C8'e & $24(5)$ & $11(4)$ & $25(5)$ & $4(3)$ & $7(4)$ & $4(3)$ \\
\hline C9'e & $13(4)$ & $24(5)$ & $24(5)$ & $5(4)$ & $8(4)$ & $2(3)$ \\
\hline $\mathrm{C} 10$ & $20(1)$ & $16(1)$ & $17(1)$ & $-6(1)$ & $11(1)$ & $-3(1)$ \\
\hline $\mathrm{C} 11$ & $25(1)$ & $26(1)$ & $13(1)$ & $-3(1)$ & $5(1)$ & $-4(1)$ \\
\hline $\mathrm{C} 12$ & $34(1)$ & $28(1)$ & $37(1)$ & $-10(1)$ & $28(1)$ & $-2(1)$ \\
\hline $\mathrm{C} 13$ & $29(1)$ & $18(1)$ & $26(1)$ & $-9(1)$ & $12(1)$ & $-7(1)$ \\
\hline $\mathrm{C} 14$ & $12(1)$ & $13(1)$ & $21(1)$ & $-2(1)$ & $6(1)$ & $0(1)$ \\
\hline $\mathrm{C} 15$ & $34(1)$ & $19(1)$ & $23(1)$ & $-3(1)$ & $7(1)$ & $0(1)$ \\
\hline $\mathrm{C} 16$ & $24(1)$ & $28(1)$ & $42(1)$ & $-5(1)$ & $16(1)$ & $-11(1)$ \\
\hline $\mathrm{C} 17$ & $27(1)$ & $18(1)$ & $35(1)$ & $-5(1)$ & $10(1)$ & $4(1)$ \\
\hline $\mathrm{C} 18$ & $20(1)$ & $14(1)$ & $18(1)$ & $4(1)$ & $9(1)$ & $1(1)$ \\
\hline
\end{tabular}




\begin{tabular}{lllllll}
$\mathrm{C} 19$ & $22(1)$ & $20(1)$ & $18(1)$ & $2(1)$ & $3(1)$ & $2(1)$ \\
$\mathrm{C} 20$ & $32(1)$ & $25(1)$ & $29(1)$ & $4(1)$ & $21(1)$ & $-2(1)$ \\
$\mathrm{C} 21$ & $32(1)$ & $17(1)$ & $26(1)$ & $7(1)$ & $10(1)$ & $5(1)$ \\
$\mathrm{C} 22$ & $15(1)$ & $12(1)$ & $15(1)$ & $-1(1)$ & $7(1)$ & $0(1)$ \\
$\mathrm{C} 23$ & $13(1)$ & $14(1)$ & $18(1)$ & $-3(1)$ & $7(1)$ & $0(1)$ \\
$\mathrm{C} 24$ & $20(1)$ & $17(1)$ & $17(1)$ & $2(1)$ & $11(1)$ & $2(1)$ \\
\hline
\end{tabular}


Table S-19. Hydrogen coordinates (x 104) and isotropic displacement parameters $\left(\AA^{2} \times 10^{3}\right)$ for jonsc14 (2c).

\begin{tabular}{|c|c|c|c|c|}
\hline & $\mathrm{x}$ & $\mathrm{y}$ & $\mathrm{z}$ & $\mathrm{U}(\mathrm{eq})$ \\
\hline $\mathrm{H} 2$ & 12646 & 7290 & 2683 & 18 \\
\hline H3 & 13498 & 8821 & 2760 & 20 \\
\hline $\mathrm{H} 4$ & 12606 & 10370 & 2416 & 17 \\
\hline H7Aa & 7749 & 12625 & 512 & 41 \\
\hline $\mathrm{H} 7 \mathrm{Ba}$ & 8450 & 12635 & 1666 & 41 \\
\hline $\mathrm{H} 7 \mathrm{Ca}$ & 7687 & 11707 & 1159 & 41 \\
\hline H8Aa & 8200 & 11549 & -539 & 48 \\
\hline H8Ba & 7928 & 10633 & -22 & 48 \\
\hline $\mathrm{H} 8 \mathrm{Ca}$ & 8985 & 10630 & -62 & 48 \\
\hline H9Aa & 9369 & 12821 & 318 & 31 \\
\hline H9Ba & 10271 & 12015 & 837 & 31 \\
\hline $\mathrm{H} 9 \mathrm{Ca}$ & 10030 & 12817 & 1477 & 31 \\
\hline H7De & 8926 & 11415 & -506 & 27 \\
\hline $\mathrm{H} 7 \mathrm{Ee}$ & 9295 & 10367 & 80 & 27 \\
\hline $\mathrm{H} 7 \mathrm{Fe}$ & 10050 & 11322 & 384 & 27 \\
\hline H8De & 8812 & 12893 & 1360 & 33 \\
\hline H8Ee & 8805 & 12956 & 330 & 33 \\
\hline $\mathrm{H} 8 \mathrm{Fe}$ & 9851 & 12765 & 1298 & 33 \\
\hline H9De & 7541 & 11522 & 844 & 31 \\
\hline H9Ee & 7668 & 10524 & 321 & 31 \\
\hline $\mathrm{H} 9 \mathrm{Fe}$ & 7430 & 11597 & -228 & 31 \\
\hline H11A & 11081 & 10992 & 4597 & 35 \\
\hline H11B & 11491 & 10727 & 3838 & 35 \\
\hline $\mathrm{H} 11 \mathrm{C}$ & 10656 & 10014 & 3911 & 35 \\
\hline $\mathrm{H} 12 \mathrm{~A}$ & 9409 & 11615 & 4099 & 43 \\
\hline H12B & 8757 & 10836 & 3240 & 43 \\
\hline $\mathrm{H} 12 \mathrm{C}$ & 8701 & 12032 & 3023 & 43 \\
\hline $\mathrm{H} 13 \mathrm{~A}$ & 10785 & 12690 & 3896 & 36 \\
\hline H13B & 9983 & 12897 & 2784 & 36 \\
\hline $\mathrm{H} 13 \mathrm{C}$ & 11056 & 12377 & 3049 & 36 \\
\hline
\end{tabular}




\begin{tabular}{lrrrr} 
H15A & 8255 & 5927 & -223 & 41 \\
H15B & 9108 & 6796 & 155 & 41 \\
H15C & 8053 & 6981 & 174 & 41 \\
H16A & 7791 & 5055 & 884 & 47 \\
H16B & 7684 & 6076 & 1390 & 47 \\
H16C & 8438 & 5193 & 2036 & 47 \\
H17A & 9442 & 4700 & 822 & 42 \\
H17B & 10067 & 4866 & 1973 & 42 \\
H17C & 10344 & 5517 & 1259 & 42 \\
H19A & 11008 & 7138 & 4823 & 33 \\
H19B & 10627 & 7992 & 3994 & 33 \\
H19C & 11483 & 7194 & 4089 & 33 \\
H20A & 9378 & 6444 & 4374 & 39 \\
H20B & 8668 & 5982 & 3318 & 39 \\
H20C & 8759 & 7189 & 3474 & 39 \\
H21A & 10800 & 5334 & 4342 & 39 \\
H21B & 11054 & 5475 & 3456 & 39 \\
H21C & 9993 & 4985 & 3270 & 39 \\
& & & & \\
\hline
\end{tabular}


Table S-20. Torsion angles $\left[{ }^{\circ}\right]$ for jonsc14 (2c).

\begin{tabular}{|c|c|c|c|}
\hline C14-P1-O1-C1 & $143.01(13)$ & C6'e-P2-C6a-C7a & $122(2)$ \\
\hline C18-P1-O1-C1 & $-106.61(13)$ & C10-P2-C6a-C7a & $-54.19(18)$ \\
\hline W1-P1-O1-C1 & $14.55(13)$ & W1-P2-C6a-C7a & $94.10(16)$ \\
\hline C6a-P2-O2-C5 & $-144.91(13)$ & O2-P2-C6'e-C8'e & $-82.2(7)$ \\
\hline C6'e-P2-O2-C5 & $-136.4(3)$ & C6a-P2-C6'e-C8'e & $15.4(19)$ \\
\hline $\mathrm{C} 10-\mathrm{P} 2-\mathrm{O} 2-\mathrm{C} 5$ & $105.89(12)$ & C10-P2-C6'e-C8'e & 19.1(7) \\
\hline W1-P2-O2-C5 & $-15.66(12)$ & W1-P2-C6'e-C8'e & $169.6(6)$ \\
\hline P1-O1-C1-N1 & $-13.1(2)$ & O2-P2-C6'e-C9'e & $156.0(5)$ \\
\hline $\mathrm{P} 1-\mathrm{O} 1-\mathrm{C} 1-\mathrm{C} 2$ & $167.69(12)$ & C6a-P2-C6'e-C9'e & $-106(2)$ \\
\hline C5-N1-C1-O1 & $-174.13(14)$ & C10-P2-C6'e-C9'e & $-102.7(6)$ \\
\hline W1-N1-C1-O1 & $3.33(19)$ & W1-P2-C6'e-C9'e & $47.7(6)$ \\
\hline C5-N1-C1-C2 & $5.0(2)$ & O2-P2-C6'e-C7'e & $37.5(6)$ \\
\hline W1-N1-C1-C2 & $-177.50(12)$ & C6a-P2-C6'e-C7'e & $135(3)$ \\
\hline $\mathrm{O} 1-\mathrm{C} 1-\mathrm{C} 2-\mathrm{C} 3$ & $176.29(16)$ & C10-P2-C6'e-C7'e & $138.7(5)$ \\
\hline $\mathrm{N} 1-\mathrm{C} 1-\mathrm{C} 2-\mathrm{C} 3$ & $-2.9(3)$ & W1-P2-C6'e-C7'e & $-70.8(6)$ \\
\hline $\mathrm{C} 1-\mathrm{C} 2-\mathrm{C} 3-\mathrm{C} 4$ & $-0.7(3)$ & $\mathrm{O} 2-\mathrm{P} 2-\mathrm{C} 10-\mathrm{C} 12$ & $-175.37(14)$ \\
\hline $\mathrm{C} 2-\mathrm{C} 3-\mathrm{C} 4-\mathrm{C} 5$ & $1.8(3)$ & C6a-P2-C10-C12 & $83.72(16)$ \\
\hline $\mathrm{P} 2-\mathrm{O} 2-\mathrm{C} 5-\mathrm{N} 1$ & $12.87(19)$ & C6'e-P2-C10-C12 & $83.2(3)$ \\
\hline $\mathrm{P} 2-\mathrm{O} 2-\mathrm{C} 5-\mathrm{C} 4$ & $-167.54(12)$ & W1-P2-C10-C12 & $-67.05(15)$ \\
\hline $\mathrm{C} 1-\mathrm{N} 1-\mathrm{C} 5-\mathrm{O} 2$ & $175.75(13)$ & $\mathrm{O} 2-\mathrm{P} 2-\mathrm{C} 10-\mathrm{C} 13$ & $60.83(15)$ \\
\hline W1-N1-C5-O2 & $-1.71(19)$ & C6a-P2-C10-C13 & $-40.08(17)$ \\
\hline C1-N1-C5-C4 & $-3.8(2)$ & C6'e-P2-C10-C13 & $-40.7(4)$ \\
\hline W1-N1-C5-C4 & $178.71(12)$ & W1-P2-C10-C13 & $169.14(12)$ \\
\hline $\mathrm{C} 3-\mathrm{C} 4-\mathrm{C} 5-\mathrm{O} 2$ & $-179.07(15)$ & O2-P2-C10-C11 & $-56.14(13)$ \\
\hline $\mathrm{C} 3-\mathrm{C} 4-\mathrm{C} 5-\mathrm{N} 1$ & $0.5(2)$ & C6a-P2-C10-C11 & $-157.05(13)$ \\
\hline O2-P2-C6a-C8a & $88.9(2)$ & C6'e-P2-C10-C11 & $-157.6(3)$ \\
\hline C6'e-P2-C6a-C8a & $5(2)$ & W1-P2-C10-C11 & $52.17(14)$ \\
\hline C10-P2-C6a-C8a & $-171.18(19)$ & O1-P1-C14-C15 & $-86.71(14)$ \\
\hline W1-P2-C6a-C8a & $-22.9(2)$ & C18-P1-C14-C15 & $172.97(14)$ \\
\hline O2-P2-C6a-C9a & $-29.78(18)$ & W1-P1-C14-C15 & $24.10(16)$ \\
\hline C6'e-P2-C6a-C9a & $-113(2)$ & O1-P1-C14-C16 & $155.56(14)$ \\
\hline C10-P2-C6a-C9a & $70.14(18)$ & C18-P1-C14-C16 & $55.24(15)$ \\
\hline W1-P2-C6a-C9a & $-141.56(14)$ & W1-P1-C14-C16 & $-93.63(14)$ \\
\hline O2-P2-C6a-C7a & $-154.12(16)$ & O1-P1-C14-C17 & $32.74(15)$ \\
\hline
\end{tabular}




$\begin{array}{lclc}\text { C18-P1-C14-C17 } & -67.59(16) & \text { C14-P1-C18-C21 } & 39.98(16) \\ \text { W1-P1-C14-C17 } & 143.55(12) & \text { W1-P1-C18-C21 } & -168.84(12) \\ \text { O1-P1-C18-C20 } & 175.13(13) & \text { O1-P1-C18-C19 } & 56.13(13) \\ \text { C14-P1-C18-C20 } & -83.81(14) & \text { C14-P1-C18-C19 } & 157.19(12) \\ \text { W1-P1-C18-C20 } & 67.37(14) & \text { W1-P1-C18-C19 } & -51.63(13) \\ \text { O1-P1-C18-C21 } & -61.08(15) & & \end{array}$


REFERENCE NUMBER: jonlm21 (2d)

CRYSTAL STRUCTURE REPORT

$\mathrm{C}_{20} \mathrm{H}_{31} \mathrm{~N} \mathrm{O}_{5} \mathrm{P}_{2} \mathrm{~W}$

or

(iPrPONOP)W(CO)

Report prepared for:

L. Munjanja, Prof. W. Jones

May 21, 2015

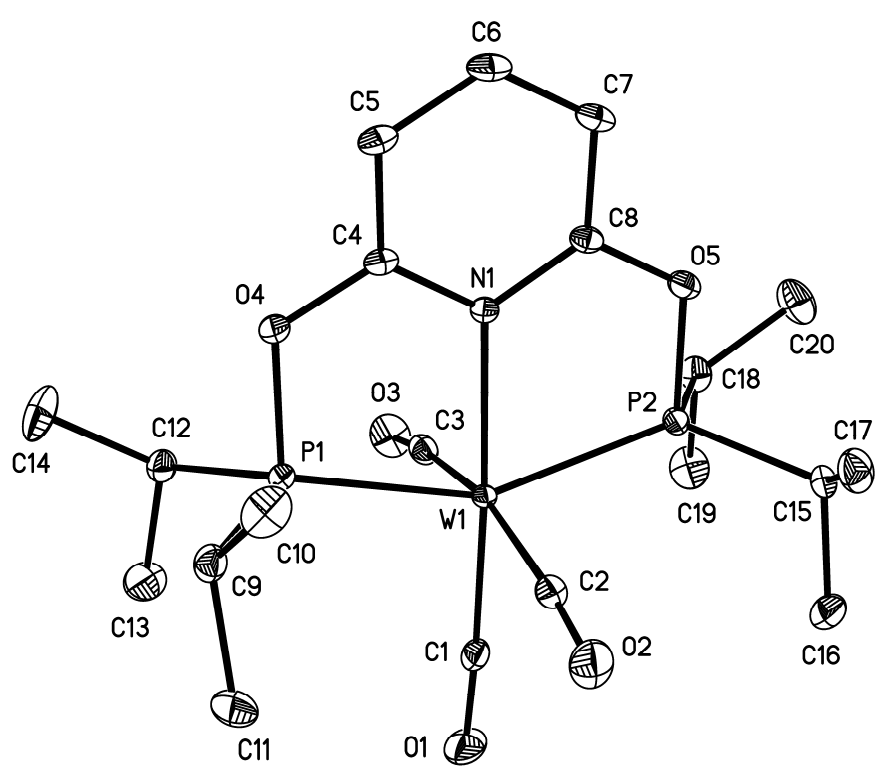

William W. Brennessel

X-ray Crystallographic Facility

Department of Chemistry, University of Rochester

120 Trustee Road

Rochester, NY 14627 


\section{Data collection}

A crystal $\left(0.40 \times 0.36 \times 0.36 \mathrm{~mm}^{3}\right)$ was placed onto the tip of a thin glass optical fiber and mounted on a Bruker SMART APEX II CCD platform diffractometer for a data collection at 100.0(5) K. ${ }^{1}$ A preliminary set of cell constants and an orientation matrix were calculated from reflections harvested from three orthogonal wedges of reciprocal space. The full data collection was carried out using MoK $\alpha$ radiation (graphite monochromator) with a frame time of 10 seconds and a detector distance of $4.03 \mathrm{~cm}$. A randomly oriented region of reciprocal space was surveyed: six major sections of frames were collected with $0.50^{\circ}$ steps in $\omega$ at six different $\phi$ settings and a detector position of $-38^{\circ}$ in $2 \theta$. The intensity data were corrected for absorption. ${ }^{2}$ Final cell constants were calculated from the xyz centroids of 3911 strong reflections from the actual data collection after integration. ${ }^{3}$ See Table S19 for additional crystal and refinement information.

\section{Structure solution and refinement}

The structure was solved using SIR20114 and refined using SHELXL-2014/7. ${ }^{5}$ The space group $P 2{ }_{1} 2_{1} 2_{1}$ was determined based on systematic absences. A direct-methods solution was calculated which provided most nonhydrogen atoms from the E-map. Full-matrix least squares / difference Fourier cycles were performed which located the remaining non-hydrogen atoms. All non-hydrogen atoms were refined with anisotropic displacement parameters. All hydrogen atoms were placed in ideal positions and refined as riding atoms with relative isotropic displacement parameters. The final full matrix least squares refinement converged to $R 1=0.0172\left(F^{2}, I>2 \sigma(I)\right)$ and $w R 2=0.0363\left(F^{2}\right.$, all data $)$.

\section{Structure description}

The structure is similar to the one suggested. The asymmetric unit contains one molecule in a general position. The structure is a second polymorph of the previously determined one (local code JONSC16).

Unless noted otherwise all structural diagrams containing thermal displacement ellipsoids are drawn at the $50 \%$ probability level.

Data collection, structure solution, and structure refinement were conducted at the X-ray Crystallographic Facility, B51 Hutchison Hall, Department of Chemistry, University of Rochester. All publications arising from this report MUST either 1) include William W. Brennessel as a coauthor or 2) acknowledge William W. Brennessel and the Xray Crystallographic Facility of the Department of Chemistry at the University of Rochester. 
1 APEX2, version 2014.11-0; Bruker AXS: Madison, WI, 2014.

2 Sheldrick, G. M. SADABS, version 2014/5; University of Göttingen: Göttingen, Germany, 2014.

3 SAINT, version 8.32B; Bruker AXS: Madison, WI, 2014.

4 Burla, M. C.; Caliandro, R.; Camalli, M.; Carrozzini, B.; Cascarano, G. L.; Giacovazzo, C.; Mallamo, M.; Mazzone, A.; Polidori, G.; Spagna, R. SIR2011: a new package for crystal structure determination and refinement, version 1.0; Istituto di Cristallografia: Bari, Italy, 2012.

5 Sheldrick, G. M. SHELXL-2014/7; University of Göttingen: Göttingen, Germany, 2014.

Some equations of interest:

$$
\begin{gathered}
R_{\mathrm{int}}=\Sigma\left|F_{\mathrm{o}}^{2}-<F_{\mathrm{o}}^{2}>\right| / \Sigma\left|F_{\mathrm{o}}{ }^{2}\right| \\
R 1=\Sigma|| F_{\mathrm{o}}|-| F_{\mathrm{c}} \| / \Sigma\left|F_{\mathrm{o}}\right| \\
w R 2=\left[\Sigma\left[w\left(F_{\mathrm{o}}{ }^{2}-F_{\mathrm{c}}{ }^{2}\right)^{2}\right] / \Sigma\left[w\left(F_{\mathrm{o}}{ }^{2}\right)^{2}\right]\right]^{1 / 2} \\
\text { where } w=1 /\left[\sigma^{2}\left(F_{\mathrm{o}}{ }^{2}\right)+(a P)^{2}+b P\right] \text { and } \\
P=1 / 3 \max \left(0, F_{\mathrm{o}}{ }^{2}\right)+2 / 3 F_{\mathrm{c}}{ }^{2} \\
\mathrm{GOF}=S=\left[\Sigma\left[w\left(F_{\mathrm{o}}{ }^{2}-F_{\mathrm{c}}{ }^{2}\right)^{2}\right] /(m-n)\right]^{1 / 2}
\end{gathered}
$$

where $m=$ number of reflections and $n=$ number of parameters

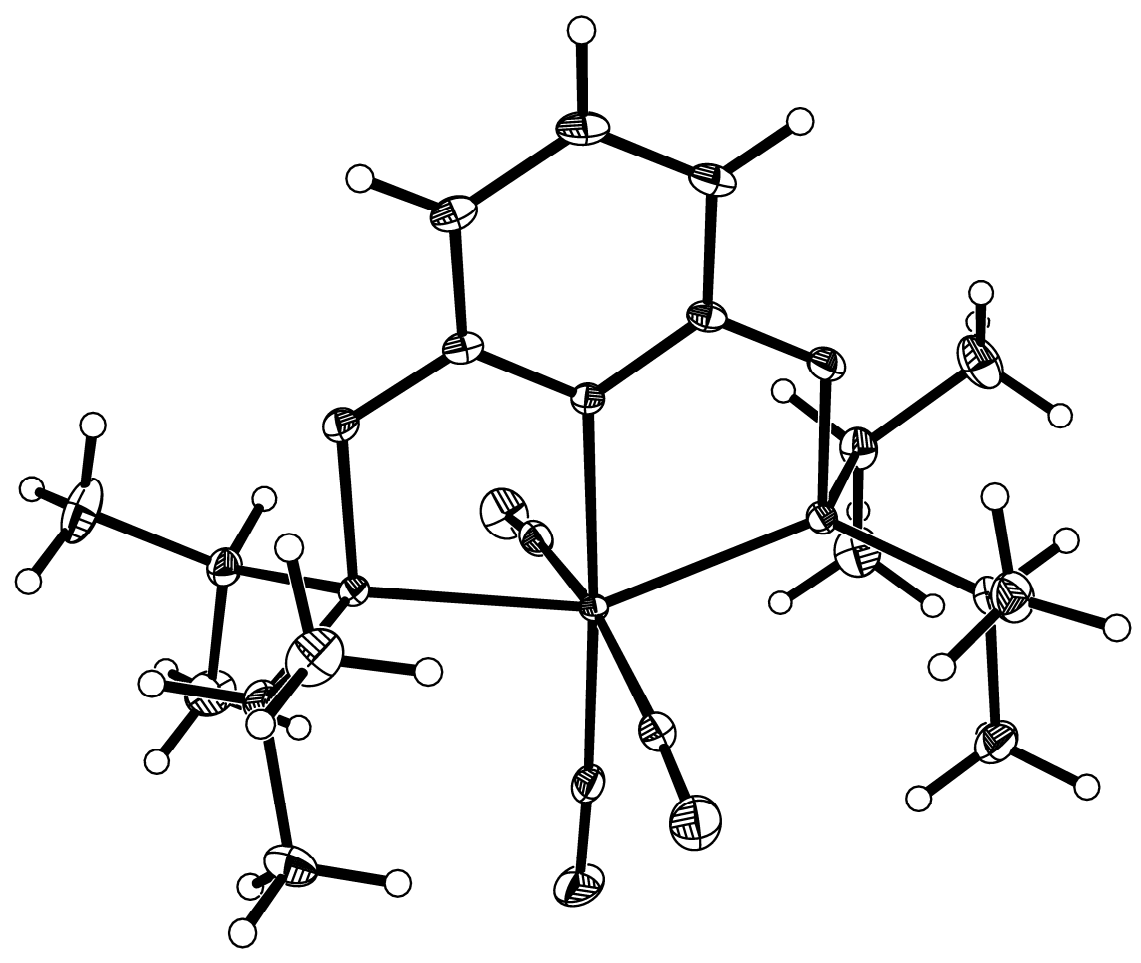


Table S-21. Crystal data and structure refinement for jonlm21 (2d).

\begin{tabular}{|c|c|c|}
\hline Identification code & \multicolumn{2}{|l|}{ jonlm21 } \\
\hline Empirical formula & \multicolumn{2}{|c|}{ C20 H31 N O5 P2 W } \\
\hline Formula weight & \multicolumn{2}{|l|}{611.25} \\
\hline Temperature & \multicolumn{2}{|l|}{$100.0(5) \mathrm{K}$} \\
\hline Wavelength & \multicolumn{2}{|l|}{$0.71073 \AA$} \\
\hline Crystal system & \multicolumn{2}{|l|}{ orthorhombic } \\
\hline Space group & \multicolumn{2}{|l|}{$P 2_{1} 2_{1} 2_{1}$} \\
\hline \multirow[t]{3}{*}{ Unit cell dimensions } & $a=10.7399(19) \AA$ & $\alpha=90^{\circ}$ \\
\hline & $b=11.323(2) \AA$ & $\beta=90^{\circ}$ \\
\hline & $c=19.424(4) \AA$ & $\gamma=90^{\circ}$ \\
\hline Volume & \multicolumn{2}{|l|}{$2362.1(7) \AA^{3}$} \\
\hline$Z$ & \multicolumn{2}{|l|}{4} \\
\hline Density (calculated) & \multicolumn{2}{|l|}{$1.719 \mathrm{Mg} / \mathrm{m}^{3}$} \\
\hline Absorption coefficient & \multicolumn{2}{|l|}{$5.055 \mathrm{~mm}^{-1}$} \\
\hline$F(000)$ & \multicolumn{2}{|l|}{1208} \\
\hline Crystal color, morphology & \multicolumn{2}{|l|}{ yellow, block } \\
\hline Crystal size & \multicolumn{2}{|c|}{$0.40 \times 0.36 \times 0.36 \mathrm{~mm}^{3}$} \\
\hline Theta range for data collection & \multicolumn{2}{|l|}{2.082 to $38.588^{\circ}$} \\
\hline Index ranges & \multicolumn{2}{|c|}{$-18 \leq h \leq 18,-19 \leq k \leq 19,-34 \leq l \leq 33$} \\
\hline Reflections collected & \multicolumn{2}{|l|}{73539} \\
\hline Independent reflections & \multicolumn{2}{|c|}{$12971[R($ int $)=0.0356]$} \\
\hline Observed reflections & \multicolumn{2}{|l|}{12495} \\
\hline Completeness to theta $=37.785^{\circ}$ & \multicolumn{2}{|l|}{$99.9 \%$} \\
\hline Absorption correction & \multicolumn{2}{|l|}{ Multi-scan } \\
\hline Max. and min. transmission & \multicolumn{2}{|l|}{0.4404 and 0.2668} \\
\hline Refinement method & \multicolumn{2}{|c|}{ Full-matrix least-squares on $F^{2}$} \\
\hline Data / restraints / parameters & \multicolumn{2}{|l|}{$12971 / 0 / 270$} \\
\hline Goodness-of-fit on $F^{2}$ & \multicolumn{2}{|l|}{1.079} \\
\hline Final $R$ indices $[I>2 \operatorname{sigma}(I)]$ & \multicolumn{2}{|c|}{$R 1=0.0172, w R 2=0.0360$} \\
\hline$R$ indices (all data) & \multicolumn{2}{|c|}{$R 1=0.0187, w R 2=0.0363$} \\
\hline Absolute structure parameter & \multicolumn{2}{|l|}{$0.0091(19)$} \\
\hline Largest diff. peak and hole & \multicolumn{2}{|c|}{0.866 and -1.165 e. $\AA^{-3}$} \\
\hline
\end{tabular}


Table S-22. Atomic coordinates (x $\left.10^{4}\right)$ and equivalent isotropic displacement parameters $\left(\AA^{2} \mathrm{x} 10^{3}\right)$ for jonlm21 (2d). $U_{\text {eq }}$ is defined as one third of the trace of the orthogonalized $U_{i j}$ tensor.

\begin{tabular}{|c|c|c|c|c|}
\hline & $\mathrm{x}$ & $\mathrm{y}$ & $\mathrm{z}$ & $\mathrm{U}_{\mathrm{eq}}$ \\
\hline W1 & $2297(1)$ & $4779(1)$ & $8863(1)$ & $8(1)$ \\
\hline P1 & $2689(1)$ & $2805(1)$ & 8501(1) & $10(1)$ \\
\hline $\mathrm{P} 2$ & $943(1)$ & $6428(1)$ & $9080(1)$ & $10(1)$ \\
\hline $\mathrm{O} 1$ & $5046(2)$ & $5581(2)$ & $9125(1)$ & $25(1)$ \\
\hline $\mathrm{O} 2$ & $2603(2)$ & $4213(2)$ & 10451(1) & $25(1)$ \\
\hline $\mathrm{O} 3$ & $2913(2)$ & $5640(2)$ & $7348(1)$ & $23(1)$ \\
\hline $\mathrm{O} 4$ & $1253(1)$ & 2281(1) & $8309(1)$ & $13(1)$ \\
\hline $\mathrm{O} 5$ & $-505(1)$ & $5842(1)$ & 8992(1) & $14(1)$ \\
\hline N1 & $395(2)$ & $4053(2)$ & $8675(1)$ & $10(1)$ \\
\hline $\mathrm{C} 1$ & $4014(2)$ & $5317(2)$ & $9030(1)$ & $14(1)$ \\
\hline $\mathrm{C} 2$ & $2403(2)$ & $4384(2)$ & $9875(1)$ & $14(1)$ \\
\hline $\mathrm{C} 3$ & $2614(2)$ & $5316(2)$ & $7889(1)$ & $13(1)$ \\
\hline $\mathrm{C} 4$ & $242(2)$ & $2920(2)$ & $8471(1)$ & $11(1)$ \\
\hline $\mathrm{C} 5$ & $-924(2)$ & $2390(2)$ & $8416(1)$ & $14(1)$ \\
\hline C6 & $-1958(2)$ & $3068(2)$ & $8552(1)$ & $15(1)$ \\
\hline $\mathrm{C} 7$ & $-1839(2)$ & $4244(2)$ & $8736(1)$ & $14(1)$ \\
\hline $\mathrm{C} 8$ & $-638(2)$ & $4698(2)$ & $8796(1)$ & $11(1)$ \\
\hline $\mathrm{C} 9$ & $3221(2)$ & $1608(2)$ & $9077(1)$ & $15(1)$ \\
\hline $\mathrm{C} 10$ & 2189(3) & $1260(2)$ & $9577(1)$ & $22(1)$ \\
\hline $\mathrm{C} 11$ & $4399(2)$ & $1995(2)$ & $9465(1)$ & $22(1)$ \\
\hline $\mathrm{C} 12$ & $3430(2)$ & $2517(2)$ & 7663(1) & $14(1)$ \\
\hline $\mathrm{C} 13$ & $4798(2)$ & $2868(3)$ & $7675(1)$ & $26(1)$ \\
\hline $\mathrm{C} 14$ & $3252(3)$ & $1263(2)$ & 7384(1) & $28(1)$ \\
\hline $\mathrm{C} 15$ & $749(2)$ & $7159(2)$ & 9921(1) & $14(1)$ \\
\hline $\mathrm{C} 16$ & $2017(2)$ & $7625(2)$ & 10161(1) & $22(1)$ \\
\hline $\mathrm{C} 17$ & $187(2)$ & $6339(2)$ & $10465(1)$ & $20(1)$ \\
\hline $\mathrm{C} 18$ & $777(2)$ & $7608(2)$ & $8433(1)$ & $16(1)$ \\
\hline C19 & 1976(3) & $8326(2)$ & $8353(1)$ & $23(1)$ \\
\hline $\mathrm{C} 20$ & $-360(3)$ & $8401(2)$ & $8515(1)$ & $25(1)$ \\
\hline
\end{tabular}


Table S-23. Bond lengths $[\AA]$ and angles $\left[{ }^{\circ}\right]$ for jonlm21 (2d).

\begin{tabular}{|c|c|c|c|}
\hline $\mathrm{W}(1)-\mathrm{C}(1)$ & $1.969(2)$ & $\mathrm{C}(11)-\mathrm{H}(11 \mathrm{C})$ & 0.9800 \\
\hline $\mathrm{W}(1)-\mathrm{C}(3)$ & $2.016(2)$ & $\mathrm{C}(12)-\mathrm{C}(13)$ & $1.522(3)$ \\
\hline $\mathrm{W}(1)-\mathrm{C}(2)$ & $2.019(2)$ & $C(12)-C(14)$ & $1.532(3)$ \\
\hline $\mathrm{W}(1)-\mathrm{N}(1)$ & $2.2318(17)$ & $\mathrm{C}(12)-\mathrm{H}(12)$ & 1.0000 \\
\hline $\mathrm{W}(1)-\mathrm{P}(1)$ & $2.3801(6)$ & $\mathrm{C}(13)-\mathrm{H}(13 \mathrm{~A})$ & 0.9800 \\
\hline $\mathrm{W}(1)-\mathrm{P}(2)$ & $2.4038(6)$ & $\mathrm{C}(13)-\mathrm{H}(13 \mathrm{~B})$ & 0.9800 \\
\hline $\mathrm{P}(1)-\mathrm{O}(4)$ & $1.6939(16)$ & $\mathrm{C}(13)-\mathrm{H}(13 \mathrm{C})$ & 0.9800 \\
\hline $\mathrm{P}(1)-\mathrm{C}(12)$ & $1.841(2)$ & $\mathrm{C}(14)-\mathrm{H}(14 \mathrm{~A})$ & 0.9800 \\
\hline $\mathrm{P}(1)-\mathrm{C}(9)$ & $1.848(2)$ & $\mathrm{C}(14)-\mathrm{H}(14 \mathrm{~B})$ & 0.9800 \\
\hline $\mathrm{P}(2)-\mathrm{O}(5)$ & $1.6997(17)$ & $\mathrm{C}(14)-\mathrm{H}(14 \mathrm{C})$ & 0.9800 \\
\hline $\mathrm{P}(2)-\mathrm{C}(18)$ & $1.843(2)$ & $C(15)-C(17)$ & $1.530(3)$ \\
\hline $\mathrm{P}(2)-\mathrm{C}(15)$ & $1.844(2)$ & $C(15)-C(16)$ & $1.533(3)$ \\
\hline $\mathrm{O}(1)-\mathrm{C}(1)$ & $1.163(3)$ & $\mathrm{C}(15)-\mathrm{H}(15)$ & 1.0000 \\
\hline $\mathrm{O}(2)-\mathrm{C}(2)$ & $1.157(2)$ & $\mathrm{C}(16)-\mathrm{H}(16 \mathrm{~A})$ & 0.9800 \\
\hline $\mathrm{O}(3)-\mathrm{C}(3)$ & $1.157(3)$ & $\mathrm{C}(16)-\mathrm{H}(16 \mathrm{~B})$ & 0.9800 \\
\hline $\mathrm{O}(4)-\mathrm{C}(4)$ & $1.343(3)$ & $\mathrm{C}(16)-\mathrm{H}(16 \mathrm{C})$ & 0.9800 \\
\hline $\mathrm{O}(5)-\mathrm{C}(8)$ & $1.357(3)$ & $\mathrm{C}(17)-\mathrm{H}(17 \mathrm{~A})$ & 0.9800 \\
\hline $\mathrm{N}(1)-\mathrm{C}(8)$ & $1.349(3)$ & $\mathrm{C}(17)-\mathrm{H}(17 \mathrm{~B})$ & 0.9800 \\
\hline $\mathrm{N}(1)-\mathrm{C}(4)$ & $1.353(3)$ & $\mathrm{C}(17)-\mathrm{H}(17 \mathrm{C})$ & 0.9800 \\
\hline$C(4)-C(5)$ & $1.392(3)$ & $C(18)-C(20)$ & $1.523(3)$ \\
\hline$C(5)-C(6)$ & $1.376(3)$ & $\mathrm{C}(18)-\mathrm{C}(19)$ & $1.531(3)$ \\
\hline $\mathrm{C}(5)-\mathrm{H}(5)$ & 0.9500 & $\mathrm{C}(18)-\mathrm{H}(18)$ & 1.0000 \\
\hline$C(6)-C(7)$ & $1.384(3)$ & C(19)-H(19A) & 0.9800 \\
\hline $\mathrm{C}(6)-\mathrm{H}(6)$ & 0.9500 & C(19)-H(19B) & 0.9800 \\
\hline$C(7)-C(8)$ & $1.394(3)$ & C(19)-H(19C) & 0.9800 \\
\hline $\mathrm{C}(7)-\mathrm{H}(7)$ & 0.9500 & $\mathrm{C}(20)-\mathrm{H}(20 \mathrm{~A})$ & 0.9800 \\
\hline C(9)-C(10) & $1.525(3)$ & $\mathrm{C}(20)-\mathrm{H}(20 \mathrm{~B})$ & 0.9800 \\
\hline $\mathrm{C}(9)-\mathrm{C}(11)$ & $1.537(3)$ & $\mathrm{C}(20)-\mathrm{H}(20 \mathrm{C})$ & 0.9800 \\
\hline C(9)-H(9) & 1.0000 & $C(1)-W(1)-C(3)$ & $84.46(9)$ \\
\hline $\mathrm{C}(10)-\mathrm{H}(10 \mathrm{~A})$ & 0.9800 & $C(1)-W(1)-C(2)$ & $81.68(9)$ \\
\hline $\mathrm{C}(10)-\mathrm{H}(10 \mathrm{~B})$ & 0.9800 & $C(3)-W(1)-C(2)$ & $166.14(9)$ \\
\hline $\mathrm{C}(10)-\mathrm{H}(10 \mathrm{C})$ & 0.9800 & $\mathrm{C}(1)-\mathrm{W}(1)-\mathrm{N}(1)$ & $176.42(8)$ \\
\hline $\mathrm{C}(11)-\mathrm{H}(11 \mathrm{~A})$ & 0.9800 & $\mathrm{C}(3)-\mathrm{W}(1)-\mathrm{N}(1)$ & $96.43(7)$ \\
\hline C(11)-H(11B) & 0.9800 & $\mathrm{C}(2)-\mathrm{W}(1)-\mathrm{N}(1)$ & $97.42(8)$ \\
\hline
\end{tabular}




\begin{tabular}{|c|c|c|c|}
\hline $\mathrm{C}(1)-\mathrm{W}(1)-\mathrm{P}(1)$ & $100.01(7)$ & $\mathrm{C}(5)-\mathrm{C}(6)-\mathrm{H}(6)$ & 119.6 \\
\hline C(3)-W(1)-P(1) & $88.67(6)$ & $\mathrm{C}(7)-\mathrm{C}(6)-\mathrm{H}(6)$ & 119.6 \\
\hline $\mathrm{C}(2)-\mathrm{W}(1)-\mathrm{P}(1)$ & $93.97(6)$ & $\mathrm{C}(6)-\mathrm{C}(7)-\mathrm{C}(8)$ & $117.5(2)$ \\
\hline $\mathrm{N}(1)-\mathrm{W}(1)-\mathrm{P}(1)$ & $76.57(5)$ & $\mathrm{C}(6)-\mathrm{C}(7)-\mathrm{H}(7)$ & 121.2 \\
\hline C(1)-W(1)-P(2) & $107.29(7)$ & $\mathrm{C}(8)-\mathrm{C}(7)-\mathrm{H}(7)$ & 121.2 \\
\hline C(3)-W(1)-P(2) & $91.85(6)$ & $\mathrm{N}(1)-\mathrm{C}(8)-\mathrm{O}(5)$ & $118.60(17)$ \\
\hline C(2)-W(1)-P(2) & $92.02(6)$ & $\mathrm{N}(1)-\mathrm{C}(8)-\mathrm{C}(7)$ & $123.2(2)$ \\
\hline $\mathrm{N}(1)-\mathrm{W}(1)-\mathrm{P}(2)$ & $76.17(5)$ & $\mathrm{O}(5)-\mathrm{C}(8)-\mathrm{C}(7)$ & $118.22(18)$ \\
\hline $\mathrm{P}(1)-\mathrm{W}(1)-\mathrm{P}(2)$ & $152.62(2)$ & $\mathrm{C}(10)-\mathrm{C}(9)-\mathrm{C}(11)$ & 111.1(2) \\
\hline $\mathrm{O}(4)-\mathrm{P}(1)-\mathrm{C}(12)$ & $97.85(9)$ & $\mathrm{C}(10)-\mathrm{C}(9)-\mathrm{P}(1)$ & $110.49(17)$ \\
\hline $\mathrm{O}(4)-\mathrm{P}(1)-\mathrm{C}(9)$ & $99.11(10)$ & $\mathrm{C}(11)-\mathrm{C}(9)-\mathrm{P}(1)$ & $110.04(17)$ \\
\hline $\mathrm{C}(12)-\mathrm{P}(1)-\mathrm{C}(9)$ & $105.76(10)$ & $\mathrm{C}(10)-\mathrm{C}(9)-\mathrm{H}(9)$ & 108.4 \\
\hline $\mathrm{O}(4)-\mathrm{P}(1)-\mathrm{W}(1)$ & $103.49(6)$ & $\mathrm{C}(11)-\mathrm{C}(9)-\mathrm{H}(9)$ & 108.4 \\
\hline $\mathrm{C}(12)-\mathrm{P}(1)-\mathrm{W}(1)$ & $120.21(8)$ & $\mathrm{P}(1)-\mathrm{C}(9)-\mathrm{H}(9)$ & 108.4 \\
\hline C(9)-P(1)-W(1) & $124.39(7)$ & $\mathrm{C}(9)-\mathrm{C}(10)-\mathrm{H}(10 \mathrm{~A})$ & 109.5 \\
\hline $\mathrm{O}(5)-\mathrm{P}(2)-\mathrm{C}(18)$ & $97.25(10)$ & $\mathrm{C}(9)-\mathrm{C}(10)-\mathrm{H}(10 \mathrm{~B})$ & 109.5 \\
\hline $\mathrm{O}(5)-\mathrm{P}(2)-\mathrm{C}(15)$ & $99.25(9)$ & $\mathrm{H}(10 \mathrm{~A})-\mathrm{C}(10)-\mathrm{H}(10 \mathrm{~B})$ & 109.5 \\
\hline$C(18)-P(2)-C(15)$ & $105.50(11)$ & $\mathrm{C}(9)-\mathrm{C}(10)-\mathrm{H}(10 \mathrm{C})$ & 109.5 \\
\hline $\mathrm{O}(5)-\mathrm{P}(2)-\mathrm{W}(1)$ & $103.47(6)$ & $\mathrm{H}(10 \mathrm{~A})-\mathrm{C}(10)-\mathrm{H}(10 \mathrm{C})$ & 109.5 \\
\hline C(18)-P(2)-W(1) & $120.18(8)$ & $\mathrm{H}(10 \mathrm{~B})-\mathrm{C}(10)-\mathrm{H}(10 \mathrm{C})$ & 109.5 \\
\hline $\mathrm{C}(15)-\mathrm{P}(2)-\mathrm{W}(1)$ & $124.93(7)$ & $\mathrm{C}(9)-\mathrm{C}(11)-\mathrm{H}(11 \mathrm{~A})$ & 109.5 \\
\hline $\mathrm{C}(4)-\mathrm{O}(4)-\mathrm{P}(1)$ & $119.74(13)$ & $\mathrm{C}(9)-\mathrm{C}(11)-\mathrm{H}(11 \mathrm{~B})$ & 109.5 \\
\hline $\mathrm{C}(8)-\mathrm{O}(5)-\mathrm{P}(2)$ & $119.79(13)$ & $\mathrm{H}(11 \mathrm{~A})-\mathrm{C}(11)-\mathrm{H}(11 \mathrm{~B})$ & 109.5 \\
\hline $\mathrm{C}(8)-\mathrm{N}(1)-\mathrm{C}(4)$ & $117.64(17)$ & $\mathrm{C}(9)-\mathrm{C}(11)-\mathrm{H}(11 \mathrm{C})$ & 109.5 \\
\hline $\mathrm{C}(8)-\mathrm{N}(1)-\mathrm{W}(1)$ & $121.67(13)$ & $\mathrm{H}(11 \mathrm{~A})-\mathrm{C}(11)-\mathrm{H}(11 \mathrm{C})$ & 109.5 \\
\hline $\mathrm{C}(4)-\mathrm{N}(1)-\mathrm{W}(1)$ & $120.58(14)$ & $\mathrm{H}(11 \mathrm{~B})-\mathrm{C}(11)-\mathrm{H}(11 \mathrm{C})$ & 109.5 \\
\hline $\mathrm{O}(1)-\mathrm{C}(1)-\mathrm{W}(1)$ & $176.8(2)$ & $\mathrm{C}(13)-\mathrm{C}(12)-\mathrm{C}(14)$ & $111.5(2)$ \\
\hline $\mathrm{O}(2)-\mathrm{C}(2)-\mathrm{W}(1)$ & $172.0(2)$ & $\mathrm{C}(13)-\mathrm{C}(12)-\mathrm{P}(1)$ & $111.01(15)$ \\
\hline $\mathrm{O}(3)-\mathrm{C}(3)-\mathrm{W}(1)$ & $173.4(2)$ & $\mathrm{C}(14)-\mathrm{C}(12)-\mathrm{P}(1)$ & $115.04(17)$ \\
\hline $\mathrm{O}(4)-\mathrm{C}(4)-\mathrm{N}(1)$ & $118.74(18)$ & $\mathrm{C}(13)-\mathrm{C}(12)-\mathrm{H}(12)$ & 106.2 \\
\hline $\mathrm{O}(4)-\mathrm{C}(4)-\mathrm{C}(5)$ & $118.52(19)$ & $\mathrm{C}(14)-\mathrm{C}(12)-\mathrm{H}(12)$ & 106.2 \\
\hline $\mathrm{N}(1)-\mathrm{C}(4)-\mathrm{C}(5)$ & $122.7(2)$ & $\mathrm{P}(1)-\mathrm{C}(12)-\mathrm{H}(12)$ & 106.2 \\
\hline$C(6)-C(5)-C(4)$ & $118.1(2)$ & $\mathrm{C}(12)-\mathrm{C}(13)-\mathrm{H}(13 \mathrm{~A})$ & 109.5 \\
\hline $\mathrm{C}(6)-\mathrm{C}(5)-\mathrm{H}(5)$ & 121.0 & $\mathrm{C}(12)-\mathrm{C}(13)-\mathrm{H}(13 \mathrm{~B})$ & 109.5 \\
\hline $\mathrm{C}(4)-\mathrm{C}(5)-\mathrm{H}(5)$ & 121.0 & $\mathrm{H}(13 \mathrm{~A})-\mathrm{C}(13)-\mathrm{H}(13 \mathrm{~B})$ & 109.5 \\
\hline$C(5)-C(6)-C(7)$ & $120.8(2)$ & $\mathrm{C}(12)-\mathrm{C}(13)-\mathrm{H}(13 \mathrm{C})$ & 109.5 \\
\hline
\end{tabular}




$\begin{array}{llll}\mathrm{H}(13 \mathrm{~A})-\mathrm{C}(13)-\mathrm{H}(13 \mathrm{C}) & 109.5 & \mathrm{H}(17 \mathrm{~A})-\mathrm{C}(17)-\mathrm{H}(17 \mathrm{~B}) & 109.5 \\ \mathrm{H}(13 \mathrm{~B})-\mathrm{C}(13)-\mathrm{H}(13 \mathrm{C}) & 109.5 & \mathrm{C}(15)-\mathrm{C}(17)-\mathrm{H}(17 \mathrm{C}) & 109.5 \\ \mathrm{C}(12)-\mathrm{C}(14)-\mathrm{H}(14 \mathrm{~A}) & 109.5 & \mathrm{H}(17 \mathrm{~A})-\mathrm{C}(17)-\mathrm{H}(17 \mathrm{C}) & 109.5 \\ \mathrm{C}(12)-\mathrm{C}(14)-\mathrm{H}(14 \mathrm{~B}) & 109.5 & \mathrm{H}(17 \mathrm{~B})-\mathrm{C}(17)-\mathrm{H}(17 \mathrm{C}) & 109.5 \\ \mathrm{H}(14 \mathrm{~A})-\mathrm{C}(14)-\mathrm{H}(14 \mathrm{~B}) & 109.5 & \mathrm{C}(20)-\mathrm{C}(18)-\mathrm{C}(19) & 111.8(2) \\ \mathrm{C}(12)-\mathrm{C}(14)-\mathrm{H}(14 \mathrm{C}) & 109.5 & \mathrm{C}(20)-\mathrm{C}(18)-\mathrm{P}(2) & 115.74(16) \\ \mathrm{H}(14 \mathrm{~A})-\mathrm{C}(14)-\mathrm{H}(14 \mathrm{C}) & 109.5 & \mathrm{C}(19)-\mathrm{C}(18)-\mathrm{P}(2) & 111.91(17) \\ \mathrm{H}(14 \mathrm{~B})-\mathrm{C}(14)-\mathrm{H}(14 \mathrm{C}) & 109.5 & \mathrm{C}(20)-\mathrm{C}(18)-\mathrm{H}(18) & 105.5 \\ \mathrm{C}(17)-\mathrm{C}(15)-\mathrm{C}(16) & 110.47(19) & \mathrm{C}(19)-\mathrm{C}(18)-\mathrm{H}(18) & 105.5 \\ \mathrm{C}(17)-\mathrm{C}(15)-\mathrm{P}(2) & 112.61(16) & \mathrm{P}(2)-\mathrm{C}(18)-\mathrm{H}(18) & 105.5 \\ \mathrm{C}(16)-\mathrm{C}(15)-\mathrm{P}(2) & 108.85(15) & \mathrm{C}(18)-\mathrm{C}(19)-\mathrm{H}(19 \mathrm{~A}) & 109.5 \\ \mathrm{C}(17)-\mathrm{C}(15)-\mathrm{H}(15) & 108.3 & \mathrm{C}(18)-\mathrm{C}(19)-\mathrm{H}(19 \mathrm{~B}) & 109.5 \\ \mathrm{C}(16)-\mathrm{C}(15)-\mathrm{H}(15) & 108.3 & \mathrm{H}(19 \mathrm{~A})-\mathrm{C}(19)-\mathrm{H}(19 \mathrm{~B}) & 109.5 \\ \mathrm{P}(2)-\mathrm{C}(15)-\mathrm{H}(15) & 108.3 & \mathrm{C}(18)-\mathrm{C}(19)-\mathrm{H}(19 \mathrm{C}) & 109.5 \\ \mathrm{C}(15)-\mathrm{C}(16)-\mathrm{H}(16 \mathrm{~A}) & 109.5 & \mathrm{H}(19 \mathrm{~A})-\mathrm{C}(19)-\mathrm{H}(19 \mathrm{C}) & 109.5 \\ \mathrm{C}(15)-\mathrm{C}(16)-\mathrm{H}(16 \mathrm{~B}) & 109.5 & \mathrm{H}(19 \mathrm{~B})-\mathrm{C}(19)-\mathrm{H}(19 \mathrm{C}) & 109.5 \\ \mathrm{H}(16 \mathrm{~A})-\mathrm{C}(16)-\mathrm{H}(16 \mathrm{~B}) & 109.5 & \mathrm{C}(18)-\mathrm{C}(20)-\mathrm{H}(20 \mathrm{~A}) & 109.5 \\ \mathrm{C}(15)-\mathrm{C}(16)-\mathrm{H}(16 \mathrm{C}) & 109.5 & \mathrm{C}(18)-\mathrm{C}(20)-\mathrm{H}(20 \mathrm{~B}) & 109.5 \\ \mathrm{H}(16 \mathrm{~A})-\mathrm{C}(16)-\mathrm{H}(16 \mathrm{C}) & 109.5 & \mathrm{H}(20 \mathrm{~A})-\mathrm{C}(20)-\mathrm{H}(20 \mathrm{~B}) & 109.5 \\ \mathrm{H}(16 \mathrm{~B})-\mathrm{C}(16)-\mathrm{H}(16 \mathrm{C}) & 109.5 & \mathrm{C}(18)-\mathrm{C}(20)-\mathrm{H}(20 \mathrm{C}) & 109.5 \\ \mathrm{C}(15)-\mathrm{C}(17)-\mathrm{H}(17 \mathrm{~A}) & 109.5 & \mathrm{H}(20 \mathrm{~A})-\mathrm{C}(20)-\mathrm{H}(20 \mathrm{C}) & 109.5 \\ \mathrm{C}(15)-\mathrm{C}(17)-\mathrm{H}(17 \mathrm{~B}) & 109.5 & \mathrm{H}(20 \mathrm{~B})-\mathrm{C}(20)-\mathrm{H}(20 \mathrm{C}) & 109.5 \\ & & & \\ & & & \end{array}$


Table S-24. Anisotropic displacement parameters $\left(\AA^{2} \times 10^{3}\right)$ for jonlm21 (2d). The anisotropic displacement factor exponent takes the form: $-2 \pi^{2}\left[h^{2} a^{* 2} U_{11}+\ldots+2 h k a^{*} b^{*} U_{12}\right]$

\begin{tabular}{|c|c|c|c|c|c|c|}
\hline & $\mathrm{U}_{11}$ & $\mathrm{U}_{22}$ & $\mathrm{U}_{33}$ & $\mathrm{U}_{23}$ & $\mathrm{U}_{13}$ & $\mathrm{U}_{12}$ \\
\hline W1 & $7(1)$ & $8(1)$ & $10(1)$ & $-1(1)$ & $0(1)$ & $-1(1)$ \\
\hline P1 & $9(1)$ & $9(1)$ & 11(1) & $-1(1)$ & $-1(1)$ & $0(1)$ \\
\hline P2 & $9(1)$ & $10(1)$ & $11(1)$ & $-1(1)$ & $0(1)$ & $0(1)$ \\
\hline $\mathrm{O} 1$ & $14(1)$ & $33(1)$ & $28(1)$ & $-10(1)$ & $0(1)$ & $-9(1)$ \\
\hline $\mathrm{O} 2$ & $34(1)$ & $26(1)$ & $15(1)$ & $1(1)$ & $-3(1)$ & $8(1)$ \\
\hline $\mathrm{O} 3$ & $27(1)$ & $24(1)$ & $17(1)$ & $8(1)$ & $4(1)$ & $2(1)$ \\
\hline $\mathrm{O} 4$ & $10(1)$ & $13(1)$ & $17(1)$ & $-4(1)$ & $-1(1)$ & $-1(1)$ \\
\hline O5 & $9(1)$ & $14(1)$ & $18(1)$ & $-1(1)$ & $-1(1)$ & $0(1)$ \\
\hline N1 & $8(1)$ & $10(1)$ & $10(1)$ & $-1(1)$ & $0(1)$ & $-1(1)$ \\
\hline $\mathrm{C} 1$ & $14(1)$ & $13(1)$ & $16(1)$ & $-5(1)$ & $0(1)$ & $-1(1)$ \\
\hline $\mathrm{C} 2$ & $13(1)$ & $13(1)$ & $16(1)$ & $-1(1)$ & $-1(1)$ & $2(1)$ \\
\hline $\mathrm{C} 3$ & $12(1)$ & 11(1) & $17(1)$ & $0(1)$ & $0(1)$ & $1(1)$ \\
\hline $\mathrm{C} 4$ & $10(1)$ & $13(1)$ & $10(1)$ & $1(1)$ & $-1(1)$ & $-2(1)$ \\
\hline C5 & $13(1)$ & $15(1)$ & $14(1)$ & $0(1)$ & $-2(1)$ & $-5(1)$ \\
\hline C6 & $10(1)$ & $21(1)$ & $16(1)$ & $2(1)$ & $-2(1)$ & $-5(1)$ \\
\hline C7 & $8(1)$ & $20(1)$ & $13(1)$ & $3(1)$ & $-1(1)$ & $-1(1)$ \\
\hline $\mathrm{C} 8$ & $9(1)$ & $13(1)$ & $9(1)$ & $3(1)$ & $-1(1)$ & $0(1)$ \\
\hline C9 & $19(1)$ & $12(1)$ & $14(1)$ & $-1(1)$ & $-2(1)$ & $2(1)$ \\
\hline C10 & $30(1)$ & $19(1)$ & $17(1)$ & $4(1)$ & $0(1)$ & $-5(1)$ \\
\hline C11 & $21(1)$ & $24(1)$ & $21(1)$ & $3(1)$ & $-7(1)$ & $6(1)$ \\
\hline C12 & $14(1)$ & $15(1)$ & $12(1)$ & $-3(1)$ & $1(1)$ & $0(1)$ \\
\hline C13 & $15(1)$ & $39(2)$ & $24(1)$ & $-10(1)$ & $6(1)$ & $-4(1)$ \\
\hline C14 & $40(2)$ & $21(1)$ & $22(1)$ & $-11(1)$ & $9(1)$ & $-5(1)$ \\
\hline C15 & $13(1)$ & $15(1)$ & $14(1)$ & $-3(1)$ & $1(1)$ & $4(1)$ \\
\hline C16 & $18(1)$ & $25(1)$ & $22(1)$ & $-9(1)$ & $-2(1)$ & $-3(1)$ \\
\hline C17 & $21(1)$ & $24(1)$ & $14(1)$ & $0(1)$ & $4(1)$ & $4(1)$ \\
\hline C18 & $19(1)$ & $13(1)$ & $14(1)$ & $3(1)$ & $1(1)$ & $2(1)$ \\
\hline C19 & $26(1)$ & $17(1)$ & $27(1)$ & $4(1)$ & $5(1)$ & $-3(1)$ \\
\hline $\mathrm{C} 20$ & $26(1)$ & $21(1)$ & $27(1)$ & $7(1)$ & $2(1)$ & $11(1)$ \\
\hline
\end{tabular}


Table S-25. Hydrogen coordinates (x 104) and isotropic displacement parameters $\left(\AA^{2} \times 10^{3}\right)$ for jonlm21(2d).

\begin{tabular}{|c|c|c|c|c|}
\hline & $\mathrm{x}$ & $\mathrm{y}$ & $\mathrm{z}$ & $\mathrm{U}(\mathrm{eq})$ \\
\hline H5 & -1002 & 1583 & 8289 & 17 \\
\hline H6 & -2763 & 2726 & 8519 & 19 \\
\hline H7 & -2550 & 4723 & 8817 & 17 \\
\hline H9 & 3432 & 905 & 8789 & 18 \\
\hline $\mathrm{H} 10 \mathrm{~A}$ & 2488 & 630 & 9881 & 33 \\
\hline H10B & 1465 & 979 & 9318 & 33 \\
\hline $\mathrm{H} 10 \mathrm{C}$ & 1951 & 1948 & 9854 & 33 \\
\hline H11A & 4655 & 1369 & 9783 & 33 \\
\hline H11B & 4224 & 2718 & 9726 & 33 \\
\hline $\mathrm{H} 11 \mathrm{C}$ & 5070 & 2146 & 9135 & 33 \\
\hline H12 & 3017 & 3057 & 7326 & 17 \\
\hline H13A & 5116 & 2903 & 7202 & 39 \\
\hline H13B & 5273 & 2281 & 7937 & 39 \\
\hline $\mathrm{H} 13 \mathrm{C}$ & 4886 & 3644 & 7892 & 39 \\
\hline H14A & 3552 & 1226 & 6908 & 41 \\
\hline H14B & 2366 & 1057 & 7396 & 41 \\
\hline $\mathrm{H} 14 \mathrm{C}$ & 3723 & 705 & 7668 & 41 \\
\hline H15 & 177 & 7848 & 9859 & 17 \\
\hline H16A & 1923 & 8020 & 10607 & 33 \\
\hline H16B & 2339 & 8188 & 9822 & 33 \\
\hline $\mathrm{H} 16 \mathrm{C}$ & 2601 & 6964 & 10207 & 33 \\
\hline H17A & -21 & 6797 & 10878 & 29 \\
\hline H17B & 791 & 5724 & 10585 & 29 \\
\hline $\mathrm{H} 17 \mathrm{C}$ & -570 & 5972 & 10282 & 29 \\
\hline H18 & 659 & 7193 & 7984 & 19 \\
\hline H19A & 1911 & 8833 & 7946 & 35 \\
\hline H19B & 2684 & 7788 & 8300 & 35 \\
\hline $\mathrm{H} 19 \mathrm{C}$ & 2100 & 8818 & 8763 & 35 \\
\hline $\mathrm{H} 20 \mathrm{~A}$ & -454 & 8893 & 8103 & 37 \\
\hline H20B & -252 & 8907 & 8920 & 37 \\
\hline $\mathrm{H} 20 \mathrm{C}$ & -1105 & 7911 & 8574 & 37 \\
\hline
\end{tabular}


Table S-26. Torsion angles $\left[{ }^{\circ}\right]$ for jonlm21(2d).

\begin{tabular}{|c|c|c|c|}
\hline C12-P1-O4-C4 & $-132.85(16)$ & $\mathrm{O} 4-\mathrm{P} 1-\mathrm{C} 9-\mathrm{C} 10$ & $-43.88(17)$ \\
\hline C9-P1-O4-C4 & $119.70(16)$ & C12-P1-C9-C10 & $-144.77(16)$ \\
\hline W1-P1-O4-C4 & $-9.11(16)$ & W1-P1-C9-C10 & $69.46(18)$ \\
\hline C18-P2-O5-C8 & $119.89(15)$ & O4-P1-C9-C11 & $-166.87(16)$ \\
\hline C15-P2-O5-C8 & $-133.04(15)$ & C12-P1-C9-C11 & $92.24(17)$ \\
\hline W1-P2-O5-C8 & $-3.58(15)$ & W1-P1-C9-C11 & $-53.54(19)$ \\
\hline P1-O4-C4-N1 & $11.4(3)$ & O4-P1-C12-C13 & $-178.06(18)$ \\
\hline $\mathrm{P} 1-\mathrm{O} 4-\mathrm{C} 4-\mathrm{C} 5$ & $-169.19(15)$ & C9-P1-C12-C13 & $-76.2(2)$ \\
\hline C8-N1-C4-O4 & 176.22(17) & W1-P1-C12-C13 & $71.3(2)$ \\
\hline W1-N1-C4-O4 & $-7.4(2)$ & O4-P1-C12-C14 & $-50.2(2)$ \\
\hline C8-N1-C4-C5 & $-3.2(3)$ & C9-P1-C12-C14 & $51.6(2)$ \\
\hline W1-N1-C4-C5 & $173.14(15)$ & W1-P1-C12-C14 & $-160.86(16)$ \\
\hline $\mathrm{O} 4-\mathrm{C} 4-\mathrm{C} 5-\mathrm{C} 6$ & $-176.95(19)$ & O5-P2-C15-C17 & $49.62(17)$ \\
\hline N1-C4-C5-C6 & $2.5(3)$ & $\mathrm{C} 18-\mathrm{P} 2-\mathrm{C} 15-\mathrm{C} 17$ & $149.86(16)$ \\
\hline C4-C5-C6-C7 & $0.1(3)$ & W1-P2-C15-C17 & $-64.05(18)$ \\
\hline C5-C6-C7-C8 & $-1.8(3)$ & O5-P2-C15-C16 & $172.47(16)$ \\
\hline C4-N1-C8-O5 & $-179.06(17)$ & $\mathrm{C} 18-\mathrm{P} 2-\mathrm{C} 15-\mathrm{C} 16$ & $-87.29(18)$ \\
\hline W1-N1-C8-O5 & $4.6(2)$ & W1-P2-C15-C16 & $58.80(19)$ \\
\hline C4-N1-C8-C7 & $1.4(3)$ & O5-P2-C18-C20 & $52.2(2)$ \\
\hline W1-N1-C8-C7 & $-174.91(14)$ & $\mathrm{C} 15-\mathrm{P} 2-\mathrm{C} 18-\mathrm{C} 20$ & $-49.5(2)$ \\
\hline $\mathrm{P} 2-\mathrm{O} 5-\mathrm{C} 8-\mathrm{N} 1$ & $-0.1(2)$ & W1-P2-C18-C20 & $162.40(16)$ \\
\hline P2-O5-C8-C7 & $179.43(14)$ & O5-P2-C18-C19 & $-178.11(17)$ \\
\hline C6-C7-C8-N1 & $1.1(3)$ & C15-P2-C18-C19 & $80.15(18)$ \\
\hline C6-C7-C8-O5 & $-178.49(18)$ & W1-P2-C18-C19 & $-67.90(18)$ \\
\hline
\end{tabular}

